\title{
DIMITRIS PARASCHAKIS
} ALGORITHMIC AND ETHICAL ASPECTS OF RECOMMENDER SYSTEMS IN E-COMMERCE

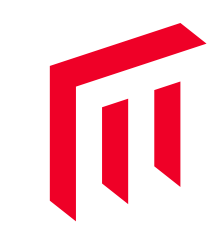



ALGORITHMIC AND ETHICAL ASPECTS OF RECOMMENDER SYSTEMS IN E-COMMERCE 


\section{Malmö University}

Studies In Computer Science No 4, Licentiate Thesis

(C) Dimitris Paraschakis, 2018

ISBN 978-91-7104-900-1 (print)

ISBN 978-91-7104-901-8 (pdf)

Holmbergs, Malmö 2018 


\section{DIMITRIS PARASCHAKIS}

ALGORITHMIC AND ETHICAL ASPECTS OF RECOMMENDER SYSTEMS IN E-COMMERCE

Department of Computer Science

Faculty of Technology and Society Malmö University, 2018 


\section{Studies in Computer Science}

Faculty of Technology and Society Malmö University

1. Jevinger, Åse. Toward intelligent goods: characteristics, architectures and applications, 2014, Doctoral dissertation.

2. Dahlskog, Steve. Patterns and procedural content generation in digital games: automatic level generation for digital games using game design patterns, 2016, Doctoral dissertation.

3. Fabijan, Aleksander. Developing the right features: the role and impact of customer and product data in software product development, 2016, Licentiate thesis.

4. Paraschakis, Dimitris. Algorithmic and ethical aspects of recommender systems in e-commerce, 2018, Licentiate thesis.

Electronically available at:

https://muep.mau.se/handle/2043/24268 
In memory of a beautiful soul 



\section{ABSTRACT}

Recommender systems have become an integral part of virtually every e-commerce application on the web. The deployment of these expert systems has enabled users to quickly discover the products or services they need, at the same time increasing business revenues through better customer conversion. Remaining a very active research field since the mid-2000s, recommender systems have been modeled using a plethora of machine learning techniques. However, the adoptability of these models by industrial e-commerce platforms remains unclear. In this thesis, we assess the receptiveness of industrial platforms to algorithmic contributions of the research community by surveying more than 30 popular shopping cart solutions, and experimenting with various recommendation algorithms on proprietary e-commerce datasets.

Another overlooked but important factor that complicates the design and use of recommender systems is their ethical implications. We provide a holistic view of these issues and summarize them in our ethical recommendation framework. This framework suggests new paradigm of ethics-awareness by design, and enables users to control sensitive moral aspects of recommendations via the proposed "ethical toolbox". The feasibility of this tool is supported by the results of our user study.

Since the large part of moral implications stems from user profiling, we investigate algorithms capable of generating useragnostic recommendations based solely on a visited product page. We propose an ensemble learning scheme based on Thompson Sampling bandit policy, which models arms as base recommendation functions. We show how to adapt this algorithm to realistic situations when neither arm availability nor reward stationarity is guaranteed. 


\section{PUBLICATIONS}

\section{Included conference papers}

- Comparative Evaluation of Top- $N$ Recommenders in Ecommerce: an Industrial Perspective [152] In proceedings of the 14th International Conference on Machine Learning and Applications (ICMLA), IEEE, 2015

- Ensemble Recommendations via Thompson Sampling: an Experimental Study within E-commerce [31] In proceedings of the 23rd International Conference on Intelligent User Interfaces (IUI), ACM, 2018

- Towards an Ethical Recommendation Framework [151] ${ }^{1}$ In proceedings of the 11th International Conference on Research Challenges in Information Science (RCIS), IEEE, 2017

\section{Related publications that are not included in the thesis}

- Recommender Systems from an Industrial and Ethical Perspective [150]

In proceedings of the 10th International Conference on Recommender Systems (RecSys), ACM, 2016

- Bandit Algorithms for e-Commerce Recommender Systems: Extended Abstract [29] In proceedings of the 11th International Conference on Recommender Systems (RecSys), ACM, 2017

- An Ensemble Recommender System for e-Commerce [30] In proceedings of the 26th Benelux Conference on Machine Learning (Benelearn), 2017

\footnotetext{
${ }^{1}$ Best paper award
} 
Personal contribution

In all included papers, the author of the thesis was the main contributor with respect to research planning, execution, and reporting. 


\section{ACKNOWLEDGMENTS}

I would like to express my gratitude to my supervisor Bengt J. Nilsson for his valuable advice and feedback. I am also grateful to all present and past members of the follow-up group for tracking my progress and reviewing the individual study plan: Jan Persson, Paul Davidsson, Mikael Hammar, Yuanji Cheng, Helena Holmström Olsson, Christina Bjerkén, Per Jönsson, Alexei Iantchenko.

Finally, I want to thank our industry collaborators Mikael Hammar and Björn Brodén from Apptus Technologies for the provided access to their datasets and development platform. Our frequent meetings with Apptus' research team have improved my understanding of how recommender systems are being approached in industry. 


\section{TABLE OF CONTENTS}

I COMPREHENSIVE SUMMARY 1

INTRODUCTION 2

Preamble..................... 2

From academia to industry . . . . . . . . . . 3

From algorithms to ethics . . . . . . . . . . . 7

Research questions .............. 10

$\begin{array}{ll}\text { BACKGROUND } & 12\end{array}$

Important challenges ............. 12

Problem formulation . . . . . . . . . . . . . . 14

Data representation ............... . . 15

Types of feedback . . . . . . . . . . . . . . 16

Evaluation methods . . . . . . . . . . . . . 18

Batch mode ............... . 18

Sequential mode .............. . 19

A/B testing ................ . . 20

Evaluation criteria . . . . . . . . . . . 21

Evaluation metrics . . . . . . . . . . . 22

Content-based filtering ... . . . . . . . . 24

Collaborative filtering ............ . . 26

K-Nearest Neighbors . . . . . . . . . . . . . 26

Association Rules Mining . . . . . . . . . . . . . 28

Matrix Factorization . . . . . . . . . . . . . 30

Multi-arm bandits . . . . . . . . . . . . . 32

Hybrid filtering ............. . . . . . . 34

Ethics in recommender systems . . . . . . . . . . . 35

Law, ethics, and morality . . . . . . . . . . . . . . . 35

Recommenders as moral agents . . . . . . . . . . 36

Moral responsibility . . . . . . . . . . . . . . 37

Principles of accountable algorithms . . . . . . . 38 
METHODOLOGY 39

Collaboration with Apptus Technologies . . . . . . . . . . 39

Choice of methods . . . . . . . . . . . . . . . . 40

Experiment . . . . . . . . . . . . . . . . . . 4 41

Design science . . . . . . . . . . . . . . . . . . . . 42

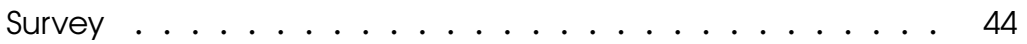

Literature review . . . . . . . . . . . . . . . . . . 45

Limitations . . . . . . . . . . . . . . . . . . . . . . . 47

PAPER OVERVIEW 49

Paper I: Comparative Evaluation of Top- $N$ Recommenders in

E-commmerce: an Industrial Perspective . . . . . . . . 50

Paper II: Ensemble Recommendations via Thompson Sampling:

an Experimental Study within E-commerce . . . . . . . 51

Paper III: Towards an Ethical Recommendation Framework . . 52

CONTRIBUTIONS 53

Research question $1 \ldots \ldots . \ldots . \ldots 53$

Research question $2 \ldots \ldots \ldots$. . . . . . . . . . . . . . . . . . . . . . .

Research question $3 \ldots \ldots$. . . . . . . . . . . . . . 59

CONCLUSIONS 62

Thesis summary . . . . . . . . . . . . . . . . . . . . . . 62

Future work . . . . . . . . . . . . . . . . . . . 64

\|I INCLUDED PAPERS

PAPER I 66

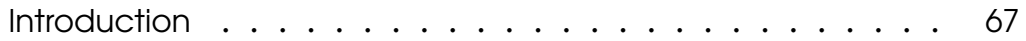

Related work . . . . . . . . . . . . . . . . . . 69

Methodology . . . . . . . . . . . . . . . 70

Datasets . . . . . . . . . . . . . . . . 71

Experimental protocol . . . . . . . . . . . . . . . 72

Evaluation metrics . . . . . . . . . . . . . . . . 74

Hyperparameter optimization via Golden Section Search . . . 76

Survey of deployed recommender systems . . . . . . . . . . 78 
Recommendation algorithms . . . . . . . . . . . . 78

MF-based methods . . . . . . . . . . . . . . . . . . . . 78

Data mining (association rules) . . . . . . . . . . . 80

Memory-based CF (K-nearest neighbors) . . . . . . . . 81

Results . . . . . . . . . . . . . . . . 82

Experiment 1. Non-chronological split . . . . . . . . . . 82

Experiment 2. Chronological split . . . . . . . . . . . . 84

Survey responses . . . . . . . . . . . . . . . . . . 88

Conclusions . . . . . . . . . . . . . . . . 90

Acknowledgment .................. . 91

PAPER II 92

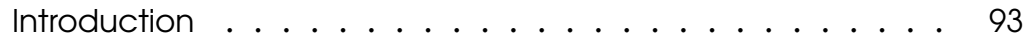

Related Work . . . . . . . . . . . . . . . . . . . . . . 95

Approach . . . . . . . . . . . . . . . . . . 98

Problem Setting . . . . . . . . . . . . . . . . . . . . . . . . . 9 98

Base Recommenders . . . . . . . . . . . . . . . . . . 100

Ensemble Learning Agent . . . . . . . . . . . . . 103

Experiments . . . . . . . . . . . . . . . . . 108

Datasets and Evaluation Metrics . . . . . . . . . . . 108

Experiment 1: Standard vs. Modified TS . . . . . . . . 109

Experiment 2: BEER(TS) vs. Baselines . . . . . . . . . 110

Experiment 3: MAB Policies within BEER . . . . . . . . . 112

Experiment 4: Priming the Sampler . . . . . . . . . . . . 112

Conclusions . . . . . . . . . . . . . . . 116

Contribution Summary . . . . . . . . . . . . 116

Future Work . . . . . . . . . . . . . . . . . . . 118

$\begin{array}{ll}\text { PAPER III } & 119\end{array}$

Introduction . . . . . . . . . . . . . 120

Theoretical background: ethical challenges . . . . . . . 121

Data collection and filtering . . . . . . . . . . . 121

Data publishing and anonymization . . . . . . . . . 125

Algorithmic opacity, biases, and behavior manipulation 129

Online experiments . . . . . . . . . . . . . . . . . . 133 
Summary as a framework . . . . . . . . . . 135

Feasibility Study . . . . . . . . . . . . . . . 137

Conclusion . . . . . . . . . . . . . . . 144

$\begin{array}{ll}\text { REFERENCES } & 146\end{array}$ 


\section{LIST OF FIGURES}

1 Session data . . . . . . . . . . . . . . . 16

2 Purchase matrix . . . . . . . . . . . . 16

3 Contingency table . . . . . . . . . . . . . . . 23

4 Characteristics of industrial recommendation engines (paper I) . . . . . . . . . . . . . . . . . 54

5 Timespan of datasets . . . . . . . . . . . . . . . 72

6 Distribution of purchases per customers . . . . . . . . 72

7 Representation of a temporal dataset. Horizontal lines represent customer profiles on a timeline, where purchases are marked with blue bars. . . . . . . . . . . . . . . 74

8 Chronological split of D1 with 9 training sets . . . . . . 85

9 Chronological split of D2 with 6 training sets . . . . . . . 86

10 Survey responses . . . . . . . . . . . . . . . . . . . 89

11 i2i session-based recommendations with explainable actions . . . . . . . . . . . . . . . . 100

12 Moral bonds between stakeholders in the data publishing process . . . . . . . . . . . . . . . . . . 128

13 High-level view of a personalized recommender system . 129 


\section{LIST OF TABLES}

1 Research methods . . . . . . . . . . . . . . . . . . 40

$2 \quad F 1 @ 5$ scores of different algorithms run on two e-commerce datasets (paper I) . . . . . . . . . . . . . . 55

3 Standard vs. modified Thompson Sampling (paper II) . . 58

4 Ethical recommendation framework (paper III) . . . . . 61

5 Summary for datasets D1 and D2 f . . . . . . . . 71

6 Scores for the random split on D1 . . . . . . . . . 83

7 Scores for the random split on D2 . . . . . . . . . . 83

8 Average scores for the chronological split on D1 . . . . . 87

9 Average scores for the chronological split on D2 . . . . 87

10 Dataset summary . . . . . . . . . . . . . . . . . . . . 108

11 Standard vs. modified Thompson Sampling . . . . . . . 110

12 Ensemble recommender vs. stand-alone baselines . . . 111

13 Ensemble learner with different MAB policies . . . . . . . 113

14 Priming the sampler with pre-recorded event data . . . 114

15 Catalog-based priming . . . . . . . . . . . . . 116

16 General user-centric ethical recommendation framework 136

17 Issue 1/5: User profiling . . . . . . . . . . . . . . . 139

18 Issue 2/5: Data sharing . . . . . . . . . . . . . . . . 140

19 Issue 3/5: Online experiments . . . . . . . . . . . . 141

20 Issue 4/5: Marketing bias . . . . . . . . . . . . . . . . 142

21 Issue 5/5: Content censorship . . . . . . . . . . . . . 142 


\section{LIST OF ALGORITHMS}

1 Recommendations in batch mode . . . . . . . . . . . 19

2 Recommendations in sequential mode . . . . . . . . 20

3 User-user KNN . . . . . . . . . . . . . . . . . . . . . . 27

4 Item-item KNN . . . . . . . . . . . . . . . . 27

5 Association rules mining . . . . . . . . . . . . . 29

6 Weighted regularized MF recommendations . . . . . . 31

7 Multi-arm bandit . . . . . . . . . . . . . . . . . 33

8 Golden Section Search . . . . . . . . . . . . . . . . 77

9 IDF-based partitioning . . . . . . . . . . . . . 103

10 BEER(TS) . . . . . . . . . . . . . . . 107 

PART I

COMPREHENSIVE SUMMARY 


\section{INTRODUCTION}

\section{Preamble}

The Web, they say, is leaving the era of search and entering one of discovery. What's the difference? Search is what you do when you're looking for something. Discovery is when something wonderful that you didn't know existed, or didn't know how to ask for, finds you.

- CNN Money, “The race to create a 'smart' Google”

Recommender systems (RS) have emerged as a sub-field of machine learning in the mid-1990s to address the important problem of choice overload, which has proven detrimental to our emotional and psychological well-being [183]. This has become especially prominent in the modern age of Big Data, where the smart discovery of relevant products or services is, at the very least, as important as search. The ability of an online service to provide user-tailored recommendations is a crucial factor for ensuring customer satisfaction and retention. Nowadays, the application of recommender systems spans numerous domains, including streaming media (Netflix, Spotify), news (CNN, Google News), dating (Tinder, Libimseti.cz), food (Food.com, Epicurious), travel (Booking.com, AirBnB), social networks (Xing, LinkedIn), and many others. However, one of the oldest and largest domains with a major need for recommender systems is, of course, e-commerce. The figures estimated for the world's largest retailer - Amazon - are astonishing: there are more than 300 million registered users and more than a billion listed products worldwide $[72,1]$. Mining relevant products from this massive catalog for so many users is a very challenging task. However, it is also a highly rewarding one: reportedly, 35\% of sales on Amazon result from recommendations [127]. Therefore, a recommender system hits two targets at the same time: (1) to help users quickly discover the products they need; and (2) to 
help businesses increase their revenues. Keeping user satisfaction and economic incentives in balance necessitates strict adherence to ethical principles on every stage of RS development and use. In this thesis, we investigate both the algorithmic and the moral aspects of recommender systems in e-commerce.

\section{From academia to industry}

Academia solves simple problems with complex methods. Industry solves complex problems with simple methods.

- Björn Brodén, Apptus Technologies

In spite of that recommender systems have been known since early 1990s, the academic interest toward this field has dramatically increased after the announcement of the Netflix Prize ${ }^{2}$ contest in 2006. With a million dollars prize money, Netflix went on a quest for recommendation algorithms capable of surpassing the accuracy of Netflix's own machinery by $10 \%$. This contest spurred active research within the field, yielding a variety of recommendation techniques over the past decade. Surprisingly, the prize-winning algorithm was never put to real use: Netflix concluded that the measured accuracy gains "did not seem to justify the engineering effort needed to bring them into a production environment" [8]. This interesting fact calls for a deeper analysis of the gap between research contributions and commercial applications, which has rarely been discussed in the RS literature. In the context of this thesis, it would be interesting to investigate how receptive the e-commerce domain is to the recent algorithmic advances in the field of recommender systems. This analysis must reveal which algorithmic approaches and which evaluation strategies are most appropriate for modeling recommender systems in this domain.

The first notable observation is that the Netflix problem was essentially formulated as a rating prediction task. This has the following rationale: if we can accurately predict user's ratings

\footnotetext{
${ }^{2}$ http://www.netflixprize.com/
} 
for unseen movies, then we can pick the most highly scored ones among these movies as our recommendations to that user. Due to this problem formulation, and because of the early availability of datasets containing movie and music ratings, the majority of recommender systems proposed by researchers have been modeled as rating predictors and evaluated as such. In contrast, e-commerce is characterized by the general lack of ratings and has to rely on other types of information for generating recommendations, such as click and purchase data. This data has a binary representation and hence requires different algorithmic approaches, as we move from rating prediction to the so-called "top- $N$ ranking" problem. This approach to recommendations is somewhat less understood and is an active area of research.

Whichever the approach is, building accurate models has clearly been the main focus of academic endeavors within the RS field. From the industry perspective, creating an accurate predictor is not everything that is required to build a good recommendation engine [5]. Some of the crucial factors that determine the commercial viability of a given method include its ability to scale with growing numbers of users/items, and to compute recommendations in a fraction of a second. Notably, only $11 \%$ of RS publications report runtimes [19]. Therefore, the suitability of many elaborate recommendation techniques to the realities of the industrial sector remains unclear.

Another important aspect concerns not the algorithms themselves, but their evaluation methods. Since in many cases researchers are deprived the ability to test their recommender systems online, they use offline simulations to reason about the model's performance. Companies also run such simulations to determine whether an algorithm is to be further "A/B tested" on real users. Consequently, offline evaluation protocols should strive to approximate the online settings as closely as possible. The traditional methodology of offline testing practiced by RS researchers presupposes the existence of a large chunk of 
recorded data, which is split into training and test sets by randomly withholding certain percentage of ratings from each customer profile. The main issue with this approach is that it does not respect the sequence of events, resulting in accuracy estimates that may not correlate well with the real world. Whereas the exact time of rating a movie might not play a crucial role in modeling user preferences in the movie domain, knowing which click was followed by which purchase is of high value to an e-commerce recommender system. In paper I, we report our experiments on random and chronological splits of several purchase datasets. Regardless of how splitting is done, this batch evaluation method limits itself to one particular state of the environment determined by the split point. A much more realistic way of simulating the real recommendation process is to evaluate the system at each time step in a sequential manner. In this setting, after each newly issued recommendation event, the system gathers feedback in the form of "rewards" and updates itself according to how the user reacts on the recommendation. The final score is calculated as the total reward over all time steps. This scenario is a typical instance of reinforcement learning. It also closely resembles online shopping sessions in e-commerce, where the system is rewarded for recommending products that are eventually purchased at the end of the session. Surprisingly, the sequential protocol has been left aside in the RS literature [74].

Furthermore, the adoption of the sequential learning in academic research would invalidate most recommendation techniques that perform well in batch mode. There are several reasons for that. First, this protocol dictates incremental training, which is not supported by many algorithms out-of-the-box. Second, in the sequential setting the system is evaluated from "time zero", meaning that it should be able to produce recommendations under cold-start. However, discarding customers with less than $x$ number of ratings from the evaluation procedure is still a common practice among RS researchers. Lastly, 
the system must be able to compute recommendations very fast in real time. Consequently, many known recommendations techniques will need substantial adjustments to accommodate the above requirements.

Coming back to the Netflix Prize contest, it is worth noting that the prize was won by an ensemble learning method, proving it a very promising strategy. The idea behind ensemble learning is to combine the output of several "weak learners" so that they reinforce each other in an optimal way. Some of the well-known ensemble methods are bagging [28], boosting [181], and stacking [209]. The interesting question is, how can we build an ensemble recommender system that would perform well in sequential mode that we advocated earlier? How do we make it suitable for the deployment on commercial platforms? The reinforcement learning paradigm suggests that such a system could be modeled as a multi-arm bandit problem. Assuming that we already have a collection of basic recommendation components, we can represent them as bandit arms. The selection of an arm at each time step is then decided by some bandit policy. Adapting this approach to real-world scenarios would require constructing simple but fast recommendation components, and adapting the bandit policy to realistic situations when these components cannot produce recommendations for certain queries, or when they do not have a fixed probability of generating a reward. Investigating this direction is one of the main goals of this thesis. 


\section{From algorithms to ethics}

There is no right way to do a wrong thing.

- Harold S. Kushner, author

Whenever algorithms get involved in decision making, the issues of ethics inevitably come to the forefront. Recommender systems are no exception. Yet, the discussion around recommendation ethics is virtually non-existent in the literature. According to the recent study by Tang \& Winoto [193], there exist only three publications (namely, [169, 186, 193]) that specifically address ethics-aware recommendations. Still, these and similar works (e.g. [83]) focus on specific moral aspects in specific recommendation scenarios. In our opinion, the holistic view on the ethics of recommendations is currently lacking in this field. Knowing what ethical issues may appear on each stage of RS development can hopefully contribute to the emergence of more user-friendly, privacy-preserving, and nondiscriminatory recommendation algorithms. Investigating existing examples of ethical problems scarcely reported in the RS literature would help to frame a coherent classification of these ethical issues and their potential solutions. One of such motivating examples brings us back to the Netflix Prize. Two years after its public release, the Netflix dataset was de-anonymized via a linking attack [139], putting a privacy of its 500,000 uses at risk. This resulted in a lawsuit and put an end to the planned Netflix Prize sequel [107] due to the raised privacy concerns. Clearly, personal privacy is one of the biggest issues in the ethical discourse around the practices of Big Data analytics, which has to do with the leakage of confidential personal information contained in user profiles. The "item-to-item" recommendation scenario that we analyze in [31] does not make assumptions about the existence of user profiles. In this scenario, recommendations are based solely on the page visits within an anonymous shopping session. This makes the system immune to privacy threats that personalized services are prone to. The 
privacy implications have been thoroughly studied by Friedman et al. [65] in the context of recommender systems. To derive a classification of recommendation ethics, it would help to study other moral issues beyond privacy and anonymization in the same context.

The three biggest challenges of today's Web, according to its creator Tim Berners-Lee, are (1) the loss of control over personal data, (2) the easy spread of misinformation, and (3) the lack of transparency [60]. We can see that all these are ethicsrelated issues, since they pose a threat to the general moral principle of "do no harm". It is not hard to find examples in the RS literature that relate to each of those issues. For instance, the Netflix case mentioned earlier exemplifies the "failure of anonymization" [147], which is responsible for issue (1). Issue (2) appears particularly relevant to recommender systems in the light of the recent controversy around the election of Donald Trump, which has been largely attributed to the dissemination of fake stories via news recommenders on social media. Finally, the lack of transparency mentioned in issue (3) makes it possible to hide the evidence of algorithmic discrimination, leading to such known incidents as recommendations of lower paying jobs to female candidates or higher priced flights to MacBook owners [168].

Koene et al. [107] point out that today's research practice in personalization and recommender systems is dangerously unbalanced, as it puts commercial success above considerations of the moral impact of this technology. The authors see it as a worrying sign that may eventually lead to the public backlash against this research area, similar to what happened to the Bioscience field in the 1990s because of the GMO crop controversy. Fortunately, the FATML ${ }^{3}$ movement standing for Fairness, Accountability, and Transparency in Machine Learning has spurred active research on these topics in recent years.

\footnotetext{
${ }^{3}$ https : //www. fatml.org/
} 
FATML has formulated a set of five principles for determining the social and moral impact of algorithmic decision making: responsibility, explainability, accuracy, auditability, and fairness. Their purpose is to "help developers design and implement algorithmic systems in publicly accountable ways" [53]. We briefly overview these principles in section Principles of accountable algorithms. With the research presented in this thesis, we hope to draw attention to these topics in the narrower field of RS, which clearly represents a class of such algorithmic systems.

Many ethical issues in machine learning are addressed by either technological or normative means (or both). Normative solutions are implemented via regulatory incentives, such as the White House's Consumer Privacy Bill of Rights ${ }^{4}$, or the EU's Data Protection Directive ${ }^{5}$. As these solutions fall on the legal side of ethics, we leave them outside the scope of this thesis. Besides, the contractarian ethical theory, wherein morality is bounded by legal and social contracts, has been criticized for its serious limitations [194]. As an example, consider a system that recommends the same product at different prices to different customers. Given that such practice is perfectly legal in some jurisdictions, would it also be morally acceptable? Indeed, when such an experiment was attempted by Amazon, it provoked a furious reaction of the user community [200]. Finally, legislators struggle to keep up with the technological progress in the web sphere and the new threats that become evident long before policies can adapt to them.

We therefore advocate complex solutions, where existing policies are backed with special-purpose technology designed to protect the moral rights and liberties of RS users. At this stage, there seems to be a lack of consensus amongst corporate and

\footnotetext{
${ }^{4}$ https://obamawhitehouse.archives.gov/sites/default/ files/privacy-final.pdf

${ }^{5}$ http://eur-lex.europa.eu/legal-content/en/TXT/?uri= CELEX : 31995L0046
} 
academic researchers with respect to the "solutions tookit" [60] with exact methods for enabling algorithmic accountability. In this thesis, we propose our variant of an "ethical toolbox" for RS users as a means to provide them manual controls over the morally sensitive aspects of a recommender system. In our view, the provision of such tools promotes the paradigm of ethics-awareness by design, and can complement existing technological solutions, such as privacy-preserving data publishing / collaborative filtering, or discrimination-free data mining. The results of the feasibility study we report in [151] show promising prospects for the further research and development of such user-centric toolkits.

\section{Research questions}

This thesis addresses both the algorithmic and the ethical side of recommender systems. Even though these aspects are interrelated, it helps to break our research into distinct directions. For each of them, we formulate a general research question (RQ), accompanied with more specific sub-RQs:

RQ1. How to assess the receptiveness of the e-commerce domain to recent algorithmic advances in the field of recommender systems?

RQ1.1. Which recommendation algorithms are suitable for e-commerce settings, where explicit ratings are inexistent and implicit information is sparse?

RQ1.2. How can temporal information available in realworld datasets contribute to more realistic offline evaluation of recommender systems?

RQ2. How to build an ensemble of elementary recommendation components with non-stationary rewards and varied availability? 
RQ2.1. How does Thompson Sampling compare to other bandit policies for orchestrating the ensemble in sequential settings?

RQ2.2. How does priming the ensemble with prior knowledge affect its performance and how can such priming be done?

R63. What are the ethical challenges that complicate the design and use of recommender systems, and what are the possible solutions?

RQ3.1. How to aid morality in recommender systems through user engagement?

Outline The remainder of the thesis is organized as follows. In the next section, we provide the necessary background and build the intuition for understanding the rest of the thesis. After that, we detail our methodological approach with a separate discussion of each research method involved. Next, we briefly overview the included papers. We then go on with the discussion of our contributions to each of the research questions formulated above. The final section summarizes the thesis and draws avenues for future research. 


\section{BACKGROUND}

This section provides the selected background material on recommender systems that is necessary for understanding the content of included papers. We start by outlining important challenges of the e-commerce domain and define the recommendation problem. We then introduce different types of data representation and user feedback. Further, we explain how these systems are evaluated based on the user feedback they record. We then present the basic algorithmic ideas behind the two main recommendation approaches: content-based filtering and collaborative filtering. We also discuss their fusion in the form of hybrid and ensemble models. A multi-arm bandit paradigm is also introduced for better understanding the content of paper II. Finally, we give a gentle introduction into ethics and build the ground for more detailed discussion in paper III.

\section{Important challenges}

Here we outline some of the most important technical challenges of recommender systems in e-commerce, which became evident during our collaboration with Apptus Technologies.

Cold-start. As the user base and the product catalog are constantly evolving, the arrival of new items or users makes it difficult to use them in recommendations, since important historical data is missing. This is one of the most important challenges in recommender systems, known as the "cold-start" problem. In fact, e-commerce applications suffer from continuous cold-start, since users and items remain cold for long periods, and can even "cool down" after some time due to temporal volatility in user preferences or item utility [22]. 
Long tail effect. Most product catalogs exhibit the so-called "long tail" effect [77], where the "tail" contains the largest portion of the product catalog with items that have only few or zero purchases. Together, these purchases sum up to a significant total amount. Therefore, one of the main objectives of a recommender system is to boost the sales of such long-tail items, which is a harder task than recommending popular "head" items [14]. This effect is omnipresent in e-commerce.

Unidentified users. Recent e-marketplace reports indicate that more than a half of e-commerce sessions (57.1\%-62.3\%) are anonymous [196, 210]. This happens because users rarely register or log in during browsing, which of course impacts the ability to build long-term user profiles that are necessary for traditional recommendation techniques. This problem is addressed in session-based recommender systems that have begun to emerge in recent years (e.g. [87, 161, 210]). They strive to fully utilize all available information in current session to infer the intent of a user [160].

Lack of ratings. The vast majority of RS articles assume that businesses have access to rating data from customers [24]. For many e-commerce retailers, however, rating data is not available [158]. The main reasons for the absence of ratings is that users do not rate consumer products as often as they rate movies or music, and that obtaining quality ratings requires a proper survey mechanism to collect user feedback efficiently and accurately [24]. As a consequence, e-commerce platforms do not always support this functionality. This means that the majority of state-ofthe-art recommendation algorithms are not applicable to e-commerce data.

Alternative vs. complementary items. Unlike other domains, similarity-based recommendations in e-commerce are usu- 
ally less straightforward. For example, if a user has recently bought a laptop, recommending other computers to this customer would be a poor strategy. Instead, the system should be able to identify complementary products as potential recommendations for this user, in hope to generate an up-sale.

Seasonality effects. Many product categories in e-commerce have pronounced temporal relevance [180], which is not so common in more "traditional" RS domains such as movies. These categories include clothes, toys, holiday products, and other seasonal merchandise (sunscreens, snow shovels, etc.). The identification of such seasonal products can be done either manually or automatically, and has been shown to provide a notable accuracy gain [187].

We refer the reader to [167] for a wider discussion of existing and newly emerging challenges in recommender systems.

\section{Problem formulation}

As mentioned earlier, the recommendation task was initially formulated as a rating prediction problem. This corresponds to the following definition:

Definition 1. The task of a recommender system is to estimate the utility function that predicts how a user will like an item (i.e. preference).

In the absence of ratings, the definition becomes:

Definition 2. The task of a recommender system is to estimate the utility function that predicts whether the user will choose an item (i.e. relevance).

The predictions are essentially done on the basis of useritem or item-item associations. Defining and computing these associations is the key function of a recommender system. Once 
the utility function is known, the recommender system selects top- $N$ items sorted by the estimated utility score. A recommendation is considered successful when the suggested item is consumed by the user, where the definition of consumption is application-dependent (e.g. buying an item, watching a movie, listening to a song, etc.). On a higher level, the top- $N$ ranking itself is optimized for a particular goal (or a set of goals in multicriteria recommender systems). E-commerce recommenders, in particular, aim to optimize quantities such as revenues, sold units, click-through-rate, conversion rate, etc.

\section{Data representation}

E-commerce data are comprised of user sessions. A session is a sequence of user interactions (events) on a website, which starts as soon as the user enters the website, and ends when the user leaves the website or when a timeout has occurred. The most common events are clicks, searches, additions to shopping basket, and purchases. We can represent each event as a triplet $e=(a, i, t)$, where $a \in\{$ click, search, basket,purchase $\}$ is event type, $i \in I$ is an item from the product catalog $I$, and $t$ is the event's timestamp. A toy example of user sessions is depicted in Figure 1. In reality, user sessions do not always culminate in a purchase, and some users have multiple sessions (e.g. $S_{4}$ and $S_{5}$ may belong to the same user).

Notably, recommender systems operating on session data are still rather rare [87]. The common way of representing the recommendation problem is via a rating or a purchase matrix. Given the set of users $U$ and the set of items $I$, we define ma$\operatorname{trix} \mathbf{P}^{|U| \times|I|}$ with each entry $p_{u, i}$ storing the interaction of user $u \in U$ with item $i \in I$. Rows and columns of the matrix represent, respectively, user and item profiles. Figure 2 illustrates the purchase matrix with interactions taken from the example dataset of Figure 1, where each session is assumed to represent a distinct user. With this representation, the recommendation 


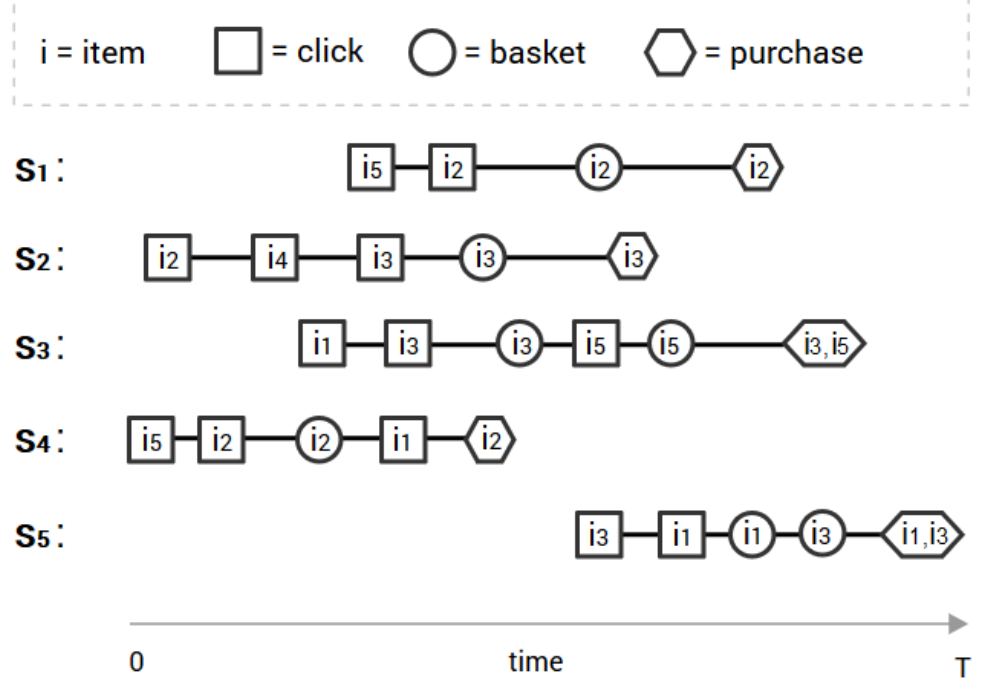

Figure 1: Session data

task boils down to a matrix completion problem. Sometimes, a higher dimensional tensor representation is used to incorporate additional contextual features. However, such context-aware systems will not be covered in this thesis.

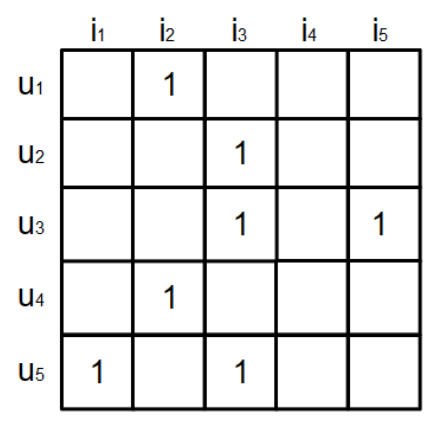

Figure 2: Purchase matrix

\section{Types of feedback}

Recommender systems rely on two types of user feedback: 
Explicit Feedback. This type of feedback is given directly by the user in the form of ratings. The most common example is when a user rates a watched movie on a scale from 1 to 5 . The system therefore receives an explicit preference score for a given user-item pair, based on which a ranking of items can be determined. Explicit preference acquisition makes this feedback unambiguous and reliable. However, there are a few drawbacks: (1) Rating data is typically sparse, as only a small fraction of consumed items are rated, (2) Rating functionality is not always supported by e-commerce platforms, (3) Ratingbased recommendations are made under the assumption that users are only interested in top-rated items. However, this does not always hold in practice. For example, cheaper items tend to have lower ratings than the expensive ones, but might nevertheless be preferred more often because of their price.

Implicit Feedback. This type of feedback is collected implicitly from various user interactions on a website, such as product page views, purchases, or additions to cart. The feedback that the system receives when such an event is registered as a result of successful recommendation takes the form of rewards. This implies binary preference, e.g. reward $=1$ if bought, reward $=0$ if not bought. The main advantage of this type of feedback is that it is generally denser and easier to collect than the explicit one, since no direct user input is required. However, this does not apply to all types of data. For instance, the typical goal of a recommender system on an e-commerce site is to predict future purchases that a user will make. Purchase events, however, are even sparser than rating data, since a user cannot rate an item until he/she buys it. Furthermore, implicit feedback is more ambiguous, since reward $=1$ does not convey whether the user liked the bought item, 
and reward $=0$ does not distinguish between items that were not examined, and items that were examined but not preferred. Therefore, it is generally less reliable than explicit feedback.

\section{Evaluation methods}

Due to our focus on e-commerce, we will cover evaluation metrics and algorithms for the top- $N$ recommendation problem and not those for rating prediction. The typical RS evaluation pipeline consists of an offline evaluation based on some prerecorded data, followed by an online evaluation on real users. This last phase is usually performed when there is sufficient evidence that the recommender outperforms some important baselines on recorded data. In academic research, online evaluation is difficult to perform and not generally practiced for evaluating proof-of-concept RS. The offline RS evaluation can be done in either batch or sequential (streaming) mode. We review each of these methods next.

\section{Batch mode}

The batch protocol remains by far the most widely used evaluation method in RS research. It takes a user-item matrix (like the one in Figure 2) as an input, and processes it in chunks, where the largest chunk is used for training the model, and the smaller, distinct chunk is kept for testing the accuracy of predictions. In some cases, a separate validation set is used for hyperparameter tuning. The division into training and test sets can be done in several ways:

Random split. This split can be done by shuffling events in user profiles and then moving a certain percentage of them to the test set. This amount usually ranges from 10\% to 50\%. The remaining events form the training set. By repeating this procedure $k$ times while ensuring that each event is seen only once in the test set, we arrive at the 
so-called $k$-fold cross-validation method. With increasing $k$ we increase the reliability of our estimates.

Chronological split. Splitting can also be done chronologically, when timestamps of events are available in the data. According to this strategy, the experimenter chooses a time instance, which divides user profiles into past and future events. The recommender is then trained on past data to predict future events contained in the test set. Needless to say, this technique handles streaming data better than the random strategy.

The recommender's operation in batch mode is summarized in Algorithm 1.

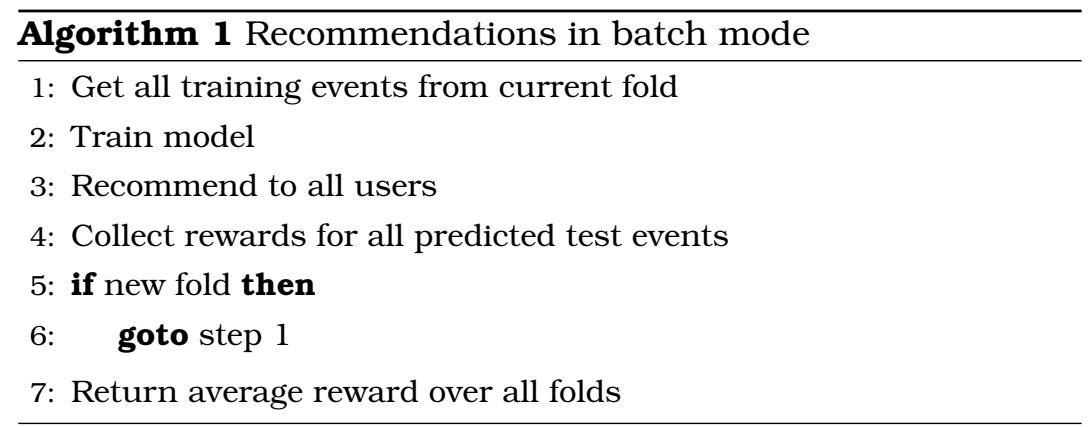

The batch operation is suitable for user-to-item recommendations, which rely on the estimation of similarities between a user profile and each candidate item of the product catalog.

Sequential mode

The sequential protocol more closely mimics the real recommendation process, and can be used directly on session data. It proceeds as shown in Algorithm 2.

A recommendation query is typically issued for each new user action in a session. For example, visiting the item's page triggers recommendations of alternative items from the same or another category (i.e. up- and cross-selling), the addition 


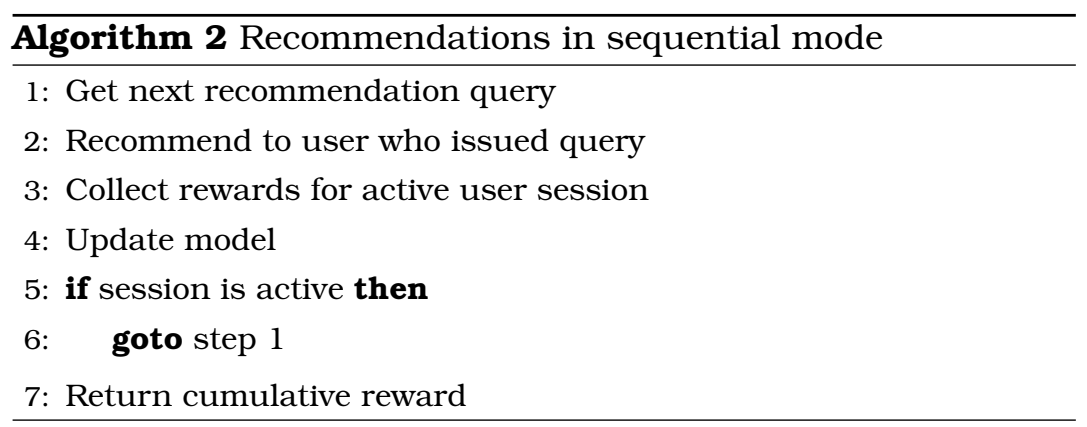

of an item to the shopping basket triggers recommendations of related item accessories, etc. Thus, the sequential protocol is well-suited for item-to-item recommendations, which are computed merely from their affinity to a single item. These types of recommendations exist on many major e-commerce platforms, such as iTunes store, Google Play and Amazon. Since such recommendations are given in the context of explicit user interest in a specific item, they often result in higher click-throughrate and greater share of sales and revenue [17], as compared to user-to-item recommendations that are common in batchoperated recommender systems.

Algorithm 2 instantiates a reinforcement learning scenario. This means that it requires different algorithmic approaches than the ones normally used for batch operation. We build the basic algorithmic intuition around both RS operation modes later in this section.

\section{A/B testing}

Unlike the previously discussed protocols, A/B testing is an online evaluation method, that is widely used for evaluating new algorithms, design features, or even entire web pages. It can be viewed as a two-sample hypothesis testing methodology, where the null hypothesis represents the control entity (" $A$ ") and the alternative hypothesis represents the treatment entity (" $B$ "). For a recommender system, the null hypothesis is 
associated with the current state of RS, while the alternative hypothesis typically represents a new algorithm, a modification of an existing algorithm, a new layout, etc. The user traffic is then randomly divided between $A$ and $B$, and the system is evaluated for some metric for a certain period of time. By measuring the difference between $A$ and $B$, it can be established whether or not the alternative hypothesis should be accepted. Compared to offline testing, A/B testing provides an evaluation setting that is much closer to a real recommender system [75]. Many big e-commerce businesses, such as Amazon and eBay, run online experiments on a continuous basis [159].

\section{Evaluation criteria}

Recommender systems can be assessed for a variety of criteria, which are covered in detail in [75]. Here we review some of these criteria that are particularly important for an e-commerce recommender system:

Accuracy. This is the key property that recommender systems are being assessed for. It can materialize in various measures depending on the goal of the application, such as purchase prediction, next click prediction, click-throughrate, conversion rate, or query abandonment.

Utility. In the e-commerce domain, maximizing purchases per se is not always the main objective. Instead, recommendations that serve some business-critical utility, such as revenues or customer retention, may be more important.

Coverage. A typical e-commerce product catalog suffers from a long-tail effect, which means that the largest part of the catalog is never consumed. Therefore, optimizing a recommender for a wider catalog coverage with the inclusion of long-tail items is often desirable. Likewise, the ability of generating accurate recommendations for as many users as possible is another desired property. 
Explainability. The reliability of recommendations is not obvious to a user unless they can be reasonably justified. This may affect the usability of the entire recommendation module on a website. It also raises wider ethical concerns around algorithmic opacity and behavior manipulation.

Serendipity. It is important to ensure that the system suggests relevant items beyond those that the user would have found anyway. Serendipitous recommendations are those that are unexpected in the context of the query, yet still interesting to the user.

A separate class of multi-criteria RS [2] has emerged to optimize and evaluate multiple properties of a recommender system at the same time.

\section{Evaluation metrics}

The accuracy of a recommender system is usually assessed not in absolute rewards, but in relative measures that take values from 0 to 1 (the higher the better). To evaluate an $N$-sized recommendation list $L(q)$ generated for a query $q$, we calculate rewards or true positives (TP) by comparing recommendations $L(q)$ to the test set $T(q)$ containing the known "ground truth" items for the active user (or session) that issued the query. This comparison also gives us the related counts of false positives (FP), true negatives (TN), and false negatives (FN), summarized in the contingency table of Figure 3.

By constructing contingency tables for the whole set $Q$ of queries, we can compute useful retrieval metrics, such as precision and recall at the chosen cutoff $N$ :

$$
\begin{gathered}
\text { Precision } @ N=\frac{1}{|Q|} \sum_{q \in Q} \frac{T P_{q}}{T P_{q}+F P_{q}} \\
\text { Recall } @ N=\frac{1}{|Q|} \sum_{q \in Q} \frac{T P_{q}}{T P_{q}+F N_{q}}
\end{gathered}
$$




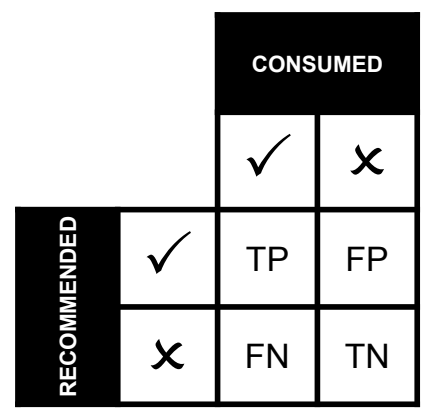

Figure 3: Contingency table

These metrics show how many rewards we get in relation to the size of the recommendation list (precision), or the size of the test set (recall). Because precision and recall trade-off against each other, they are often reported via their balanced harmonic mean, known as the F1-measure:

$$
F 1 @ N=\frac{1}{|Q|} \sum_{q \in Q} \frac{2 \cdot \text { Precision }_{q} @ N \cdot \text { Recall }_{q} @ N}{\text { Precision }_{q} @ N+\text { Recall }_{q} @ N}
$$

Since the above metrics are calculated at a fixed cutoff, they suffer from instability and may not average well [125]. The fact that the size of the test set varies from user to user impacts the ability of precision and recall to attain their maximum values. A metric called R-precision circumvents this problem by calculating precision at the corresponding recall level for each query:

$$
R \text {-precision }=\frac{1}{|Q|} \sum_{q \in Q} \text { Precision }_{q} @|T(q)|
$$

This metric therefore allows to estimate the actual accuracy of a recommendation algorithm [173].

It is a known fact that top- $N$ rankings introduce a position bias, according to which the probability of a click on an item 
decays with its rank in the list [43, 75]. The above evaluation metrics cannot accommodate this bias. For this reason, top- $N$ recommender systems are often evaluated using special top-weighted ranking measures. One of them is mean average precision (MAP), which takes the arithmetic mean of precision at all available recall levels:

$$
M A P @ N=\frac{1}{|Q|} \sum_{q=1}^{|Q|} \frac{1}{|T(q)|} \sum_{i=1}^{N} \text { Precision }_{q} @ i
$$

where: Precision $_{q} @ i=0$ if $L_{i}(q) \notin T(q)$.

Another popular top-weighted measure is normalized discounted cumulative gain (NDCG). If $r_{i} \in\{0,1\}$ is the reward obtained from displaying an item at rank $i$ of $L(q)$, then NDCG can be computed as follows:

$$
N D C G @ N=\frac{1}{|Q|} \sum_{q=1}^{|Q|} \frac{D C G_{q}}{I D C G_{q}}
$$

where: $D C G_{q}=\sum_{i=1}^{N} \frac{r_{i}}{\log _{2}(i+1)}, \quad I D C G_{q}=\sum_{i=1}^{\min (N,|T(q)|)} \frac{1}{\log _{2}(i+1)}$

Other measures often used in the RS evaluation include AUC [27], MRR [42], ERR [38], ARHR [50] etc.

\section{Content-based filtering}

As the name implies, content-based filtering [155] relies on item content features to produce recommendations, which could be textual descriptions, tags, and various attributes (metadata). The content features are used to create a joint representation of items and users in the system. A user vector encoding his/her preferences inferred from the content tokens of previously consumed items is matched to candidate items via some similarity function. 
The representation is traditionally based on the vector space model with TF-IDF token weighting [122]. The TF-IDF measure is the product of term frequency (TF) and inverse document frequency (IDF), which for each token $t$ in item $i$ are defined as follows:

$$
T F_{t, i}=\frac{f_{t, i}}{\max _{t^{\prime}} f_{t^{\prime}, i}}, \quad I D F_{t, i}=\log \frac{|I|}{1+|\{i \in I: t \in i\}|}
$$

where: $f_{t, i}$ is the frequency of occurrences of token $t$ in item $i$.

The normalized TF-IDF weight is given by:

$$
w_{t, i}=\frac{T F_{t, i} \cdot I D F_{t, i}}{\sqrt{\sum_{t^{\prime}} T F_{t^{\prime}, i}^{2} \cdot I D F_{t^{\prime}, i}^{2}}}
$$

The item-to-item or user-to-item affinity can then be computed using, for example, cosine similarity between weighted token vectors:

$$
\operatorname{sim}_{u, i}=\frac{\sum_{t} w_{t, u} \cdot w_{t, i}}{\sqrt{\sum_{t} w_{t, u}^{2}} \cdot \sqrt{\sum_{t} w_{t, i}^{2}}}
$$

Content-based recommender systems have a number of advantages:

- Most importantly, they are able to operate under cold-start and suggest items with no prior interaction history.

- They produce interpretable results, hence increasing system's transparency and user's trust.

- They can be augmented using the Linked Open Data (LOD) cloud (see the overview of LOD-based recommender systems in [51]).

On the downside,

- They suffer from overspecialization, i.e. producing too obvious results. 
- They tend to be less accurate than collaborative filtering (see next sub-section).

- In their basic form, they fail to capture the semantics of items. Recent approaches like Word2Vec learn semantic representations of items [138].

\section{Collaborative filtering}

Collaborative filtering $(\mathrm{CF})$ is the opposite of content-based filtering, as recommendations are generated solely from user interactions with no reliance on item metadata. Collaborative recommender systems make suggestions of items on the basis of similarities in consumption behavior of users. This approach is considered more powerful than content-based filtering and remains predominant in RS research [90]. It can also provide serendipitous discovery of items, which is difficult with pure content-based methods [153]. However, it is generally less transparent and secure due to "black box" computations and attacks using preference data. It also suffers from the coldstart problem.

The list of $\mathrm{CF}$ techniques is extensive. Popular families of algorithms include K-nearest neighbors [50, 178], matrix factorization [165, 90], association rules mining [135, 177], clustering [198, 171], factorization machines [164, 23], (deep) neural networks [87, 161], and others. The first three of them are particularly relevant to the thesis and will be briefly introduced next.

\section{K-Nearest Neighbors}

K-nearest neighbors (KNN) was the de facto standard in collaborative filtering long before the appearance of matrix factorization and deep learning on the RS horizon [9]. This technique is popular because of its intuitiveness, ease of implementation, and good accuracy. There is evidence that it is widely used on many e-commerce platforms [152], including Amazon [121]. 

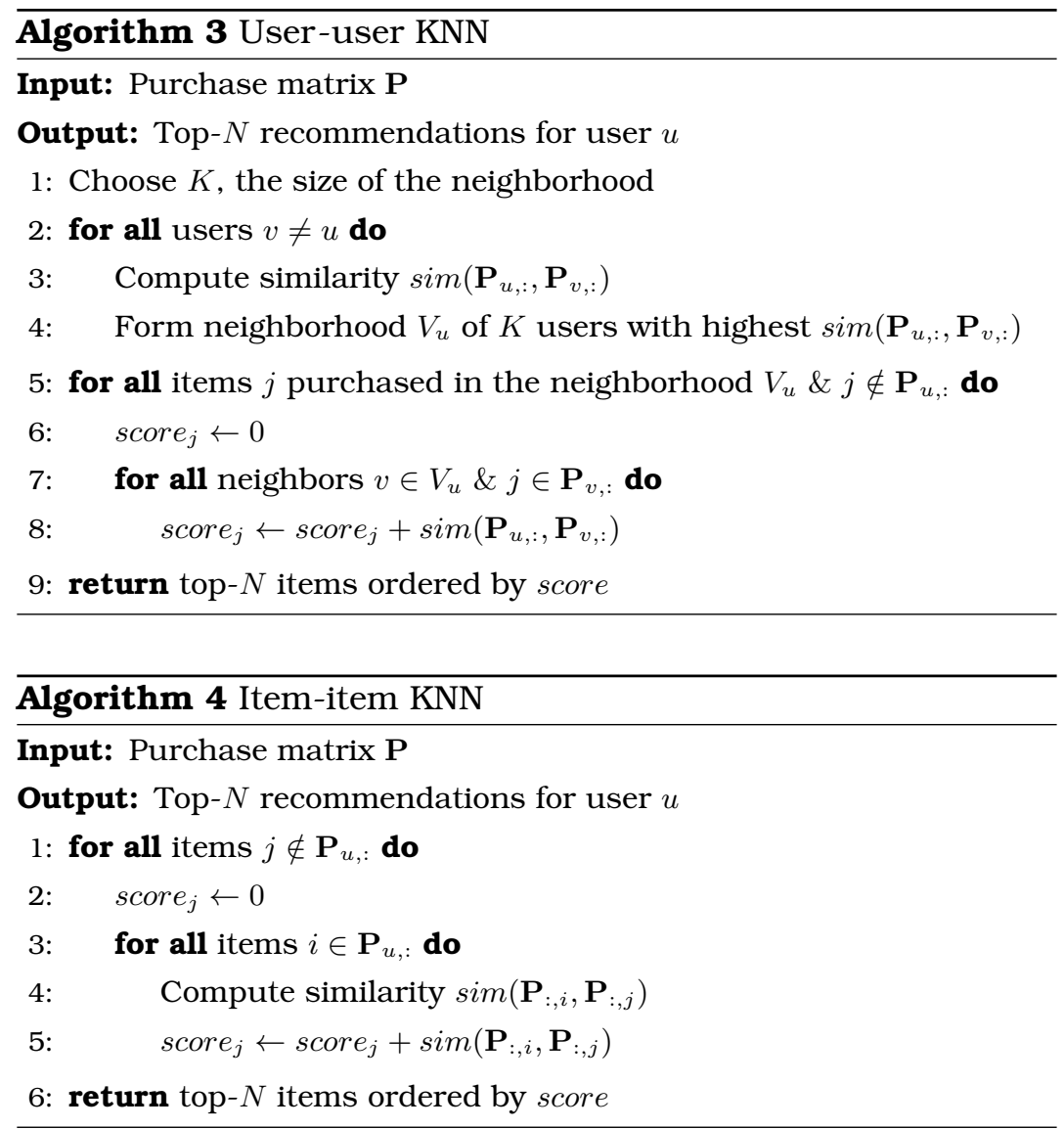

KNN does not involve any model building and hence belongs to the family of "memory-based" algorithms. The recommendations produced by KNN are based on the user-user or item-item similarity that is computed, for example, using the Jaccard coefficient of the corresponding vectors. In both cases, the recommendation space is reduced to the closest "neighbors". In user-user KNN, the size of the neighborhood is chosen by the experimenter, whereas in item-item KNN it is determined by the number of items in the user profile. The basic logic behind these two variants is presented in Algorithms 3 and 4.

The predictive superiority of one KNN method over the other seems to differ from case to case, as it follows from results 
reported in [5, 152, 203] (in favor of user-user KNN) and [178, 50] (in favor of item-item KNN). Considering the nature of the algorithm, the choice of the method is typically determined by the dimensions of the matrix: user-user KNN is preferred when there are more items that users, and item-item KNN is preferred otherwise.

Recommendations provided by KNN have good explainability (e.g. "those who bought $X$ also bought $Y$ "). They naturally handle new observations, with immediate reaction to changes in user profiles. The offline pre-computation of similarity matrices allows for very fast real-time performance. This technique can be effectively combined with other methods, such as matrix factorization [110] or deep neural nets [95]. The main drawback of KNN methods lies in their computational limits, namely scale and sparsity [33]. Since the pre-computation of similarity matrices involves multiplications of a given vector with all other vectors, the scalability becomes an issue for reasonably large datasets. Sparse data with few observations per user makes it difficult to find sufficiently large neighborhoods.

\section{Association Rules Mining}

Another technique that works intuitively well on e-commerce data is association rules mining (ARM). In fact, this approach has its roots in market basket analysis, which very closely approaches the e-commerce scenario. Association rules capture co-occurrence patterns among items in transactional data.

Consider a set of sales transactions $T=\left\{t_{1}, t_{2}, \ldots, t_{n}\right\}$, where each transaction represents a shopping basket or a user purchase history (row of matrix P). Association rules mining is done in two steps:

1. Frequent itemset generation.

Mining itemsets can be done using classical algorithms such as Apriori [4], Eclat [213], and FP-growth [78]. The frequency of an itemset $I$ is determined from its support, 
calculated as support $(I)=|\{t: I \subseteq t, t \in T\}|$.

2. Rule generation.

Each rule is a binary partitioning of a frequent itemset, taking the form $X \Rightarrow Y$. The acceptance of a rule is determined by some threshold on its confidence, calculated as $\operatorname{conf}(X \Rightarrow Y)=\operatorname{support}(X \cup Y) / \operatorname{support}(X)$.

Using this method, we can build a simple recommender system presented in Algorithm 5, where candidate items are ranked by the maximum confidence of the corresponding rules that contain them in their right-hand part (as suggested by Sarwar et al. [177]).

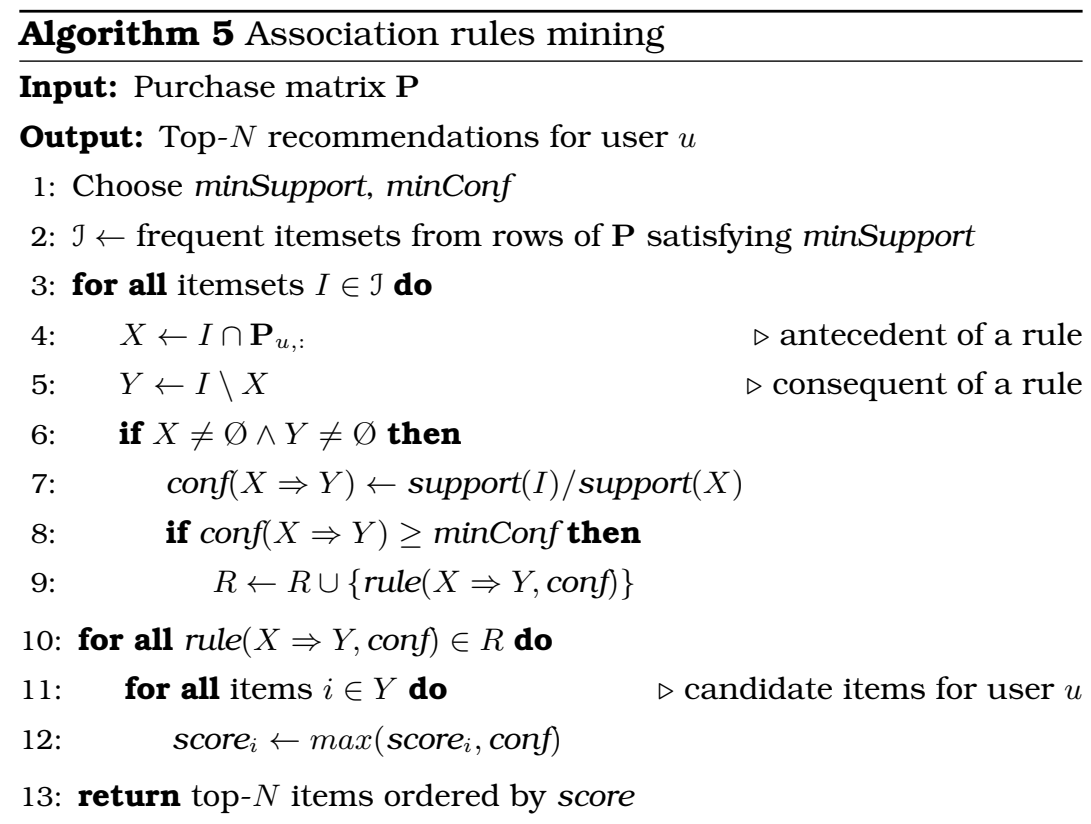

The choice of thresholds for support and confidence of rules has direct effect on the coverage and the accuracy of recommendations. The ARM technique has been successfully applied to streaming session data with notable improvements over KNN methods in terms of scalability, accuracy, and coverage [135]. 
Unlike previous methods that are based on pre-computation of item-item similarity matrices or association rules, matrix factorization (MF) belongs to the family of model-based techniques. In particular, latent factor models constitute state-of-the-art in batch-operated recommender systems and have shown excellent performance in both rating prediction [166, 174, 191, 112] and top- $N$ recommendation [97, 90, 165, 45] tasks. All these techniques are informally known as Singular Value Decomposition (SVD) models in the RS literature [45].

The key idea of these models is to project users and items of the sparse purchase matrix $\mathbf{P}^{[M \times N]}$ to the joint latent space, where users tastes and item characteristics are represented as low-dimensional dense factor matrices $\mathbf{X}^{[M \times K]}$ and $\mathbf{Y}^{[N \times K]}$. Each entry in matrix $\mathbf{P}$ can then be approximated via the inner product of the corresponding vectors in the latent space, i.e. $\hat{p}_{u i}=\left\langle\mathbf{x}_{u}, \mathbf{y}_{i}\right\rangle=\mathbf{x}_{u}^{T} \mathbf{y}_{i}$. The objective of matrix factorization is thus to find the approximation that minimizes the loss function $L(\mathbf{X}, \mathbf{Y} \mid \mathbf{P})=\sum_{u, i}\left(p_{u i}-\left\langle\mathbf{x}_{u}, \mathbf{y}_{i}\right\rangle\right)^{2}$. If $p_{u i}$ is unobserved, a common strategy is to set $p_{u i}=0$. In addition, each event can be weighted according to the number of observations, as suggested by $\mathrm{Hu}$ et al. [90]. A plausible weighting scheme is $w_{u i}=1+\alpha\left(\# p_{u i}\right)$, where $\alpha$ is a tunable parameter controlling the confidence level increase. This results in the weight matrix $\mathbf{W}^{[M \times N]}$. It is also common to add a form of $l_{2}$ regularization with a tunable parameter $\lambda$ to prevent overfitting. The loss function becomes:

$$
\begin{aligned}
L(\mathbf{X}, \mathbf{Y} \mid \mathbf{P}, \mathbf{W})= & \sum_{u, i} w_{u i}\left(p_{u i}-\left\langle\mathbf{x}_{u}, \mathbf{y}_{i}\right\rangle\right)^{2}+ \\
& \lambda\left(\sum_{u}\left\|\mathbf{x}_{u}\right\|_{F}^{2}+\sum_{i}\left\|\mathbf{y}_{i}\right\|_{F}^{2}\right)
\end{aligned}
$$

Equation 10 can be minimized via alternating least squares (ALS). This procedure fixes one of the latent vectors and solves for the other vector analytically using ridge regression. The final 
closed form solutions for both latent vectors are as follows [90]:

$$
\begin{gathered}
\mathbf{x}_{u}=\left(\mathbf{Y}^{T} \mathbf{W}_{u} \mathbf{Y}+\lambda \mathbf{I}\right)^{-1} \mathbf{Y}^{T} \mathbf{W}_{u} \mathbf{P}_{u,:} \\
\mathbf{y}_{i}=\left(\mathbf{X}^{T} \mathbf{W}_{i} \mathbf{X}+\lambda \mathbf{I}\right)^{-1} \mathbf{X}^{T} \mathbf{W}_{i} \mathbf{P}_{:, i}
\end{gathered}
$$

where: $\mathbf{W}_{u}^{[N \times N]}$ and $\mathbf{W}_{i}^{[M \times M]}$ are matrices with respective elements of $\mathbf{W}_{u,:}$ and $\mathbf{W}_{:, i}$ at the diagonal.

An MF-based recommender system based on the above procedure is outlined in Algorithm 6.

Algorithm 6 Weighted regularized MF recommendations

Input: Purchase matrix $\mathbf{P}$

Output: Top- $N$ recommendations for user $u$

1: Choose parameters $\alpha$ : confidence, $\lambda$ : regularization, $\epsilon$ : epochs

2: Initialize missing values in $\mathbf{P}$ to 0

3: Initialize factor matrices $\mathbf{X}$ and $\mathbf{Y}$ with small random numbers

4: Construct weight matrix $\mathbf{W}$ of observations using parameter $\alpha$

5: for all epochs $\epsilon$ do

6: $\quad$ for all users $u$ do

7: $\quad \mathbf{W}_{u} \leftarrow \operatorname{diag}\left(\mathbf{W}_{u,:}\right)$

8: $\quad \mathbf{x}_{u} \leftarrow\left(\mathbf{Y}^{T} \mathbf{W}_{u} \mathbf{Y}+\lambda \mathbf{I}\right)^{-1} \mathbf{Y}^{T} \mathbf{W}_{u} \mathbf{P}_{u,:}$

9: $\quad$ for all items $i$ do

10: $\quad \mathbf{W}_{i} \leftarrow \operatorname{diag}\left(\mathbf{W}_{:, i}\right)$

11: $\quad \mathbf{y}_{i}=\left(\mathbf{X}^{T} \mathbf{W}_{i} \mathbf{X}+\lambda \mathbf{I}\right)^{-1} \mathbf{X}^{T} \mathbf{W}_{i} \mathbf{P}_{:, i}$

12: for all items $i \notin \mathbf{P}_{u,:}$ do

13: $\quad$ score $_{i} \leftarrow \mathbf{x}_{u}^{T} \mathbf{y}_{i}$

14: return top- $N$ items ordered by score

A few important extensions to MF-based methods have been proposed for e-commerce data. One of them incorporates the item's marginal net utility to an SVD-based recommender system, according to which the attractiveness of an item to a user diminishes as its consumption increases [205]. The works of Zhao et al. [215, 216] utilize time intervals between purchases to improve the performance of matrix factorization in sequential recommendation settings. Finally, SVD can be adapted for incremental updates via a technique called folding-in [179], 
which allows to inject new vectors to the latent space without re-computing the entire model. This trick helps to improve the scalability of a system, but impacts the accuracy of recommendations.

\section{Multi-arm bandits}

The multi-arm bandit (MAB) family of algorithms is a newer and less researched direction in recommender systems as compared to content-based or collaborative filtering. Unlike these classical approaches, multi-arm bandits directly attack the sequential nature of RS, as they are designed for reinforcement learning tasks.

The stochastic MAB setting is defined as follows. The agent is placed in an environment comprised of slot machines with a total of $K$ actions (also called "arms"). Each arm has its own fixed probability of success, which is unknown to the agent. At each time step $t$, the agent is allowed to choose one action (pull an arm), whose outcome determines the reward $r_{t} \in\{0,1\}$ that the agent receives. The goal of the agent is to choose a policy $\pi$ that will maximize the total reward after $T$ steps, i.e. $r_{T}=\sum_{t=1}^{T} r_{t}$. To do so, the agent has to balance between exploiting the empirically optimal action and exploring unknown actions. This explore-exploit dilemma lies at the core of the multi-arm bandit theory. Algorithm 7 illustrates the operation of a stochastic MAB with Bernoulli-distributed rewards.

The function $f_{t}^{(\pi)}\left(r_{a}, n_{a}\right)$ determining the choice of action is defined by policy $\pi$. For example, it could be one of the following:

- $\varepsilon$-greedy

Based on a pre-defined parameter $\varepsilon$ and a random sample $p \sim \mathcal{U}(0,1)$, the function becomes:

$$
f_{t}^{(\pi)}\left(r_{a}, n_{a}\right)= \begin{cases}r_{a}, & \text { if } p<\varepsilon \\ p, & \text { otherwise }\end{cases}
$$




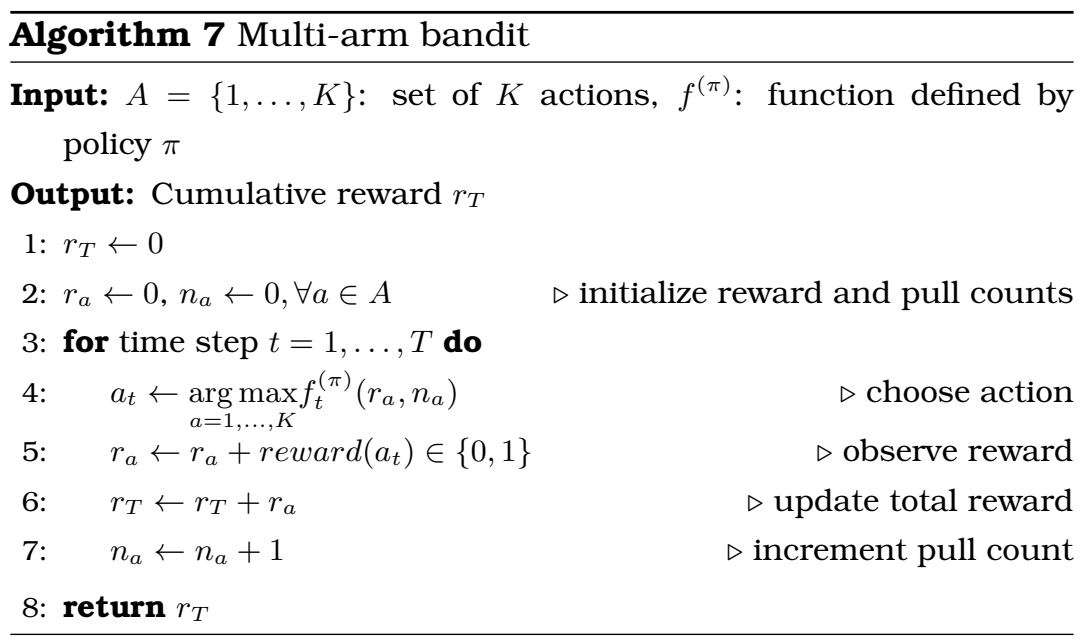

\section{- $U C B$}

For each of the first $K$ steps, UCB explores new action. After that, it continuously chooses an action deterministically, which maximizes:

$f_{t}^{(\pi)}\left(r_{a}, n_{a}\right)=r_{a}+\sqrt{\frac{2 \ln t}{n_{a}}}$

\section{- Thompson Sampling}

The choice of an action depends on its probability of being optimal, which is sampled from the Beta distribution:

$f_{t}^{(\pi)}\left(r_{a}, n_{a}\right) \sim \operatorname{Beta}\left(r_{a}+1, n_{a}-r_{a}+1\right)$

It is not hard to see that the above algorithm directly fits the sequential evaluation protocol of Algorithm 2. In Algorithm 7, one could treat each time step as a user query and each action as a candidate item for recommendation. The reward is then returned in response to the user feedback on recommendation (e.g. clicked/not clicked, purchased/not purchased). The resulting cumulative reward is considered "the unique good metric to evaluate the recommendation algorithm" [74]. It is common to extend this setting to a contextual bandit problem [117, $37,192]$, where the choice of an action also depends on the observed context vector representing the features of item/user 
at the current time step. This is intuitively sensible, since the perceptions of different users on the same item can vary significantly [117]. Huang \& Lin [91] study contextual bandits with delayed reward attribution, which is a typical scenario in many practical applications.

The classical MAB of Algorithm 7 is suitable for suggesting single items (e.g. an advertisement or a news article), but it does not natively handle top- $N$ rankings that are so common in e-commerce. One possible solution is to assign a separate bandit to each recommendation slot of a top- $N$ list, as it is done in [114]. This approach is known as ranked bandits. The obvious drawback of using independent bandits is that they are myopic with respect to user feedback and hence take longer to converge than a single bandit solution [123]. A way to address the top- $N$ ranking problem with a single MAB is to allow pulling

multiple arms at each round, for example ranked by $f_{t}^{(\pi)}\left(r_{a}, n_{a}\right)$. This type of MAB is called multiple-play bandit and has been studied in [109, 123]. Another emerging approach is based on ensemble learning, where the bandit algorithm decides which recommendation model to choose for filling the slots in the top$N$ ranking via exploring the potential of untested models and exploiting the predictive power of the already tested ones [192, $62,31]$.

\section{Hybrid filtering}

It is worth noting that best results can often be achieved when different algorithms are either combined in a single model or complement each other to reach a consensus. The success of these approaches has been witnessed in recommendation competitions such as the Netflix Prize contest or KDD cups [192].

The first approach is usually referred to as hybrid RS. Many such recommender systems take advantage of heterogeneous data sources, such as content features, collaborative patterns, and user demographics, to derive richer representations for 
modeling user behavior. This is a classical way of tackling coldstart $[182,153,46,6]$. One of the well-established techniques that enables easy blending of various data sources is factorization machine [164]. It was used in [70] to combine customer and product characteristics with behavioral data to build a hybrid recommender system for e-commerce. Blending is even possible with purely collaborative filters, for example by combining matrix factorization with neighborhood models [110, 58] or bandit algorithms [101, 218]. Fusion of user feedback types - explicit and implicit - has also proved effective [111, 116].

The second approach is known as ensemble RS. The idea behind this method is to train a number of component recommenders, whose individual outputs are aggregated for the final prediction. The aggregation is typically done by taking the average or the (weighted) majority vote [192]. This allows to reduce the variance of learning algorithms on unseen data. Ensembles are often much more accurate than the individual predictors that they are comprised of [54]. One of the most vivid examples of this is the recommendation ensemble of BellKor's Pragramatic Chaos that won the grand prize of the Netflix contest [8]. Other successful examples in the RS practice include ensembles of matrix factorizations [48, 211], multi-arm bandits [192], and mixed predictors (KNN, SVD, Restricted Boltzmann machine, etc.) [94, 15].

\section{Ethics in recommender systems}

Law, ethics, and morality

As the societal impact and the degree of algorithmic sophistication of recommender systems continue to increase, it is worthy to study these systems from an ethical perspective. Obviously, RS practitioners have an obligation to adhere to policies prescribed by legislation. However, laws alone are not sufficient to protect users from the wide range of undesirable side effects of using recommender systems and other decision-making ma- 
chinery. Unlike legislation, which has clear political bounds, ethics aim to protect core (or intrinsic) values shared by all humans regardless of their cultural, political, or geographical borders. Among these values are autonomy, freedom, security, knowledge, and resources [136]. Understanding the difference between law and ethics is, in essence, knowing to distinguish between what one has a right to do (legally, that is), and what is right to do [41].

The discipline of ethics is concerned with studying morality, which can be defined as "a system of rules for guiding human conduct, and principles for evaluating those rules" [194]. The purpose of this system is "to prevent harms and evils" [69] and "to promote human flourishing" [194]. Narrowing this down to the field of RS, we can define recommendation ethics as "the study of the moral system of norms for serving recommendations of products and services to end users in the cyberspace." [151]. Cyberspace here encompasses all possible domains of recommender systems.

\section{Recommenders as moral agents}

Why studying recommendation ethics is interesting or necessary? The reason lies in the fact that recommender systems represent agents that act autonomously on behalf of the respective bodies who deploy them, thus inheriting their moral agency. Therefore, at least theoretically, RS can be thought of as a type of artificial moral agents [204]. Such agents share certain moral competencies and abilities with human decision makers [88]. Whereas they may not be entirely equivalent to human moral agents, we claim that they can have "functional morality" by satisfying two criteria: (a) autonomy, and (b) sensitivity to ethical values [204]. Furthermore, autonomous recommendation agents can be regarded as moral agents because they are (a) sources of moral action, and (b) can cause moral harm or moral good [64]. In terms of Moor's framework [137], 
the current state of (the very few) ethics-aware recommender systems ascribes them to either "ethical impact agents", or "implicit ethical agents". The former type is the weakest sense of a moral agent, which merely has ethical consequences to its actions. We provide numerous examples of such consequences in paper III. The latter type of agent employs basic ethical intelligence, which is prescribed by some in-built moral rules and principles. There is an evidence that such rule-based implicit ethical agents have begun to appear on the RS horizon (e.g. [186, 193]).

Moral responsibility

Being morally responsible for taking an action, such as giving recommendations, is conditioned on two criteria: causality and intent [194].

The principle of causality implies that some agent $A$ is held responsible for action $X$ if $A$ caused $X$, regardless of the intent. For example, consider a recommendation agent being trained on historically biased data, resulting in discriminatory results (e.g. a dating agent that learns to prevent African Americans from getting recommendations of partners that belong to a different race [201]). Even though the developers of such an agent did not have any intent to discriminate, they are nevertheless morally responsible for the outcome.

On the other hand, an agent can be also held responsible for the mere fact of immoral intent, even when the actual harm has been avoided. We can make a case when the RS operators do not act in the best interest of their users, for example by prioritizing recommendations of excess inventory rather than personalized product selections that the user expects from the system. Even if such a recommendation goes unnoticed, the designers of the system are morally culpable because of their intentions to explicitly bias the system in their own interest. 
Principles of accountable algorithms

Moral responsibility is part of a broader "robust and intuitive notion of accountability" [143], which means that an agent, or a group of agents are answerable for a caused harm [144]. According to FATML, the five principles of accountable algorithms are as follows [53]:

Responsibility. Reveal possible ways of redress for adverse individual or societal impacts of an algorithm-driven decision mechanism, and designate an internal body responsible for the timely settlement of such issues.

Explainability. Provide comprehensible, even to non-technical users and stakeholders, explanations of data and algorithms employed in automated decision making.

Accuracy. Identify, record, and make available all sources of error and uncertainty in data and algorithms, which can be used to understand their implications and frame mitigation strategies.

Auditability. Disclose necessary information in the form of documentation, API, or terms of use, which would enable interested third parties to perform monitoring, checking, and criticism of the algorithm's operation.

Fairness. Take necessary steps to prevent discriminatory or unfair algorithmic decisions with respect to different demographic groups (race, gender, etc.).

These principles make it easier for RS developers to reason about the moral accountability of their artefacts as they proceed through various development stages. Paper III is dedicated to discussing specific issues that arise on each of these stages. 


\section{METHODOLOGY}

No amount of experimentation can ever prove me right; a single experiment can prove me wrong.

- Albert Einstein

This section details our methodological approach that we follow in each research paper included in the thesis. We also outline the role of Apptus Technologies in this research.

\section{Collaboration with Apptus Technologies}

Through our collaboration with Apptus Technologies ${ }^{6}$ - a Nordic leader in e-commerce merchandising - we were able to get access to real-world shopping session data to conduct our research. At the time when this research was initiated (early 2014), public datasets with e-commerce session data were virtually non-existent, and their availability today is still limited (4 such datasets have been recently reported in [160]). The contribution of Apptus Technologies to the research presented in this thesis is threefold:

- Two datasets containing shopping sessions from books and fashion domains were provided for experimentation. Both datasets are used in paper I and paper II.

- The Apptus' eSales development platform was provided for developing prototypes and running simulations.

- The joint research on a session-based recommender system was conducted, whose results are reported in paper II.

To comply with the Apptus' code of ethics, the analysis of proprietary datasets was performed on company premises. This was done to eliminate the risk of any accidental leakage of data belonging to Apptus' clients. Furthermore, all session data

\footnotetext{
${ }^{6}$ http: //www . apptus.com
} 
were anonymized to protect the personal privacy of data subjects.

\section{Choice of methods}

To answer the research questions of this thesis, we apply four research methods: (1) experiment, (2) design science, (3) survey, and (4) literature review. The connection of these methods to the research papers and the research questions is illustrated in Table 1.

Table 1: Research methods

\begin{tabular}{lll}
\hline Method & Paper & RQ \\
\hline Experiment & $\mathrm{I}, \mathrm{II}$ & $1,1.1,2.1,2.2$ \\
Design science & $\mathrm{I},(\mathrm{II})^{7}$ & 2 \\
Literature review & $\mathrm{III}(\mathrm{II})^{7},(\mathrm{II})^{7}$ & 3 \\
Survey & $\mathrm{I}, \mathrm{II}$ & $3.1,1,1.1$ \\
\hline
\end{tabular}

The appropriateness of each method was assessed on the basis of two criteria defined by Oates [145]:

- the method enables the researcher to find an answer to the research question.

- the method enables the researcher to present the evidence and the conclusion, and to argue convincingly that a new knowledge has been created.

As it can be seen in Table 1, each research paper applies more than one research method. This was necessary to meet the multiple objectives of each paper. The third column of Table 1 shows that in one of the cases (RQ 1 and its sub-question RQ1.1), two different methods were used to answer the same

${ }^{7}$ secondary method 
research question. This is a mixed research method called triangulation, which is seen as "a vehicle for cross validation when two or more distinct methods are found to be congruent and yield comparable data" [96]. It is used to capture a more holistic and complete portrayal of the subject under study.

Next, we present each of the methods separately and detail how they have been applied in our research.

\section{Experiment}

Experiment is a research method that explores cause and effect relationships, aiming to prove or falsify a hypothesis about the link between a factor and an observed outcome [145]. To formulate a hypothesis, a causal factor (independent variable) and an observed/measured quantity (dependent variable) need to be identified. In our research, the experimentation strategy translates to determining how, and to what extent, the use of a certain recommendation algorithm (or some modification thereof) affects the pre-defined evaluation measure(s).

The specific type of experimentation we employ in this thesis is a simulation experiment. The design of such an experiment involves the construction of a simulation model (or simply a simulator) in such a way that it closely mimics a real-world system [93]. The two simulation models used in our experiments are based on Algorithms 1 and 2, namely batch and sequential evaluation protocols. In these studies, an experimental unit represents the result obtained from a single run or a batch, which is later averaged or aggregated to yield an output value [175]. Since experiments cannot prove anything with absolute certainty, they are typically repeated multiple times, under varying conditions [145]. In our sequential simulator that is used in paper II, the aggregated result is obtained after averaging the outputs of 10 simulation runs with different random seeds. In our batch simulator (paper I), this repetition is achieved by means of cross-validation (as was explained in 
section Evaluation methods).

In paper I, experiments are performed to obtain answers to research questions 1.1 and 1.2. The experiments designed for RQ1.1 aim to compare several recommendation algorithms in terms of their accuracy and running time. The outcomes of these experiments are triangulated with the results of the survey to obtain a clearer perspective of the studied problem from different angles. In the second series of experiments, the timespan of training data is used as an independent variable to establish its effect on the evaluation metrics (RQ 1.2).

Paper II largely relies on experimentation, which is used for both comparative evaluations of different algorithms (much like paper I), and for demonstrating the impact of various modifications of an algorithm on the measurements of interest.

\section{Design science}

Design science aims to create technology-oriented artefacts that serve human purposes [126]. More specifically, it seeks to "create innovations that define the ideas, practices, technical capabilities, and products through which the analysis, design, implementation, and use of information systems can be effectively and efficiently accomplished" [85]. Design science artefacts can be of four types: constructs (vocabulary and symbols), models (abstractons and representations), methods (algorithms and practices), and instantiations (implementations and prototypes) [86]. The artefacts produced in our research belong to the last two types, since they are both formalized as algorithms, and implemented as prototypes. In paper II, we devise a bandit-based recommendation algorithm and prototype it on the eSales platform provided by Apptus Technologies. In paper I, design science is merely used as a secondary research method, since it does not directly address any research question. We devise and implement a search method for optimizing hyperparameters of recommenders that we experimentally eval- 
uate in the paper.

Design science is essentially a problem-solving paradigm [145, 85]. The design science research cycle iterates between five steps [199], which we outline and illustrate below on the example of paper II:

1. Awareness. This step gives birth to a research problem, identified in relevant practice or research. The output of this phase is some form of proposal for a new research effort. In our research, we proposed to study the design of a bandit-based recommender system, which could overcome certain challenges encountered in the real world, namely non-stationarity and varied availability of actions (component predictors).

2. Suggestion. This is a creative step, where a novel solution or a tentative idea is envisioned for a problem at hand. Based on the identified challenges, we put forth our idea of extending Thompson Sampling to build an ensemble recommender system that could accommodate the above challenges.

3. Development. In this phase, the idea is implemented according to the type of the artefact. Here, our proposal was materialized into a functional prototype recommender system running on the eSales platform.

4. Evaluation. This step assesses the artefact on some implicit or explicit criteria outlined in the first step. It usually reveals deviations from expected results, in which case the newly gained insights are communicated to another round of Suggestion (step 2). This iterative workflow was used to incrementally improve the prototype via repeated simulations. Prototypes are typically used for "proof-of-concept" evaluations, whose purpose is to demonstrate that the proposed solution has certain properties, or that it behaves in a particular way under certain condidtions [145]. 
5. Conclusion. The research cycle ends when the results are perceived as satisfactory and "firm", meaning that the observed behavior can be easily re-invoked if needed, and any anomalous behavior serves as ground for further research. Our study concluded with convincing evidence that the proposed ensemble method outperformed strong baselines on three different datasets.

\section{Survey}

The survey methodology has three main characteristics [156]:

- It is used to obtain specific quantitative descriptions of the population in a standardized way.

- It collects information from people by asking them structured and pre-defined questions.

- It focuses on a fraction of the population - a sample, whose size is large enough to enable statistical analyses.

This methodology was applied in paper I and paper III. Our design choices in each case were guided by the survey's objective. In paper I, the goal of the survey was to reveal important characteristics of commercial implementations of recommender systems. Thus, the data collected through the survey represented facts about the studied phenomenon. In paper III, survey was used to study the feasibility of the proposed "ethical toolbox" for RS users. Here, the collected data captured user opinions on specific ethical issues. In both cases, web-based questionnaires were employed as a data generation method. This method allows to reach many people across the globe quickly and at a low cost [145]. The studies were crosssectional, i.e. focusing on a particular "snapshot" of a population in time [40].

Other important design considerations have to do with the choice of sample frame and size, as well as sampling technique. In paper I, our sample frame consisted of numerous 
e-commerce platforms that featured recommendation engines. Because of the anticipated difficulties in obtaining businesssensitive insights, we aimed for meeting the minimally acceptable threshold of 30 companies as a final sample size. This is considered de facto standard for studies with low estimated response rates [145]. Indeed, the measured response rate was $12.4 \%$, with 31 total responses. The limited sample size was compensated by triangulating the results of the survey with our experimental results. We used random sampling as our sampling technique. The online questionnaire was communicated to respective development teams via email or private chat session. In paper III, the target group was not companies, but individuals. Since acquiring people's opinions was clearly an easier task than surveying companies, we opted for a larger sample, which yielded 224 responses. At the same time, we restricted our sample frame to students and faculty members, and only those who confirmed their experience of using recommender systems. The questionnaire was disseminated through Facebook groups of numerous European universities, whose members were free to participate in the survey. This sampling technique is known as self-selection sampling [145]. The weakness of this method lies in the reduced representativeness of a sample. To compensate for this weakness, we used probabilistic random sampling of target groups. Whereas the resulting sample is still skewed towards academic environments, we deem it acceptable for a feasibility study. In a deployed prototype application, however, a more representative sample would be desired.

\section{Literature review}

Literature review is the necessary means to provide the foundation and the conceptual framework for a research [145]. It was used as a secondary research method in each paper of the thesis to identify gaps in RS literature and motivate the formu- 
lated research goals. In paper III, it was also used as a primary research method to address the ethical challenges in recommender systems.

In this thesis, we adopt a rigorous, evidence-focused literature review methodology proposed by Hagen-Zanker \& Malett [76]. This method follows the key principles of a systematic literature review (rigor, transparency, and replicability), at the same time enabling more flexible retrieval and analysis mechanism. This mechanism entails three separate activities:

1. Database search using pre-defined search strings. As suggested by Wohlin [208], we initiated our search using Google Scholar ${ }^{8}$ to alleviate the bias towards any specific publisher.

2. Snowballing, which was applied to the initial literature selection identified in the previous step to drive the study further. We performed snowballing by systematically examining both the references list of the paper, and the citations to the paper. These techniques are known as backward and forward snowballing, respectively [208]. Snowballing is very useful for identifying influential, seminal work within the field of study [76].

3. Grey literature capture. Relevant material can often be found in sources beyond traditional peer-reviewed publications, such as academic journals and conferences. The inclusion of the so-called "grey literature" sources (e.g. working papers, reputable blogs, technical reports, etc.) makes it possible to find relevant material that has just been released and cannot be picked up via snowballing. It contributes to the breadth, relevance, topicality, and utility of a literature review [76].

In paper III, the above methodology was applied to each of the RS development stages. At each stage, the literature review

\footnotetext{
${ }^{8}$ https://scholar.google.se/
} 
revealed existing ethical issues and known countermeasures. Being evidence-focused, our literature study largely relied on the identification of existing examples of moral dilemmas in the RS practice to support the narrative. The findings of the literature review were summarized in the form of an ethical recommendation framework presented in paper III.

\section{Limitations}

The application of the chosen research methods in practice entails certain risks and limitations that are worth acknowledging.

An obvious limitation of our experiments lies in the fact that they are performed on recorded data. This means that no matter how well an experiment is designed, the algorithm being tested cannot influence the user's behavior. This impacts the evaluation of reinforcement learning algorithms (like the one presented in paper II), since they learn to adapt to a static snapshot of an environment that has been generated by some other means. To reliably measure the actual reinforcement effect of a recommender system, it has to be tested online (e.g. through A/B testing).

The evaluation of stochastic bandit policies presented in paper II necessitates averaging the measurements obtained via multiple runs of the same algorithm, since the results of each run are not deterministic. The number of runs $(n=10$ in our case) affects the statistical reliability of the estimates.

In paper I, the chronological mode of evaluation is based on a particular dataset split. Although the strategy itself is more realistic than random sampling, it may be less reliable as compared to cross-validation that averages over several splits.

When it comes to surveys, there are two main limitations we have been confronted with. The first one has to do with the sample size. As mentioned earlier, our final sample in paper I consists of 30 companies, which is an acceptable mini- 
mum for surveys with very low response rate. This low rate is attributed to the fact that companies were asked to reveal some details of their algorithms. This is clearly a challenging request, since many companies take serious precautious to protect their trade secrets. We compensate this by using the triangulation methodology, as explained earlier.

The second survey of our thesis (paper III) has much higher sample size (224), since it is addressed at individuals. An even higher number of participants would make this user study stronger.

The second limitation has to do with the representativeness of a sample. In paper I, the sample is heavily biased towards commercial platforms, whose number is almost twice that of open-source platforms. However, this distribution is simply a characteristic of the obtained responses, and not of our sampling strategy.

In paper III, on the other hand, we deliberately choose a sample that focuses on academic environments, and hence does not represent the entire population. This is a typical example of convenience sampling that is often practised in small-scale research [49] to obtain a sample in a fast and cheap way. In our case, this sampling method can be justified on the ground that our survey is merely a feasibility study and not a rigorous user study. Furthermore, we find it likely that such a sample increases the probability that a user is familiar with recommender systems (as compared to an average population).

As far as the literature review is concerned, we are aware of the pitfalls associated with using the aforementioned "grey literature" sources. To mitigate the risk of running into false facts and misconceptions, we evaluate the credibility of each source by verifying the reputation of the respective author/resource before adding it to our reference list. 


\section{PAPER OVERVIEW}

In this section, we give a brief overview of included papers in terms of aims, findings, and contribution. 
Paper I: Comparative Evaluation of Top- $N$ Recommenders in Ecommmerce: an Industrial Perspective

Problem. This paper focuses on the gap between recommender systems proposed by the scientific community, and those deployed by industrial e-commerce platforms. We also investigate the evaluation and training of recommendation algorithms when timestamps of events are available.

Findings. The main finding of this research is that industrial recommender systems in e-commerce are rather simple in nature, with techniques such as best-seller recommendations, $\mathrm{K}$-nearest neighbors, and association rules mining being predominant. We also empirically show that the recency and the sequence of events is important in training and evaluating recommender systems.

Contribution. We contribute with the important insight into real-world recommendation engines of numerous e-commerce platforms, concluding that the adoption of sophisticated recommendation algorithms in industry is rather slow. A side artefact of our research is a hyperparameter tuning method based on golden section search. 
Paper II: Ensemble Recommendations via Thompson Sampling: an Experimental Study within E-commerce

Problem. In this paper, we focus on the problem of item-toitem recommendations, for which a number of behavioral and attribute-based predictors are provided to an ensemble learner. We also address the problem of varied action availability and non-stationary rewards in Thompson Sampling. Finally, we investigate the effects of priming the sampler with prior knowledge, such as past event data or product catalog.

Findings. We find Thompson Sampling to be a top-performing algorithm in comparison to six other popular bandit policies. We also find a way to adjust Thompson Sampling for nonstationary environments with "sleeping" actions in the context of top- $N$ recommendations. In addition, our experiments reveal that priming Beta priors in Thompson Sampling using side information is possible, but not crucial for the algorithm performance.

Contribution. We contribute by devising a Bandit-driven Ensemble for E-commerce Recommendations (BEER), which operates in sequential item-to-item recommendation scenarios. We also show how this algorithm can be primed with side information, attaining an additional accuracy gain. 


\section{Paper III: Towards an Ethical Recommendation Framework}

Problem. The goal of this paper is to provide a holistic view on various ethical challenges that complicate the design and use of recommender systems. We also examine the possibility of introducing a set of user-adjustable controls that would allow users to control various morally sensitive components of a recommender system.

Findings. We identify and exemplify multiple moral issues that arise on various stages of a recommendation process, from user profiling to A/B testing. Through the performed literature study, we conclude that many of these issues remain unresolved, and also overlooked in the RS literature.

Contribution. The contribution of this paper is two-fold: (a) We outline an ethical recommendation framework, which summarizes RS-related ethical challenges and maps them to RS design stages; (b) We propose and preliminarily evaluate an "ethical toolbox" for RS users as a means to make recommender systems ethics-aware by design. 


\section{CONTRIBUTIONS}

This section summarizes the contributions of the thesis. Each of the three research questions of the thesis are accompanied with sub-questions that focus on more specific aspects of the respective RQ. We revisit each of these questions and elaborate on the findings of the related papers.

\section{Research question 1}

The first research question addressed the gap between scientific contributions in the field of RS and the realities of the industrial sector of RS. It was formulated as follows:

RQ1. How to assess the receptiveness of the e-commerce domain to recent algorithmic advances in the field of recommender systems?

This question is motivated and investigated in paper I. As the first step to answering the question, it is important to understand and emphasize the specificities of the e-commerce domain in relation to the movie domain that seems to get the most attention in the RS community. A vastly influential work is the one of Pradel et al. [158], who point out that "the abundance of academic papers on collaborative filtering should be contrasted with many critical aspects of the design of commercial recommender systems which remain mostly undocumented". In particular, it is noted that e-commerce datasets are characterized by very sparse purchase events and the general lack of ratings. This has led us to formulating the following sub-question:

RQ1.1. Which recommendation algorithms are suitable for e-commerce settings, where explicit ratings are inexistent and implicit information is sparse?

Our contribution with respect to the aforementioned comment of Pradel et al. is the survey of existing commercial recom- 
mender systems on three criteria: (1) algorithmic approaches, (2) input data, and (3) properties (Figure 4).
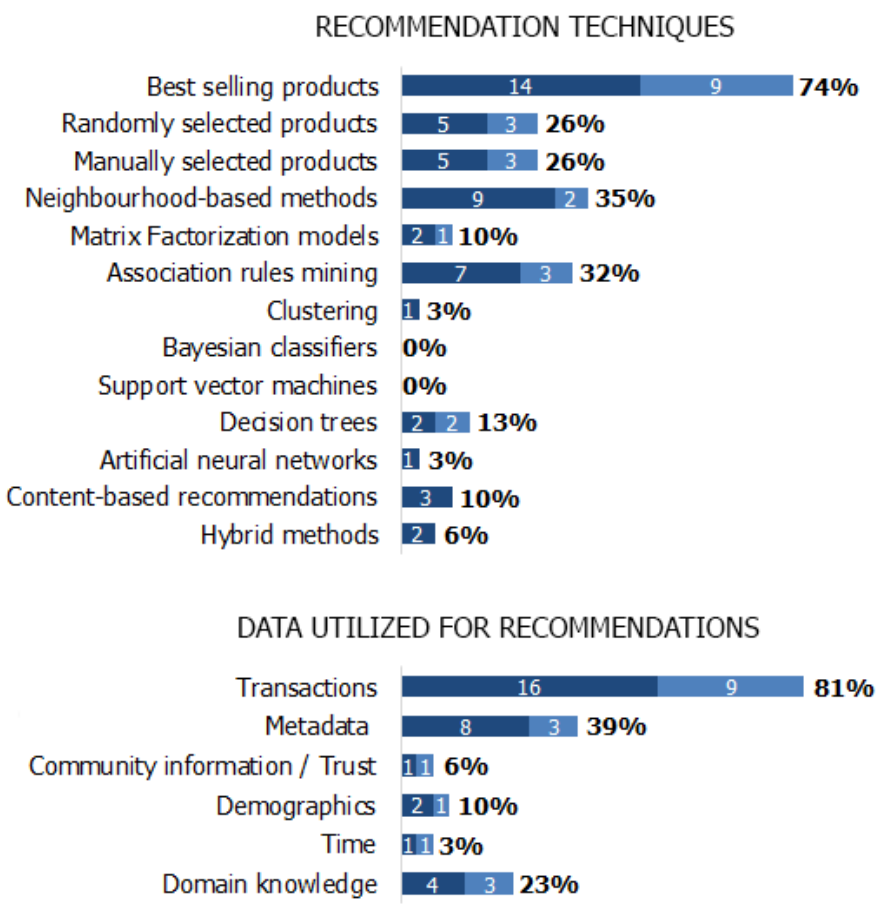

PROPERTIES OF RECOMMENDATIONS

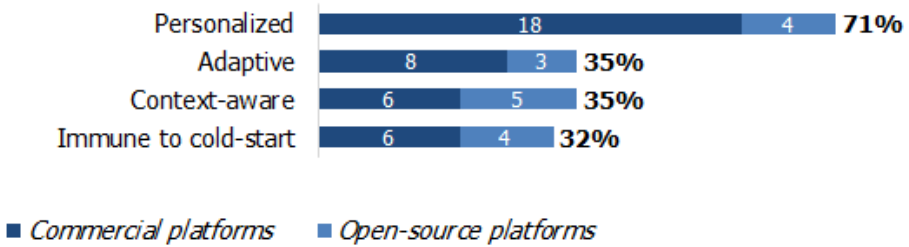

Figure 4: Characteristics of industrial recommendation engines (paper I)

Our survey reveals that the vast majority of industrial recommendation engines employ rather trivial techniques, such as recommendations of best sellers, manually selected products, and even random products. The most popular algorithmic approaches are KNN methods and association rules mining. Unsurprisingly, transactional data has been identified as the pre- 
dominant input signal, proving that collaborative filtering overshadows content-based filtering not only in the RS literature, but also in practical applications.

These findings have subsequently been compared with the outcomes of our simulation experiment, where representative techniques from three big algorithmic families - matrix factorization, neighborhood models $(\mathrm{KNN})$, and association rules mining (ARM) - are evaluated on two proprietary e-commerce datasets. The results show that the last two approaches are generally more accurate and faster on purchase data, when compared to two state-of-the-art matrix factorization methods: WRMF [90] and BPRMF [165]. Table 2 reports F1@5 measurements for different algorithms evaluated in random and chronological split modes (see paper I for more detailed results).

Table 2: $F 1 @ 5$ scores of different algorithms run on two e-commerce datasets (paper I)

\begin{tabular}{cc|cc|cc|cc}
\hline & & \multicolumn{4}{|c|}{ Collaborative filtering } & \multicolumn{2}{c}{ Baselines } \\
Split & Dataset & WRMF & BPRMF & KNN & ARM & Popular & Random \\
\hline Random & D1 & 0.1013 & 0.0676 & 0.1078 & $\mathbf{0 . 1 1 6 2}$ & 0.0458 & 0.0000 \\
Chronol. & D1 & 0.0188 & 0.0121 & $\mathbf{0 . 0 2 2 9}$ & 0.0214 & 0.0113 & 0.0014 \\
Random & D2 & 0.1322 & 0.0148 & $\mathbf{0 . 1 8 5 9}$ & 0.1813 & 0.0149 & 0.0001 \\
Chronol. & D2 & 0.0260 & 0.0130 & 0.0363 & $\mathbf{0 . 0 3 7 7}$ & 0.0114 & 0.0000 \\
\hline
\end{tabular}

These somewhat surprising findings conform to the results of the survey, and also to the ones reported by Pradel et al. [158]. As a side result, we devise a hyperparameter optimization technique based on golden section search ${ }^{9}$ as a faster alternative to the exhaustive grid search. Our experimentation on real purchase data also raises the question of how the evaluation should be carried out when timestamps of events are known. It is worth investigating this issue in a separate question:

RQ1.2. How can temporal information available in real-world datasets contribute to more realistic offline evaluation of recommender systems?

\footnotetext{
${ }^{9}$ https://en.wikipedia.org/wiki/Golden-section\_search
} 
Our first insight is the comparison between the traditional random profile splitting strategy and the time-based splitting method. We show that while the former method generally preserves the relative ranking of algorithms resulting from more realistic temporal splitting, it may overestimate the absolute accuracy of recommendations.

We also propose the expanding time window strategy, which allows to evaluate the recommender system on increasing volumes of training data, starting from the most recent events. Using this strategy, we have been able to identify the optimal training timespan for each dataset, and empirically demonstrate that training on all available data is usually suboptimal. In particular, we show that the best-seller recommender works best when trained on the most recent chunk of training data. By comparing our findings with the results of the survey, it becomes surprisingly evident that temporal information is very rarely utilized by industrial recommendation engines.

The overall contribution of paper I to the main research question (RQ1) can be summarized in the following findings:

- Industrial e-commerce platforms favor rather simple algorithmic approaches, such as best-seller recommendations, KNN and association rules mining. This attitude appears reasonable in the light of our experimental results, where more complex SVD-based methods do not seem to offer tangible advantages in accuracy and speed.

- Temporal information is important for both evaluation and training of recommender systems. However, there is an evidence that the adoption of time-aware recommender systems in industry is slow.

\section{Research question 2}

The insights gained from the previous research motivate the use of simple recommendation components (e.g. best-seller 
lists, co-purchased items, etc.) in an ensemble, and evaluating it in more realistic sequential settings. The following research question is formulated:

RQ2. How to build an ensemble of elementary recommendation components with non-stationary rewards and varied availability?

The solution we propose in paper II models each recommendation component as a bandit arm, with some pre-defined policy orchestrating the arm selection process by balancing between exploration and exploitation. The two complications reflected in RQ2 stem from the fact that individual predictors (a.k.a. "weak learners") may have irregularities in their reward sequences, and may also stay silent for certain queries. In MAB terminology, this translates to a sleeping bandit problem with non-stationary rewards. Our main contribution is the development of a bandit-driven ensemble based on Thompson Sampling policy, which is capable to accommodate the above requirements. In particular, we introduce the dynamic partitioning trick, which divides the output of each component predictor into disjoint sets of items with (relatively) fixed probability of reward. In addition, the arm selection process is modified to only allow arms with non-empty responses. The results of Table 3 show how important these modifications are for the operation of the proposed recommender system. This is our main contribution to RQ2.

Lastly, we adjust Thompson Sampling for the top- $N$ ranking problem by employing a multiple-play mechanism. With the above adjustments, the articulated bandit ensemble for ecommerce recommendations (BEER for short) can be driven by any standard bandit policy designed for stationary environments.

Our choice of Thompson Sampling policy is motivated by its proven practicality in modeling complex online problems [71], and its strong theoretical guarantees [100]. Still, it is worth 
Table 3: Standard vs. modified Thompson Sampling (paper II)

\begin{tabular}{|c|c|c|c|}
\hline Agent & Recall & Precision & NDCG \\
\hline \multicolumn{4}{|c|}{ Books } \\
\hline Thompson Sampling (SB+DP) & $\mathbf{0 . 0 5 8 1} \uparrow 83.3 \%$ & $0.0210 \uparrow 94.4 \%$ & $0.0716 \uparrow 94.6 \%$ \\
\hline Thompson Sampling (SB) & $0.0562 \uparrow 77.3 \%$ & $0.0202 \uparrow 87.0 \%$ & $0.0660 \uparrow 79.3 \%$ \\
\hline Thompson Sampling & 0.0317 & 0.0108 & 0.0368 \\
\hline \multicolumn{4}{|c|}{ Fashion } \\
\hline Thompson Sampling (SB+DP) & $0.1734 \uparrow 21.3 \%$ & $\mathbf{0 . 0 1 5 2} \uparrow 13.4 \%$ & $\mathbf{0 . 0 4 8 3} \uparrow 19.9 \%$ \\
\hline Thompson Sampling (SB) & $0.1760 \uparrow 23.2 \%$ & $0.0142 \uparrow 6.0 \%$ & $0.0427 \uparrow 6.0 \%$ \\
\hline Thompson Sampling & 0.1429 & 0.0134 & 0.0403 \\
\hline \multicolumn{4}{|c|}{ Yoochoose } \\
\hline Thompson Sampling (SB+DP) & $0.2973 \uparrow 10.5 \%$ & $\mathbf{0 . 0 4 1 9} \uparrow 10.8 \%$ & $\mathbf{0 . 1 3 9 1} \uparrow 21.4 \%$ \\
\hline Thompson Sampling (SB) & $\mathbf{0 . 2 9 8 4} \uparrow 10.9 \%$ & $0.0409 \uparrow 8.2 \%$ & $0.1273 \uparrow 11.1 \%$ \\
\hline Thompson Sampling & 0.2690 & 0.0378 & 0.1146 \\
\hline
\end{tabular}

$\mathrm{SB}=$ Sleeping Bandit, DP = Dynamic Partitioning

comparing Thompson Sampling with other low-regret policies, primarily those based on a class of Upper Confidence Bounds (UCB) $[115,12]$. This is formulated as a separate sub-question:

RQ2.1. How does Thompson Sampling compare to other bandit policies for orchestrating the ensemble in sequential settings?

In our comparative study reported in paper $\|$, Thompson Sampling outperforms six other popular MAB policies (MOSS, $\varepsilon$-greedy, and 4 variants of UCB) on all three datasets. It appears that Bayesian approaches in general are very effective in bandit problems with complex actions, as UCB-Bayes [100] has come second in accuracy in our experiments. We believe that these findings can be of interest to the research community, in particular as an extra evidence in support of Bayesian-oriented approaches advocated in [71].

Because Bayesian methods rely on priors, it is worth exploring if we can improve convergence by assigning more informative priors than the standard Beta $(1,1)$ prior of Thompson Sampling. We formulate this as our second sub-question:

RQ2.2. How does priming the ensemble method with prior knowledge affect its performance and how can such priming 
be done?

We propose two sources of information for priming Thompson Sampler: (a) past user sessions, and (b) product catalog. The first option allows us not only to obtain initial posteriors for each component of the ensemble, but also to pre-train behavioral components (e.g. co-purchased items, co-clicked items, etc.) by running the sampler through recorded data. Our results show that priming the sampler with pre-trained behavioral components has a consistent improvement in accuracy. Through option (b) we demonstrate that it is possible to achieve an accuracy gain even when no historical data are available. In this case, we can take advantage of statistical properties of the product catalog to make informed guesses about the priors of attribute-based components (e.g. same author, same genre, etc.). Our overall conclusion after experimenting with both approaches is that Thompson Sampling does not seem to enjoy particular benefits from priming Beta priors, meaning that it can handle cold-start gracefully out of the box. At the same time, our results encourage pre-training behavioral components with past transactional data, when such information is provided.

\section{Research question 3}

Our third research question addresses the issues of ethics, which emerge on various stages of the recommendation process:

RQ3. What are the ethical challenges that complicate the design and use of recommender systems, and what are the possible solutions?

The extensive literature study we perform in paper III results in the list of ethical issues, which are mapped to each RS development stage. In a broad sense, ethical concerns are categorized into the ethics of data manipulation (e.g. privacy, 
anonymity, censorship issues), modeling (e.g. algorithmic biases, behavior manipulation, discrimination issues), and experimentation (e.g. fairness, awareness, informed consent issues). We also mark known solutions to each of these issues. Our findings are summarized in the user-centric ethical recommendation framework (Table 4), promoting the paradigm of "ethics awareness by design". We envisage that this framework can serve as a roadmap for RS practitioners who implement recommendation engines in real-world applications. Prior to our research, the holistic coverage of RS-related ethical issues seems to have evaded the attention of the research community.

Aside from the theoretical exploration of recommendation ethics, we study the possibility of engaging users themselves in addressing certain morally sensitive components of a recommender system. This is formulated as a secondary research question:

RQ3.1. How to aid morality in recommender systems through user engagement?

In response to this question, we propose a practical tool for RS users, which would give them the ability to manually adjust various morally-sensitive settings of a recommender system. We coin this feature an "ethical toolbox", whose each filter corresponds to the specific stage of the articulated framework. For example, the initial user profiling stage allows users to restrict the creation and the maintenance of a user profile, whereas the last testing stage allows them to withdraw themselves from A/B testing experiments, and reset the recommendation engine to its "default" algorithm. The feasibility study we conduct in the frame of this research has a positive outcome, with 4 out of 5 proposed filters being preferred over alternative methods of handling a particular moral issue. This result motivates further research towards the prototype implementation of the toolbox with subsequent user testing. 


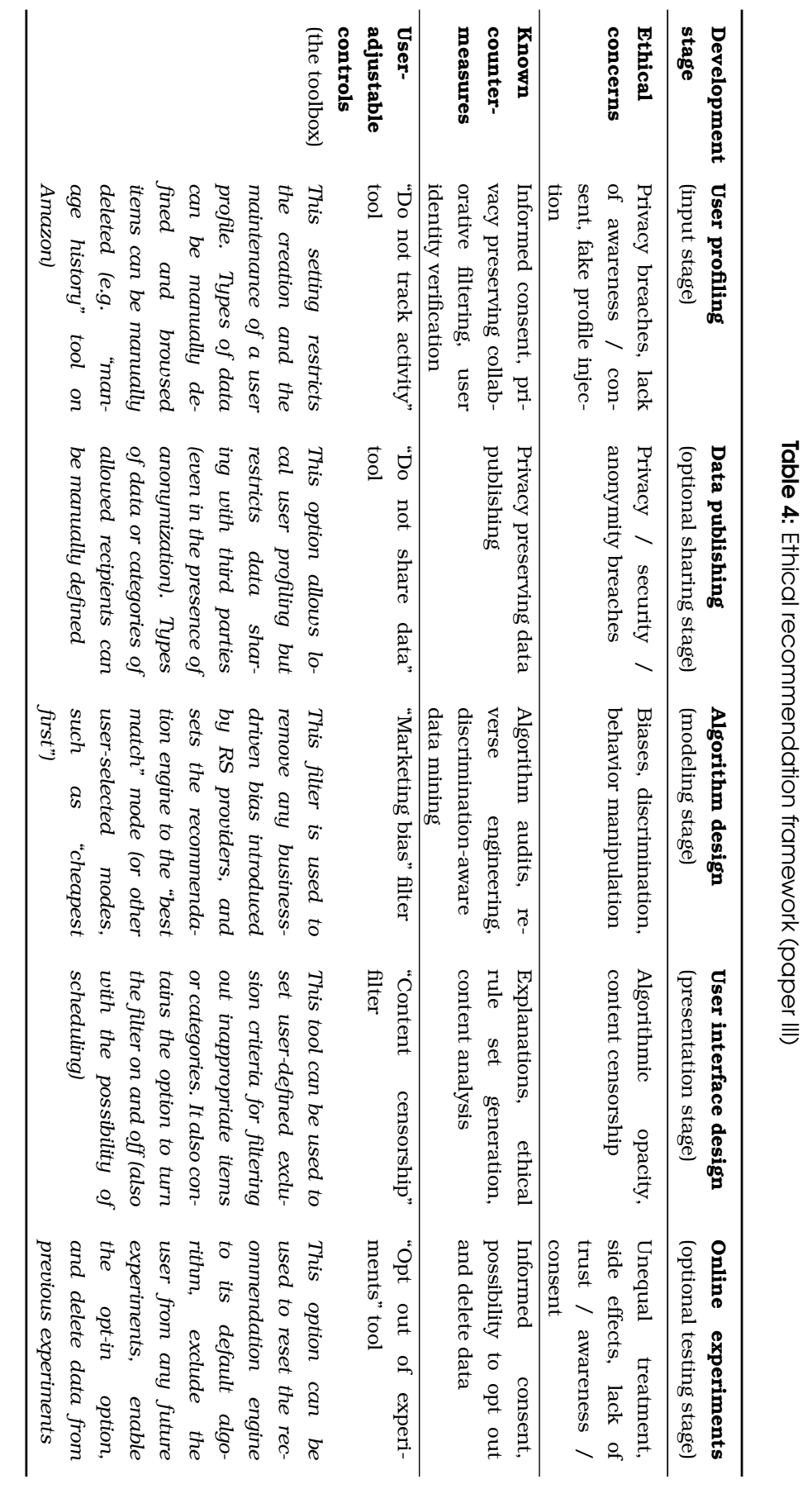




\section{CONCLUSIONS}

\section{Thesis summary}

This thesis focused on the practical side of recommender systems, i.e. how these systems are designed and evaluated for deployment on e-commerce platforms, and how they affect people's lives from the ethical standpoint.

With the abundance of collaborative filtering algorithms in the RS literature (especially in the movie domain), it is not at all clear whether the proposed methods are suitable for industrial settings. The valuable insight we obtained from examining more than 30 existing e-commerce recommendation engines led to the conclusion that the industrial sector seems to favor simple algorithmic approaches. Indeed, a short browsing session on Amazon would reveal that the classical "those-whobought-also-bought" recommender is still actively used despite the years of RS research. This also resonates well with the Netflix' business decision not to implement the prize-winning algorithm due to unjustified engineering efforts [8].

Still, the fact that the first two places of the Netflix Prize contest were won by ensembles, deserves serious consideration. These two observations (algorithmic simplicity \& the power of ensembles) motivated our effort to devise a bandit-based ensemble recommender system comprised of elementary predictors. Multi-arm bandits are attractive because they fit the sequential nature of user interactions on a website. The sequential evaluation of recommender systems has started to gain ground in recent years (e.g. [74, 160]). The ensemble model we propose in paper II is the main algorithmic contribution of this thesis. It has a number of advantages for a prospective e-retailer:

- Scalability, because bandit arms are modeled as predictors (as opposed to single items), and hence scale well with 
the growing product catalog.

- Extensibility, because it is easy to switch between bandit policies, and add new component recommenders on the fly, which get automatically explored by the bandit.

- Adaptability, because the system dynamically reacts to non-responsive predictors, and changes in reward probabilities.

- Explainability, because elementary predictors capture simple associations between items (e.g. co-purchases), and hence are easily interpretable.

It could be argued that the proposed system did not only exhibit attractive algorithmic properties, but also solved important ethical issues that had to do with transparency and user profiling. First, the ability of a system to explain each item in the top- $N$ recommendation list enabled it to overcome the issue of algorithmic opacity and help to build user's trust in the system [84]. Second, the "item-to-item" recommendation scenario could preclude privacy breaches associated with maintaining rich user profiles.

Investigating other RS-related ethical issues became another major focal point of this thesis. By following the typical recommendation pipeline, we identified and exemplified a number of ethical concerns that emerge on RS development stages. Addressing these issues often means resolving challenging tradeoffs, such as user privacy vs. personalization, data anonymization vs. data utility, informed consent vs. experimentation bias, and algorithmic transparency vs. trade secrets. As an alternative to existing normative and technological solutions, we proposed a set of tools for manual adjustment of morally sensitive components of a recommendation engine. The feasibility of such a tool was supported by the positive results of our user study. All the above were packaged and presented as an 
ethical recommendation framework for aiding the development of ethics-aware recommender systems.

\section{Future work}

The proposed "ethical toolbox" for RS users was well received by the participants of our feasibility study. However, we are aware of the possible "control paradox" - a situation where users express their willingness to have control over their data, but do not make the effort of exploiting this control in practice [105]. Therefore, the next step in this direction would be to implement and evaluate a prototype of the proposed toolbox, which would allow us to draw more reliable conclusions about its usefulness and applicability. Another issue that deserves deeper investigation is price discrimination. This topic is highly relevant for the e-commerce domain. Our initial analysis revealed the number of research efforts on price-personalized recommender systems $[217,129,98]$, in which little or no attention is given to the moral impact of personalized pricing. In our future work, we intend to study the wider problem of discrimination-personalization trade-off in recommender systems. In particular, it is worth investigating the application of existing machine learning methods adjusted for discrimination awareness with respect to protected classes (gender, race, religion, etc.). This research would bring closer the ethical and the algorithmic aspect of recommender systems. When it comes to the latter, we plan to continue our exploration of reinforcement learning ensembles. This time, we would like to take advantage of the full range of available data, i.e. transactions, metadata, descriptions, and images. A possible way of combining this information is to take a Word2Vec-inspired approach (e.g. similar to [141]). We also want to explore the possibility of adapting state-of-the-art reinforcement learning algorithms (e.g. DQN [134], A3C [133]) to e-commerce recommendation scenarios. 
PART II

INCLUDED PAPERS 


\title{
PAPER I
}

Paraschakis D., Nilsson B.J., and Holländer J.

"Comparative Evaluation of Top- $N$ Recommenders in E-commerce: an Industrial Perspective"

\begin{abstract}
We experiment on two real e-commerce datasets and survey more than 30 popular e-commerce platforms to reveal what methods work best for product recommendations in industrial settings. Despite recent academic advances in the field, we observe that simple methods such as best-seller lists dominate deployed recommendation engines in e-commerce. We find our empirical findings to be well-aligned with those of the survey, where in both cases simple personalized recommenders achieve higher ranking than more advanced techniques. We also compare the traditional random evaluation protocol to our proposed chronological sampling method, which can be used for determining the optimal time-span of the training history for optimizing the performance of algorithms. This performance is also affected by a proper hyperparameter tuning, for which we propose golden section search as a fast alternative to other optimization techniques.
\end{abstract}

Published in Proceedings of the 14th International Conference on Machine Learning and Applications (ICMLA), IEEE, 2015 


\section{Introduction}

An intelligent recommender system (RS) is a powerful selling tool of any contemporary e-commerce platform. According to Forrester ${ }^{10}$, product recommendations account for up to $30 \%$ of e-commerce sites' revenues. That was already apparent in 2006, when Amazon ${ }^{11}$ claimed that $35 \%$ of its sales were triggered by recommendations [127]. The same year, Netflix ${ }^{12}$ announced its famous million-dollar prize for improving its recommendation engine. This contest has spurred active research within the field, yielding a variety of recommendation algorithms over the past decade. Interestingly, the prize-winning algorithm was never put to real use: Netflix concluded that the measured accuracy gains "did not seem to justify the engineering effort needed to bring them into a production environment" [8]. This fact motivated us to explore how receptive e-commerce platforms are to academic advances in the field of recommender systems. The connection between research contributions and industrial solutions has rarely been in focus of RS literature. As Pradel et al. [158] noted, "case-studies are necessary to better understand the specificities of purchase datasets and the factors that impact recommender systems for retailers". Our work is such a case study.

Originating from the Netflix problem, the vast majority of proposed recommenders have been designed for rating prediction. In e-commerce, however, rating products is not commonly practised even when this functionality is supported by a shopping platform. The problem thus boils down to predicting a purchase rather than a rating score. Purchase prediction based solely on transactional history is the common approach known as collaborative filtering (CF). In the absence of explicit ratings, transactional datasets become binary and are referred to as implicit feedback datasets. When it comes to missing data, im-

\footnotetext{
${ }^{10}$ http: //www . forrester. com

${ }^{11}$ http: //www. amazon.com

${ }^{12}$ http: //www. netflix.com
} 
plicit feedback is ambiguous: if a customer has never bought a particular product, it is not always a sign of negative preference (e.g. the product might be out of stock). That is why algorithms designed for implicit feedback often model heuristics of relationships between observed and unobserved events in a dataset, as we shall see in Section MF-based methods. This direction is less researched than movie ratings, partly due to the lack of publicly available e-commerce datasets. In our experience, retailers are reluctant to release their sensitive purchase data. Another factor that differentiates these datasets from movie ratings is the extreme sparsity of data: a typical retail store only sells a fraction of its product catalogue.

Evaluation of recommender systems in industrial context deserves special attention. Whereas the main academic interest appears to be the accuracy of predictions, industrial recommenders are meant to be optimized for revenues. As Aiolli [5] pointed out, the choice of an accurate predictor is not all that is required to build a good recommender. Other evaluation criteria that play an important role in e-commerce are coverage of product catalogue, utility and serendipity of recommendations, adaptivity and scalability of an algorithm, etc. [75]. Since our work aims at a comparison between algorithms and not a rigorous testing of a particular recommender, we only consider accuracy and speed as our evaluation criteria. The most reliable way of testing a deployed RS is to perform an online evaluation (e.g. A/B testing), which may however be too costly, time-consuming and therefore often infeasible in practice. That is why customer behaviour is usually simulated offline from the recorded purchase data. The choice of a proper user modelling strategy is a crucial factor, which must provide a realistic estimate of the real performance of an algorithm. Numerous approaches for such modelling have been discussed in [75]. In this paper we propose one such technique for offline evaluation. 
The contributions of this work are the following:

1. We conduct a survey of deployed recommendation engines in the e-commerce domain. This is done to measure the extent to which recent academic achievements in the field of recommender systems are adopted by real-world applications. To the best of our knowledge, this is the first study of this kind.

2 . We find that the performance of simpler but faster algorithms such as association rules can often be superior to more elaborate techniques such as matrix factorization (MF). This confirms the results of other recent works on purchase data, such as [158].

3. Our experiments show that training on all available data is inferior to training on recent purchases for most algorithms. To optimize recommendation accuracy, we propose the expanding time window evaluation method for finding the optimal training time-span.

4. We adapt the Golden Section Search (GSS) algorithm for multi-dimensional hyperparameter optimization.

The paper is organized as follows. Section Methodology presents our methodology, where we describe our datasets, evaluation protocol and metrics. We then present the GSS algorithm and the survey. In Section Recommendation algorithms, we give a brief presentation of the recommendation techniques used in our study. In Section Results, we present and analyze our experimental results and survey responses. Finally, Section Conclusions concludes the paper.

\section{Related work}

A few case studies, closely related to ours, performed comparative evaluations of different families of recommendation algorithms on purchase datasets. A study by Huang et al. [92] points to the need for better understanding of relative strengths 
and weaknesses of different types of algorithms in e-commerce applications. Thus, they performed a comparison of six different algorithms and found that spreading activation and link analysis produced the best results. Just as in our study, they experimented on a fashion and a book store. A more recent case study by Pradel et al. [158] experimentally evaluated various $\mathrm{CF}$ algorithms on a dataset of a French home improvement and building supplies chain. The simple bigram rules recommender was found to yield the best overall accuracy. One important conclusion of this work is that the relative performances of algorithms depend on the setting to which they are applied.

In relation to this, both studies evaluate recommenders in two settings: complete and reduced transaction history. In [92], the reduced training set contains a fraction of randomly sampled events, whereas in [158] it contains the event history of the last two weeks of the training data. In our paper, we adopt a more flexible setting where we evaluate algorithms in various history lengths (see section Experimental protocol).

\section{Methodology}

In our study we conduct offline experiments on two real ecommerce datasets: a fashion store (denoted as D1) and a book store (denoted as D2). All experimentation is done on an Intel Xeon 2.53 Ghz PC with $12 \mathrm{~Gb}$ RAM. Our offline experiments aim to compare the performance of algorithms from the three families: $\mathrm{MF}$-based $\mathrm{CF}$, memory-based $\mathrm{CF}$, and data mining (detailed in section Recommendation algorithms). Two methods that recommend most popular and random products are used as baselines. The representative algorithms from each of the aforementioned families were selected from the MyMediaLite library ${ }^{13}$ [66] to aid reproducibility. Semantic- and contextaware methods have not been considered due to the nature of

\footnotetext{
${ }^{13}$ with the exception of the ARM recommender and the LLR-based similarity, which were implemented separately
} 
the input data, which consists solely of purchase events. All techniques are evaluated for their predictive power and speed. Our experiments are complemented with an online survey, which assesses recommendation engines of existing e-commerce platforms. Survey responses are analyzed in section.

\section{Datasets}

The summary of the two datasets is given in Table 5 .

Table 5: Summary for datasets D1 and D2

\begin{tabular}{lll}
\hline & D1 & D2 \\
\hline Domain & Fashion & Books \\
Timespan & 9 months 26 days & 3 months 10 days \\
Customers & 26,091 & 505,136 \\
Products & 4706 & 210,455 \\
Events & 78,449 & $1,623,576$ \\
Sparsity & $99.936 \%$ & $99.998 \%$ \\
\hline
\end{tabular}

Both datasets are of implicit feedback and contain a history of purchases over a period of time. Each dataset is represented as a binary purchase matrix $P^{[m \times n]}$, whose entry $P_{u i} \in\{0,1\}$ indicates whether customer $u \in U^{[m]}$ bought product $i \in I^{[n]}$ at least once. The two datasets differ in size, sparsity, type of merchandise, and timespan (the timespan is illustrated in Figure 5). 


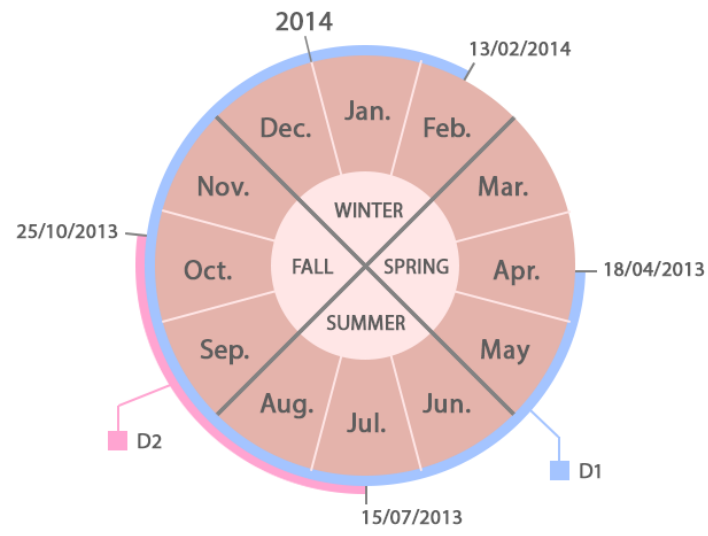

Figure 5: Timespan of datasets

The distribution of purchases per customer is given in Figure 6, which depicts the characteristic sparsity of purchase data. For our experiments, we pre-filtered the datasets to contain customers with at least 2 purchases, which is the bare minimum that enables customer profile splitting (see section Experimental protocol), while maintaining high sparsity levels.
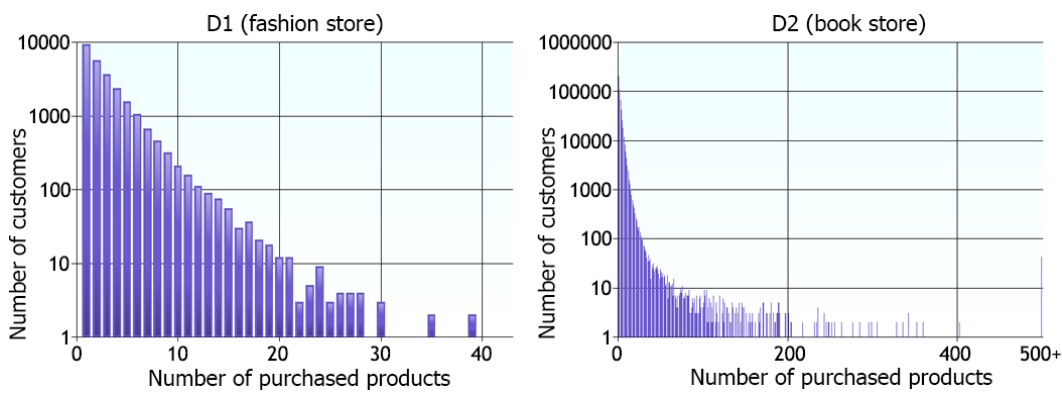

Figure 6: Distribution of purchases per customers

\section{Experimental protocol}

The common strategy for offline experiments is to split the dataset into training and test sets, where the latter simulates future purchases and contains a fraction of events withheld from the original dataset. The remaining events are kept in the train- 
ing set, which is fed to a recommendation engine that outputs a ranked list of unobserved products based on some underlying algorithm. The top- $\mathrm{N}$ products are then taken from the list as final recommendations. The choice of the split ratio between training and test sets is arbitrary and usually ranges from $80 / 20$ to $50 / 50$ percent.

In our experiments, we follow two splitting strategies:

1. Traditional approach (non-chronological split). As it is often practiced in RS literature [102, 165, 173, 177], we start by discarding "cold-start" customers with profiles below a constant threshold. In our case, we only keep those with at least 10 purchases. We then divide all customers into 5 folds for cross-validation. In each test fold, we randomly withhold half of events ${ }^{14}$ from customer profiles to build the test set. The other half of events together with the remaining folds is used for training the models.

2. Proposed approach (chronological split). The availability of timestamps allows us to attempt a more realistic setup, where we train on past events to predict future events. Furthermore, we deliberately keep all customers in place from the initial pre-filtering step, when only those with at least 2 purchases were kept. This is the shortest user profile length that can be divided in two parts (training and testing). This choice was motivated by our intention to simulate a real cold-start environment. A temporal dataset is illustrated in Figure 7.

In this setting, we set a split point on the dataset's timeline (indicated with the red vertical line), which acts as our "present". Past events located to the left of the split point are used for training, whereas future events located on the right side are used for testing. Customers whose entire profiles lie in the "future" are discarded, whereas "past customers" only appear in the training set. Keeping the split point (and hence the test set) fixed, we train the models using the expanding time

\footnotetext{
${ }^{14}$ if the number of events is odd, the training set will be larger than the test set by 1 event
} 


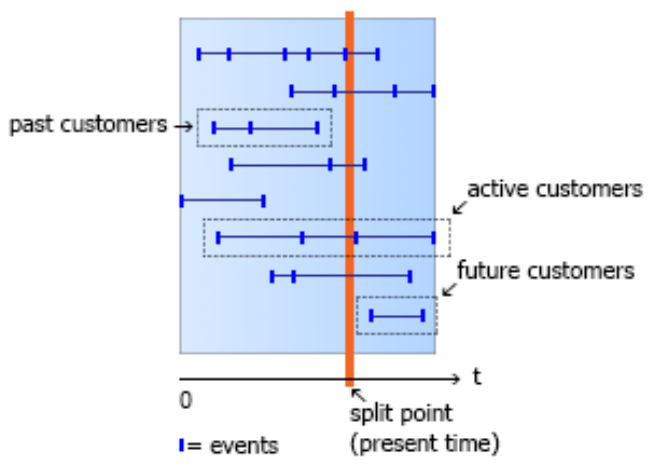

Figure 7: Representation of a temporal dataset. Horizontal lines represent customer profiles on a timeline, where purchases are marked with blue bars.

window, starting from the most recent history and then going back in time at a fixed rate up to full history. This approach allows us to determine an optimal training interval from our measurements.

In both cases, the task is to recommend products matching those of the test set. The motive for attempting both approaches was to determine whether the widely used random, non-chronological splits can reliably reproduce the same ranking of algorithms as the more realistic temporal, cold-start splits.

\section{Evaluation metrics}

Top- $N$ recommendation lists are typically evaluated in terms of their precision and recall. Precision measures the percentage of recommended products that are relevant, ${ }^{15}$ whereas recall measures the percentage of relevant products that are recommended.

Given a fixed size $N$ of recommendation list, we calculate the harmonic mean of precision and recall, known as F1-score:

$$
F 1 @ N=\frac{1}{|\mathcal{U}|} \sum_{u=1}^{\mid \mathcal{}} \frac{2 \cdot \text { precision }_{u} @ N \cdot \text { recall }_{u} @ N}{\text { precision }_{u} @ N+\text { recall }_{u} @ N}
$$

\footnotetext{
${ }^{15}$ being relevant means being a member of the test set
} 
where $\mathcal{U}$ is the set of "active customers" (see Figure 7).

In our experiments we choose $N=5$. Notably, evaluation at fixed $N$ suffers from bias introduced by unevenly distributed number of events in the test set across customers. When this number is larger than $N$, even a perfect recommender would never reach the maximum accuracy score. Therefore, metrics with fixed $N$ do not average well and can only be used to estimate the relative performance between algorithms. To tackle this problem, we also measure precision at the level of recall, known as R-precision:

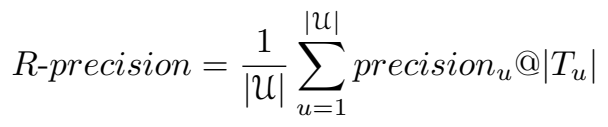

where $T_{u}$ is the test set of customer $u$.

Thus, $R$-precision adjusts for the size of the customer $u$ 's test set and hence ensures that evaluation has an attainable maximum value [173]. Thus, the aforementioned bias is eliminated and averaging across customers makes more sense [125]. As opposed to F1-score, R-precision allows to estimate the absolute quality of a recommendation algorithm [173].

The disadvantage of the above evaluation metrics is that they do not capture the utility of recommendations, making them all look equally interesting to the customer. However, usability studies show that customers are used to scan recommendation lists from top to bottom, often observing only a few products on the top of the list [75]. Therefore, we use a special ranking measure to account for a positional discount down the list, known as Mean Average Precision (MAP):

$$
M A P=\frac{1}{|\mathcal{U}|} \sum_{u=1}^{|\varkappa|} \frac{1}{\left|T_{u}\right|} \sum_{k=1}^{\left|T_{u}\right|} \text { precision }_{u} @ k
$$

where precision $_{u} @ k=0$ if product_at_position_k $\notin T_{u}$ 
Thus, MAP is the arithmetic mean of precision values across recall levels, averaged over all customers. MAP is a very popular metric in information retrieval and is favored for its discrimination and stability [125].

The above evaluation metrics are measured within the range $[0,1]$, where higher values are better.

\section{Hyperparameter optimization via Golden Section Search}

Most recommendation algorithms are affected by their hyperparameters that need to be properly "tuned" when applied to a new dataset. This is a problem of optimizing a cost function over a (multi-dimensional) configuration space. Evaluating a cost function is often computationally expensive, rendering common approaches such as manual and grid search inefficient. We address this issue by adapting a Golden Section Search (GSS) to our multi-dimensional hyperparameter optimization, under the assumption of unimodality of the objective function. In one dimension, the problem can be formulated as finding a point $x$ in the configuration space $[a, b]$ such that the objective function $f(x)$ is maximized:

$$
x^{*}=\underset{a \leqslant x \leqslant b}{\arg \max } f(x)
$$

The GSS algorithm finds $x^{*}$ through iterative shrinkage of the configuration space by sampling points between $a$ and $b$ using the inverse golden ratio $\varphi \approx 0.618$, until the resulting interval becomes smaller than the pre-set tolerance $\epsilon$ :

Given a set $\mathcal{P}$ of hyperparameters, the algorithm iterates over them and the function $f(x)$ evaluates a recommender in the current hyperparameter setting. Based on the given values of $a, b$, and $\epsilon$ of each hyperparameter, the required number of iterations can be pre-computed as follows:

$$
\# \text { iter }=\frac{\sum_{i \in \mathcal{P}} \log \frac{\epsilon_{i}}{b_{i}-a_{i}}}{\log \varphi}
$$




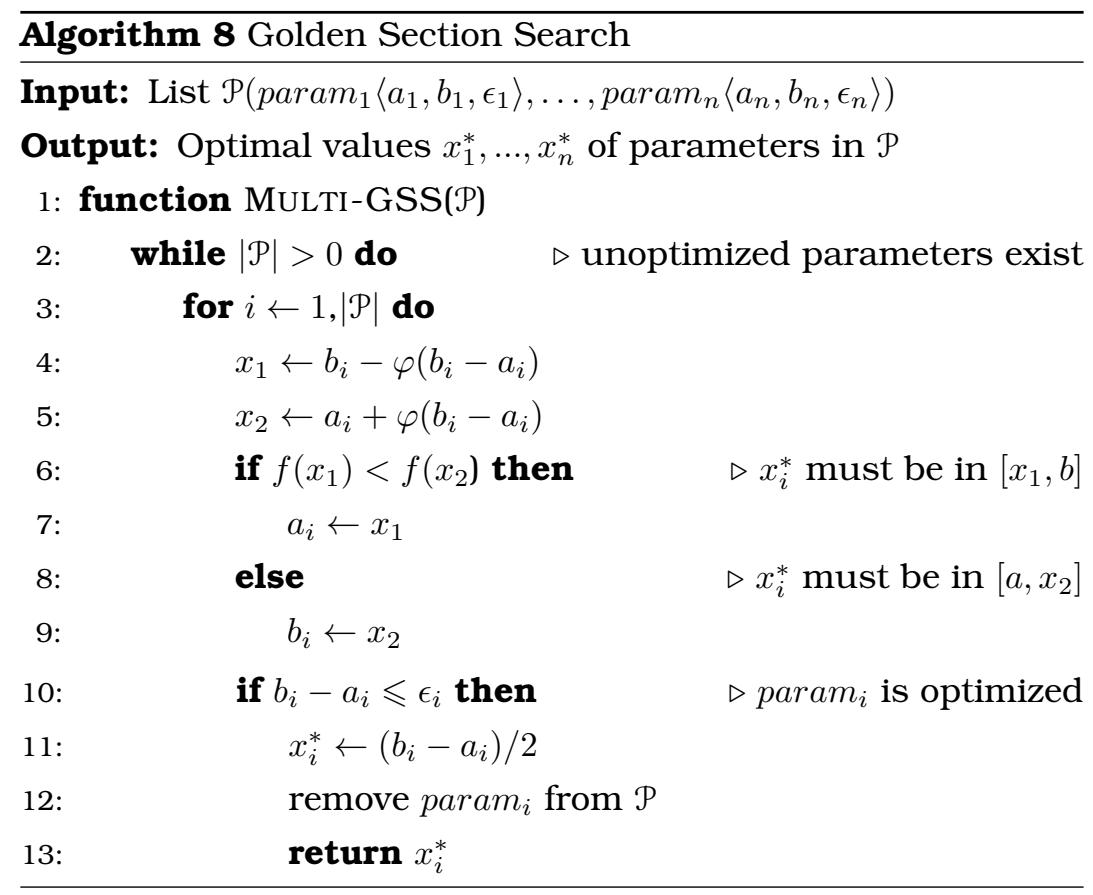

This allows for adjusting the value of $\epsilon$ prior to running GSS in accordance with the available computational resources. GSS has fast convergence because it never samples past a suboptimal point, but does not guarantee finding a global maximum when the objective function is multimodal. This leads to situations when the last iteration does not necessarily produce the highest function value. To tackle this problem, we save the values of hyperparameters every time when the evaluated function peaks at its current best value.

For tuning hyperparameters in our chronological setup, we used validation sets dating from $17 / 07 / 2013$ to $31 / 07 / 2013$ for D1 and from 29/07/2013 to 05/08/2013 for D2. We trained all algorithms on events prior to the validation events of each dataset. For the non-chronological case we validated algorithms on customers with 9 events, where random 65\%-35\% user profile splitting was done. This ensures that our validation sets are always disjoint from the test sets, where only customers with 
at least 10 events are considered.

\section{Survey of deployed recommender systems}

The survey was aimed at commercial and open-source e-commerce software vendors, who were asked to describe their recommendation engines as accurately as possible. This was done via an anonymous online questionnaire, which assessed the deployed RS on three criteria: a) properties of recommendations, b) data utilized for recommendations, and c) recommendation techniques. The selection of these techniques is based on the classification by Amatriain et al. [9], with the addition of extra (non-personalised) techniques, such as random, manual, or best selling product recommendations. Each question had a set of pre-defined answers with a possibility to select multiple options and provide a free-form answer if so desired. The questionnaire is available online at http://goo.gl/forms/ ZFEfLLIFHO.

\section{Recommendation algorithms}

In this section we give a brief theoretical foundation for various algorithms that were used in our experiment.

\section{MF-based methods}

MF-based models decompose the original purchase matrix $P$ into lower-rank factor matrices $W \in \mathbb{R}^{[m \times d]}$ and $H \in \mathbb{R}^{[d \times n]}(d \ll$ $\min (m, n))$, which capture latent relationships between customers and products. The interest of customer $u$ in product $i$ is estimated by the dot product of the corresponding factor vectors $\hat{P}_{u i}=W_{u:} H_{i:}^{T}$. The major challenge is to compute the mapping of customers and products to a joint latent factor space.

Most prevalent factorizations encountered in RS literature are based on singular value decomposition (SVD), where the mapping is performed iteratively either through the alternating least squares (ALS) or the stochastic gradient descent (SGD) 
optimization process. In our study, we examine several SVDbased algorithms which have been specifically designed for implicit feedback datasets.

WRMF The Weighted Regularized Matrix Factorization model $[90,149]$ is a state-of-the-art algorithm based on SVD, which is formulated as a least squares problem with two core components: a regularization term to prevent overfitting, and a confidence value associated with each event. The intuition behind this measure is that the confidence of observed events being positive is higher than the confidence of unobserved events being negative. One way to enforce this is to assign higher weights to positive entries in $P$, although other weighting schemes have also been discussed by Pan et al. [149]. The WRMF optimization criterion is given as:

$$
\min \sum_{u i} C_{u i}\left(\left(P_{u i}-W_{u:} H_{i:}^{T}\right)^{2}+\lambda\left(\left\|W_{u:}\right\|_{F}^{2}+\left\|H_{i:}\right\|_{F}^{2}\right)\right)
$$

where $C_{u i}$ is a confidence of event $P_{u i}, \lambda$ is a regularization parameter, and $\|\cdot\|_{F}$ is a Frobenius norm of a matrix.

The factor matrices are first initialized with normally distributed random numbers. The optimization process then alternates between updating $W$ and $H$ as follows: [149]:

$$
\begin{aligned}
W_{u} & :=P_{u:} C_{u} H\left(H^{T} C_{u:} H+\lambda I\right)^{-1} \\
H_{i:} & :=P_{: i}^{T} C_{: i} W\left(W^{T} C_{: i} W+\lambda I\right)^{-1}
\end{aligned}
$$

The advantage of ALS is that it can be easily parallelized and its running time is linear in the size of the input [90].

BPRMF The BPRMF [165] model is formulated as a ranking problem and is based on Bayesian Personalized Ranking optimization criterion (BPR-OPT). Unlike WRMF which scores single products in $P$, BPRMF optimizes for correct ranking of product pairs for each customer, i.e. the triple $\langle u, i, j\rangle$. The basic idea 
of BPR is that if customer $u$ consumed product $i$ but did not consume product $j$, then $P_{u j}<P_{u i}$ ranking-wise. The generic BPR-OPT criterion is given as [165]:

$$
B P R-O P T=\sum_{u i j} \ln \sigma\left(\hat{x}_{u i j}\right)-\lambda_{\theta}\|\theta\|^{2}
$$

where $\sigma(x)$ is a logistic sigmoid $1 /\left(1+e^{-x}\right), \hat{x}_{u i j}$ is an arbitrary function that specifies the relationships in $\langle u, i, j\rangle, \theta$ is a parameter vector of an arbitrary model, and $\lambda_{\theta}$ is a model-specific regularization parameter.

In BPRMF, the estimation of $\hat{x}_{u i j}$ is performed through matrix factorization. But since the MF model can only predict single events, the estimator is decomposed into single prediction tasks: $\hat{x}_{u i}-\hat{x}_{u j}$. The optimization is performed through SGD with bootstrap sampling of training triples using the following update rule [165]:

$$
\theta:=\theta+\alpha\left(\frac{e^{-\hat{x}_{u i j}}}{1+e^{-\hat{x}_{u i j}}} \frac{\partial}{\partial \theta} \hat{x}_{u i j}+\lambda_{\theta} \theta\right)
$$

where $\alpha$ is the learning rate.

\section{Data mining (association rules)}

Association rules mining (ARM) has been a popular instrument for market basket analysis (e.g. in supermarkets), where associations between products are established from customer purchase patterns. This technique is therefore a natural choice for recommender systems and has demonstrated good performance on real-world e-commerce datasets, as shown by Pradel et al. [158]. In its simplest form, an association rule $i \Rightarrow j$ implies that whenever a customer buys product $i$, he or she is likely to buy product $j$. The strength of a rule is measured on the basis of its confidence and support, which are calculated as follows:

$$
\operatorname{con}(i \Rightarrow j)=\frac{P_{: i}^{T} P_{: j}}{\sum_{u \in U} P_{u i}} \quad \sup (i \Rightarrow j)=\frac{P_{: i}^{T} P_{: j}}{\sum_{u \in U} \sum_{i \in I} P_{u i}}
$$


In other words, confidence is the probability of buying product $j$ given that product $i$ was bought, while support is the fraction of events where products $i$ and $j$ were bought together.

The training phase involves computation of all available rules, whose confidence values are stored in a sparse matrix of size $n \times n$. To produce recommendations for customer $u$, a set of supported rules is first identified from a customer profile. Then, unobserved products are typically ranked by either the maximum confidence [158, 177] or the sum of confidences [102] of corresponding rules. In our case, the linear combination of confidence and support was found to yield better results:

$$
\operatorname{rank}(j)=\sum_{i}^{\left|I_{u}\right|} \operatorname{con}(i \Rightarrow j) \cdot \sup (i \Rightarrow j)
$$

where $I_{u}$ is the profile of customer $u$ (e.g. the set of observed products), and $j$ is a candidate product for recommendation.

\section{Memory-based CF (K-nearest neighbors)}

Memory-based CF relies on the notion of similarity between customers or products. Thus, the key training step is the formation of a neighborhood, which becomes a source of recommendations. For a given customer, recommendations can be computed either from the neighborhood of similar customers (user-based KNN), or from the neighborhood of products similar to those he or she has bought (item-based KNN). Although the latter approach has been favored in RS literature, we found user-based KNN to be more effective on both our datasets, in line with the results of $[5,203]$.

The performance of a KNN recommender is much dependent on the similarity measure used to calculate the neighborhood. Cosine and Jaccard similarity are commonly used for this task. In our tests, the Log-Likelihood ratio proposed by Dunning [55] and adapted for CF by Owen et al. [148] produced superior results. In short, this metric estimates the probability that the overlap of events in customer profiles is not due to 
chance [148]. Let $I_{u}$ and $I_{v}$ denote two customer profiles. The Log-Likelihood ratio (LLR) is then defined as:

$$
L L R=f\left(I_{u}, I_{v}\right) \cdot\left(H\left[I_{u}, I_{v}\right]-H\left[I_{u}\right]-H\left[I_{v}\right]\right)
$$

where $f\left(I_{u}, I_{v}\right)$ is a count-dependent scaling factor, $H\left[I_{u}, I_{v}\right]$, $H\left[I_{u}\right]$ and $H\left[I_{v}\right]$ are the entropy of the joint and the marginal probabilities, respectively (refer to $[55,56]$ for details).

During training, an LLR-based neighborhood is calculated for each customer. The testing phase ranks unobserved products based on the accumulated LLR scores of each neighbor who consumed the products in question.

\section{Results}

In this section, we present the results from our experiments on datasets D1 and D2. All algorithms were tuned using GSS and then evaluated on the three metrics described in Section Evaluation metrics. After reporting the experimental results, we also present a summary of the survey responses.

\section{Experiment 1. Non-chronological split}

We first present the results from the "traditional" approach, where we have:

- test customers with at least 10 purchases

- random sampling of events (50/50 profile splitting)

- 5-fold cross-validation

The performance of algorithms in this setup is given in Tables 6 and 7. It can be seen that UserKNN and ARM outperform MF-based methods on all metrics, which is especially prominent in case of D2, where BPRMF has performance comparable to that of the most popular books recommender. This finding conforms to those reported in [5, 158].

The presented runtimes of algorithms were calculated as the sum of training and testing time. In Tables 8 and 9 the average 
running times (across training sets) are reported. The results are given in the format hh:mm:ss. Aside from the implementation details, the running time of algorithms depends on their hyperparameter values, such as $K$ for UserKNN or iterations / factors for MF-based methods. We can see that WRMF is consistently slower than other methods, despite having been trained with fewer iterations than BPRMF in all cases. We attribute it to the fact that ALS involves a least squares solution, which is more expensive than an SGD iteration. The noticeable boost in WRMF speed in Table 9 is merely because the algorithm was trained with fewer factors than in random mode (determined by the GSS).

Table 6: Scores for the random split on D1

\begin{tabular}{ccccc}
\hline Recommender & MAP & $F 1 @ 5$ & R-prec & Running Time \\
\hline WRMF & 0.0927 & 0.1013 & 0.1036 & $00: 05: 21$ \\
BPRMF & 0.0650 & 0.0676 & 0.0698 & $00: 00: 32$ \\
\hline UserKNN & 0.1000 & 0.1078 & 0.1073 & $00: 00: 05$ \\
ARM & $\mathbf{0 . 1 1 0 0}$ & $\mathbf{0 . 1 1 6 2}$ & $\mathbf{0 . 1 1 5 5}$ & $00: 00: 01$ \\
\hline Most Popular & 0.0523 & 0.0458 & 0.0468 & $00: 00: 00$ \\
Random & 0.0028 & 0.0000 & 0.0010 & $00: 00: 00$ \\
\hline
\end{tabular}

Table 7: Scores for the random split on D2

\begin{tabular}{ccccc}
\hline Recommender & MAP & F1@5 & R-prec & Running Time \\
\hline WRMF & 0.1293 & 0.1322 & 0.1363 & $07: 10: 10$ \\
BPRMF & 0.0188 & 0.0148 & 0.0187 & $00: 12: 46$ \\
\hline UserKNN & $\mathbf{0 . 1 7 9 6}$ & $\mathbf{0 . 1 8 5 9}$ & $\mathbf{0 . 1 8 5 4}$ & $00: 56: 21$ \\
ARM & 0.1774 & 0.1813 & 0.1825 & $00: 16: 24$ \\
\hline Most Popular & 0.0139 & 0.0149 & 0.0156 & $00: 07: 02$ \\
Random & 0.0002 & 0.0001 & 0.0001 & $00: 09: 09$ \\
\hline
\end{tabular}




\section{Experiment 2. Chronological split}

The second experiment was conducted maintaining the coldstart issue in the data and splitting the datasets chronologically using the expanding time window approach described in Section Experimental protocol. The set partitioning was performed on a monthly basis for D1 and on a bi-weekly basis for D2. The test set contained the last month of data for D1 and the last 2 weeks of data for D2. The training sets were of increasing length from 1 to 9 months for D 1 and from 2 to 12 weeks for D2. The different partitioning scales were chosen because of considerably shorter timespan of D2 (even though it contained much more events). Figures 8 and 9 show the performance of algorithms for varied history lengths. Purple dots indicate peaks in performance. 

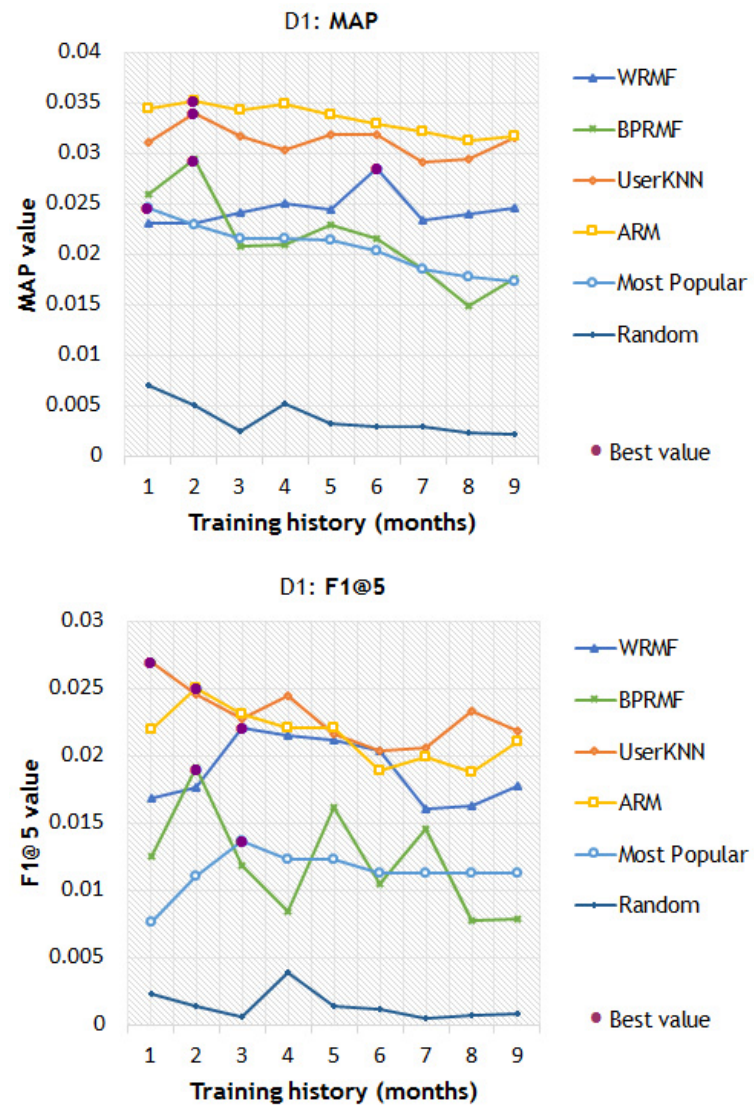

- Best value

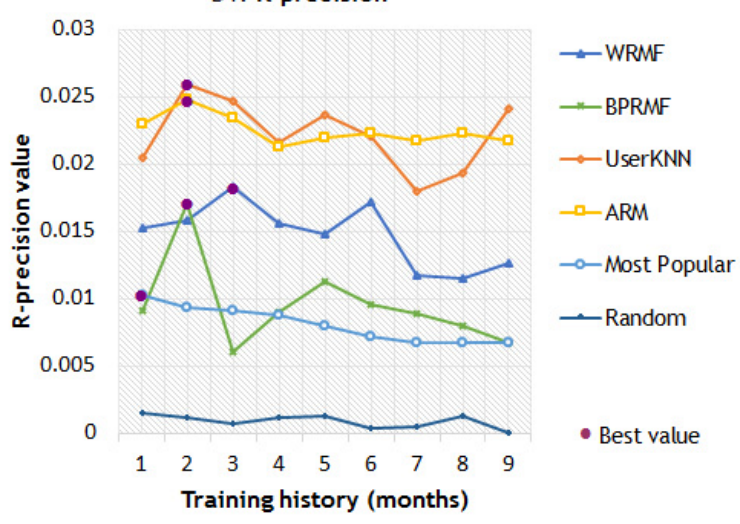

Figure 8: Chronological split of D1 with 9 training sets 
Paper I: Comparative Evaluation of Top- $N$ Recommenders in E-commerce: an Industrial Perspective
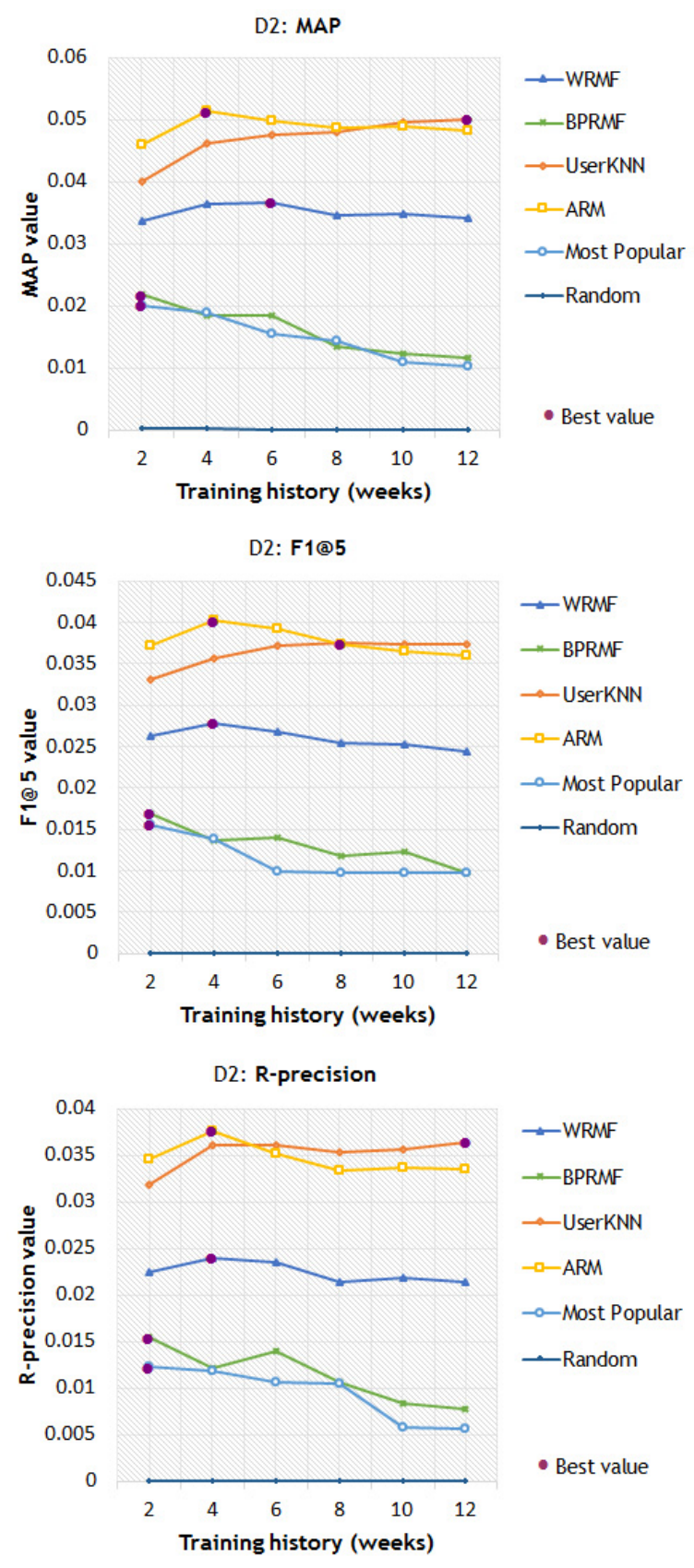

Figure 9: Chronological split of D2 with 6 training sets 
From observing the performance peaks in Figure 8, it becomes clear that the optimal training history for D1 lies within the 3 recent months, which appears logical for a fashion store. A similar trend of favoring recent events is observed in Figure 9, with the exception of UserKNN, whose performance increases proportionally with the size of the history. It can also be seen that best-seller recommendations are generally most effective when recommending the most recently sold merchandize. These observations suggest the adoption of time-aware recommenders in e-commerce, which have shown improved quality of predictions in the movie ratings domain [112].

Table 8: Average scores for the chronological split on D1

\begin{tabular}{ccccc}
\hline Recommender & MAP & $F 1 @ 5$ & R-prec & Running Time \\
\hline WRMF & 0.0244 & 0.0188 & 0.0147 & $00: 05: 27$ \\
BPRMF & 0.0214 & 0.0121 & 0.0095 & $00: 00: 06$ \\
\hline UserKNN & 0.0312 & $\mathbf{0 . 0 2 2 9}$ & 0.0222 & $00: 00: 06$ \\
ARM & $\mathbf{0 . 0 3 3 4}$ & 0.0214 & $\mathbf{0 . 0 2 2 5}$ & $00: 00: 01$ \\
\hline Most Popular & 0.0206 & 0.0113 & 0.0080 & $00: 00: 00$ \\
Random & 0.0037 & 0.0014 & 0.0009 & $00: 00: 00$ \\
\hline
\end{tabular}

Table 9: Average scores for the chronological split on D2

\begin{tabular}{ccccc}
\hline Recommender & MAP & F1@5 & R-prec & Running Time \\
\hline WRMF & 0.0351 & 0.0260 & 0.0224 & $02: 27: 10$ \\
BPRMF & 0.0160 & 0.0130 & 0.0114 & $00: 18: 37$ \\
\hline UserKNN & 0.0469 & 0.0363 & $\mathbf{0 . 0 3 5 2}$ & $00: 50: 14$ \\
ARM & $\mathbf{0 . 0 4 8 8}$ & $\mathbf{0 . 0 3 7 7}$ & 0.0346 & $00: 20: 35$ \\
\hline Most Popular & 0.0150 & 0.0114 & 0.0094 & $00: 10: 24$ \\
Random & 0.0001 & 0.0000 & 0.0000 & $00: 14: 42$ \\
\hline
\end{tabular}


Tables 8 and 9 show the performance of algorithms after averaging their accuracy scores over all training sets. The results indicate that in general, time-based splits preserve the same ranking we observed in random splits, although with less significant difference in performance scores. The low measurement values is the consequence of cold-start conditions: predicting purchases for customers with short profiles is difficult (but realistic). However, this is not the only reason. We extended our experiment 2 by keeping test customers with at least 10 purchases. We then performed a chronological split followed by a 50/50 random split (as in experiment 1). The effect we observed after evaluating algorithms in these two protocols was quite remarkable: randomizing events in customer profiles boosted the performance of some algorithms by more than 300\% (e.g. for WRMF). This observation tells that preserving the sequence of events during evaluation is crucial and that traditional random splits may produce too optimistic estimates of a recommender's accuracy.

\section{Survey responses}

31 responses were obtained in total from 20 commercial and 11 open source e-commerce platforms ${ }^{16}$. The responses to each of the 3 questions are summarized in Figure 10. Below we discuss some of the interesting findings.

Transactional data is used by more than $80 \%$ of respondents, which points to the clear dominance of $\mathrm{CF}$ over the content-based approach. At the same time, timestamps of events are neglected by most companies, which seems unreasonable in the light of our experimental findings. Other contextual sources of data have also shown low usage, even though more than a third of recommendation engines have been reported to be context-aware. This must be due to the comparatively

\footnotetext{
${ }^{16}$ Among participants who chose not to stay anonymous were VP-Cart, Ecwid, WebJaguar, YesCart, NitroSell, S3Cart, Graphflow, NationKart, Whirlwind eCommerce, and PinnacleCart
} 

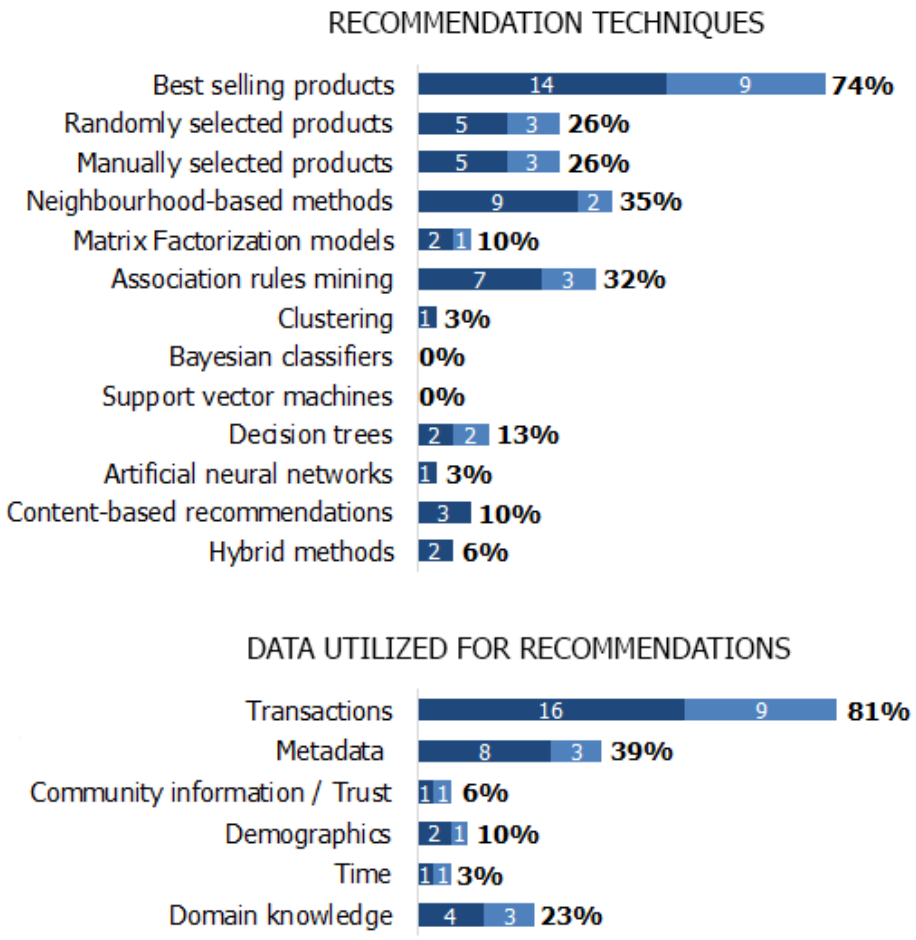

PROPERTIES OF RECOMMENDATIONS

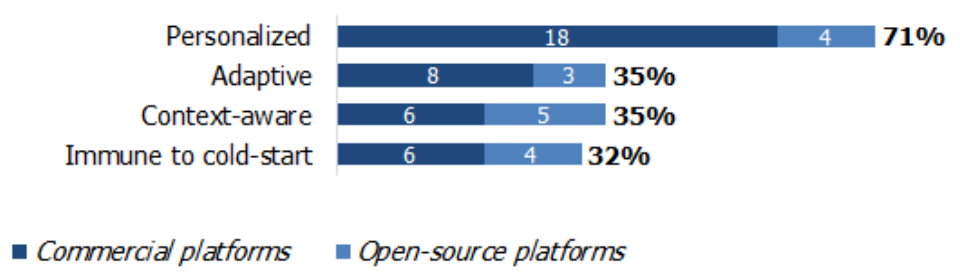

Figure 10: Survey responses

higher percentage of ad-hoc recommenders based on domain knowledge.

It seems that commercial platforms tend to put much more effort in personalization than their open-source competitors. We observe that neighborhood-based filtering and association rules mining are the most popular personalized recommenders, which totally conforms to our experimental results.

Recommending best selling products is the preferred ap- 
proach for most platforms. Indeed, our experiments show that outperforming best-seller lists can be challenging even to personalized recommenders. Furthermore, trivial methods of recommending random or manually selected products are more widespread than state-of-the-art methods, such as MF.

It appears that some platforms can be opposed to RS as such. One of the respondents argued that "if a shopper goes to all the trouble to choose the right product, it is almost insulting to link them to a page that says 'if you don't like this, then here are some recommendations.' Adding recommendations to a page that is the end result of a buying decision is a distraction and is likely to lower the rate of conversion".

\section{Conclusions}

Recommender systems is an important area of academic research and a vital revenue-generating tool in e-commerce. In this paper, we raised the question of the adoption of techniques in industry and the effectiveness of recent machine learning models for typical e-commerce settings, where explicit ratings are inexistent and implicit information is sparse.

We have shown that deployed e-commerce platforms take rather simplistic approach to product recommendations, with best-seller lists being at the heart of their engines. When it comes to personalized recommendations, the incline toward "good old methods" is not as surprising as it seems: our experiments confirm the superior performance of KNN collaborative filtering and association rules mining to more advanced MFbased methods when applied on binary purchase data. Similar findings have been reported in [158].

Whereas in academic context researchers tend to prioritize accuracy over speed (according to [19], only 11\% of RS research papers report runtimes), in industrial settings where real-time interaction with the system is employed, the time factor is undoubtedly vital. This might be another reason in favor of sim- 
pler techniques. We show that time is also important in terms of the recency and the sequence of events. Depending on the type of merchandize, e-commerce datasets exhibit varied effects of seasonality. Thus, our experiments indicate that training on all available history is in most cases not optimal. We present a time-based splitting strategy that helps to establish the optimal timespan of a training set. This optimization can lead to improved recommendation accuracy and runtime efficiency. We also show that traditional random sampling, which often implies training on future events to predict past events, is prone to producing too optimistic performance estimates due to capturing erroneous shopping patterns.

Finally, we propose fast Golden Section Search for automatic tuning of algorithms with multiple hyperparameters.

\section{Acknowledgment}

We express our gratitude to Apptus Technologies for the provided datasets and computational resources. 


\title{
PAPER II
}

Brodén B., Hammar M., Nilsson B.J., and Paraschakis D.

"Ensemble Recommendations via Thompson Sampling: an Experimental Study within E-commerce"

\begin{abstract}
This work presents an extension of Thompson Sampling bandit policy for orchestrating the collection of base recommendation algorithms for e-commerce. We focus on the problem of item-to-item recommendations, for which multiple behavioral and attribute-based predictors are provided to an ensemble learner. We show how to adapt Thompson Sampling to realistic situations when neither action availability nor reward stationarity is guaranteed. Furthermore, we investigate the effects of priming the sampler with pre-set parameters of reward probability distributions by utilizing the product catalog and/or event history, when such information is available. We report our experimental results based on the analysis of three real-world e-commerce datasets.
\end{abstract}

Published in Proceedings of the 23rd Conference on Intelligent User Interfaces (IUI), ACM, 2018 


\section{Introduction}

Dynamically responding to user queries with top- $N$ item ranking is a very common problem on the web, which pertains to search, advertising, and recommendations. A specific use case of interest is generating item-to-item (i2i) recommendations for every visited product page with the aim to maximize product sales. Such non-personalized recommendations are useful when user profiles are limited or non-existent. This is very typical of online shopping sessions, where most visitors are unidentified [196], yet keen to see meaningful recommendations already on their first page visit (e.g. on a landing page). Such dynamic environments with streaming event data and severe coldstart make conventional recommender systems impractical and call for reinforcement learning approaches, whose explorationexploitation paradigm appears very appropriate [119].

Item rankings can be generated by various predictors that utilize item consumption patterns and/or content features. Learning which predictor (or a combination thereof) is optimal in a given context in an online manner naturally translates to a multi-arm bandit (MAB) problem. In its classical formulation, a policy sequentially chooses from a finite set of actions with stationary (but unknown) reward distributions in attempt to maximize the total reward (or equivalently, to minimize the total regret w.r.t the oracle policy). This problem has been extensively studied in the literature, especially since the seminal paper by Lai \& Robbins [115]. They introduced upper confidence bound (UCB) policies attaining a logarithmic regret. Another popular but fundamentally different low-regret policy is Thompson Sampling [100]. Being Bayesian in spirit, it is better suited for modeling complex online problems [71], which is why we adopt this method to build our recommender system. Since most work on multi-arm bandits has been theoretical in nature [185], their implementation in real-world scenarios such as content recommendations is still elusive [3]. This is because 
many of the assumptions made by traditional MAB are violated in practical applications. In particular, we envision the following challenges of implementing bandit recommendations in ecommerce:

Non-stationary rewards. Since behavioral signals in the data evolve with time, predictors of this type are characterized by non-stationarities in their reward sequences. Algorithms that deal with this issue include switching and restless bandits [35, 68, 131].

Multiple plays. The "top- $N$ ranking" problem dictates that multiple actions need to be taken in each round to ensure that all available item slots are filled. Problems of this type are addressed with ranked $[114,162,188]$ cascading $[113$, 120] and multiple-play bandits [71, 123, 197].

Variable availability of actions. In the real world, not all actions are available at each time instance. For example, this may happen when predictors find no correlations between items, or when items themselves are not accessible (e.g. out of stock). Bandit policies capable to accommodate such situations are known as sleeping bandits [99, 103, 184].

In this paper we propose a streaming (sequential) recommender system [74], which adapts existing MAB policies to the aforementioned scenarios. Motivated by the fact that many industrial recommenders tend to favor simple algorithmic approaches (e.g. best-seller lists) [152], our solution is essentially an ensemble learning scheme that uses Thompson Sampling to orchestrate the collection of elementary recommendation components. This allows content-based and behavioral signals in the data to effectively complement each other in response to user queries. The proposed model offers a number of advantages to a prospective vendor: 
- It allows to easily plug-in/out recommendation components of any type, without making changes to the main algorithm;

- Modeling components as arms helps to overcome the scalability limitation of the more common "items-as-arms" representation;

- Handling context can be shifted to the level of components, hence eliminating the need for contextual MAB policies.

- Elementary recommendation components are easily interpretable and hence allow for more transparent user interfaces that improve user's trust in the system.

The paper is organized as follows. The next section gives an overview of related variants of MAB policies, along with related studies on MAB-based recommender systems. Section Approach introduces our approach and details the proposed ensemble algorithm in pseudo-code. In Section Experiments we describe our experiments and present their results. We summarize and conclude our findings in Section Conclusions.

\section{Related Work}

The non-stationarity of arm rewards has been approached from different angles. In the presence of domain knowledge, the shape of the reward distribution may be known a priori. Bouneffouf and Féraud [25] show how to take advantage of this knowledge in UCB1 to tackle non-stationarity in music or interface recommendations. In many cases (including ours), the functional form of non-stationarity is not known. Therefore, automatic detection of abrupt or drifting changes in reward sequences has been proposed in several extensions to UCB [81, 68] and Thompson Sampling [131, 35] policies. These two types of behaviors (i.e. abrupt and drifting) correspond, respectively, 
to switching [68, 131] and restless [35] bandits. Garivier et al. [68] also show that any policy with a logarithmic regret for the stationary case is bound to $T / \log (T)$ regret in the presence of reward irregularities, where $T$ is the time horizon. In fact, the notion of dynamic regret has been introduced for drifting reward functions, which is measured w.r.t. a benchmark policy taking the best action for each round in isolation [184].

The multiple arm selection (top- $N$ ) problem appears to be highly relevant for recommender systems. The most straightforward solution, known as ranked bandits, assigns a separate bandit to each slot in the ranking [188, 162]. Lacerda [114] presents an interesting use case for ranked bandits that optimizes multi-objective ranking in recommender systems. More recently, cascading bandits have been proposed as a variant of a ranked bandit for modeling position bias in click data [113], and have been evaluated on movie recommendations [120].

Ranked bandits have been criticized for the underutilization of the available feedback, since it cannot be shared between individual bandits [123]. A better approach in this respect is the so-called multiple-play bandit, which can fully observe all incoming rewards. It is this approach we adopt in our work. A multiple-play bandit algorithm called EXP3.M, originally proposed in [197], has been assessed against two ranked bandit algorithms on movie and joke recommendations, demonstrating that EXP3.M indeed converges faster than the two ranked algorithms [123]. Likewise, Gopalan et al. [71] apply Thompson Sampling for the problem of top- $N$ arms selection and report a significant reduction in running time as compared to a decoupled bandit policy. They conclude that being Bayesian is advantageous for addressing complex bandit problems, and thus motivate our choice of policy.

Our problem setting, which resembles many other practical situations, assumes that certain actions may be unavailable at times. This has implications on the definition of regret, which is traditionally measured w.r.t. the best action in hindsight and 
is no longer applicable [103]. The idea then is to choose the best action among currently available actions ordered by their expected reward. Problem settings that exhibit such behavior have been coined sleeping bandits and studied in various contexts. For example, Kanade et al. [99] present a no-regret sleeping bandit algorithm for adversarial rewards, whereas Kleinberg et al. [103] analyze the regret for both adversarial and stochastic cases. A contextual sleeping bandit with stochastic rewards is presented in [184], wherein the author reproduces the result of [103] using contextual zooming.

Bandit algorithms are becoming one of the most promising approaches in the area of recommender systems [119]. Due to their exploratory nature, they are especially effective in attacking the cold-start problem (e.g. [128, 62, 36]). Referring to the e-commerce domain, Bernardi et al. [22] characterize this problem as the continuous cold-start, meaning that users and items remain "cold" for an extensive period of time. Moreover, existing users have the tendency to "cool down". This observation motivates the use of non-personalized i2i recommendations that we present in this work. We model bandit arms as predictors, which allows us to devise an ensemble method to tackle coldstart. This distinguishes our approach from others mentioned (e.g. [117, 206, 218, 114]), wherein arms represent single items. Whereas an item-as-arm model may be reasonable for few arms (as in [101, 117, 118, 142]), the magnitude of real-world data would likely lead to scalability issues [106].

Recent years have seen successful implementations of Thompson Sampling for recommendations of news articles [37, 192], movies [218], music [80], documents [26], learning courses [142], search queries [89], and dating partners [10]. Convincing attempts [101, 218] have been made to couple Thompson Sampling with popular matrix factorization techniques. Closest in spirit to our work is the paper by Tang et al. [192], who present an ensemble meta-bandit recommender system. Similar to our approach, they use a hyper-bandit ruled by non-contextual 
Thompson Sampling to adaptively explore/exploit base contextual bandits that select items to recommend. The same basic idea of using a bandit algorithm for prediction model selection in recommender systems has been conveyed by Felício et al. [62]. They experiment with UCB1 and $\epsilon$-greedy policies with arms modeled as clusters of users induced by matrix factorization. Our work differs mainly in how we model arms and attribute rewards. Contrary to some of the aforementioned recommenders that maximize the click-through rate (CTR) (e.g. [192, 37, 117, $123,114,3])$, we are interested in optimizing the number of sold units. In our experience, this e-commerce objective does not benefit from items that merely attract clicks, and therefore requires a different attribution model. We outline our approach in the next section.

\section{Approach}

\section{Problem Setting}

Following the reinforcement learning paradigm, we consider a typical e-commerce scenario as an interplay between:

- E-commerce environment comprised of a product catalog $I=\left\{i_{1}, \ldots, i_{|I|}\right\}$ and (possibly overlapping) user sessions, $S=\left(s_{1}, \ldots, s_{|S|}\right)$, each defined as $s=\left(q_{t_{x}}, \ldots, q_{t_{y}}, P_{\tau_{s}}\right)$, where query $q_{t} \in I$ represents a product page visit at time $t$, and $P_{\tau_{s}} \subseteq I$ is the final purchase order at time $\tau$ designating the end of the session (typically, $\left|P_{\tau_{s}}\right| \ll|I|$ ).

- Recommendation agent implemented as a multi-arm bandit with a set of actions $\mathcal{A}=\left\{a_{1}, \ldots, a_{|\mathcal{A}|}\right\}$ and a sequence of states $\Sigma=\left(\sigma_{1}, \ldots, \sigma_{|\Sigma|}\right)$. Each action represents a base recommendation function that maps a given query to some subset of the product catalog, i.e., if $Q$ is the set of all queries, then $a: Q \rightarrow \mathcal{P}(I \backslash Q)$, where $\mathcal{P}(\cdot)$ is the powerset function. 
We will only consider queries and purchases belonging to the same session and will, for ease of notation, omit session indices.

For each query $q_{t}$ with an associated $N$-sized recommendation list $L_{t}$, the agent takes at most $N$ available actions $A_{t}=$ $\left(a_{1}, \ldots, a_{\left|A_{t}\right|}\right)$, s.t. $\left|A_{t}\right| \leq N$ and $a_{i} \in \mathcal{A}$, according to some bandit policy $\pi$ so that the precision at time $t$ : prec@N(Lt $)=\left|L_{t} \cap P_{\tau}\right| / N$ is maximized. An action can appear more than once in $A_{t}$ and the recommender does not have access to $P_{\tau}$ at time $t$, since $t<\tau$.

Each display of an action's recommendation in $L_{t}$ results in a binary reward $r \in\{0,1\}$ after observing a purchase order at time $\tau$. We refer to each successful recommendation (i.e. the recommended item is eventually bought) corresponding to $r=1$ as a hit. The reward of an action that fails to generate a hit is zero $(r=0)$.

In case the same successful recommendation is given by different actions within a session, we face the problem of attribution, i.e. deciding which action should get credit for the sale [16]. We follow the equal multi-touch attribution strategy, according to which each action that generates a hit is equally rewarded. Alternative strategies include first-touch attribution, last-touch attribution, and fractional attribution (see [16] for details). Since we have no means of establishing which of the displayed recommendations are actually noticed by the user, we consider equal attribution as the safest choice.

For each action $a \in \mathcal{A}$, we maintain a tuple of running rewards $r_{a}$ and displays $n_{a}$, which represents the agent's current state of knowledge: $\sigma_{j}=\left(r_{a}, n_{a}\right)_{a \in \mathcal{A}}$. The agent moves to an updated state $\sigma_{j+1}$ after attributing rewards at time $\tau$. Figure 11 exemplifies the described mechanism.

The ultimate goal of the agent is to maximize the cumulative reward: $r_{T}=\sum_{t \leq T} r_{t}$, where $r_{t}$ is the sum of rewards obtained at time $t$. 


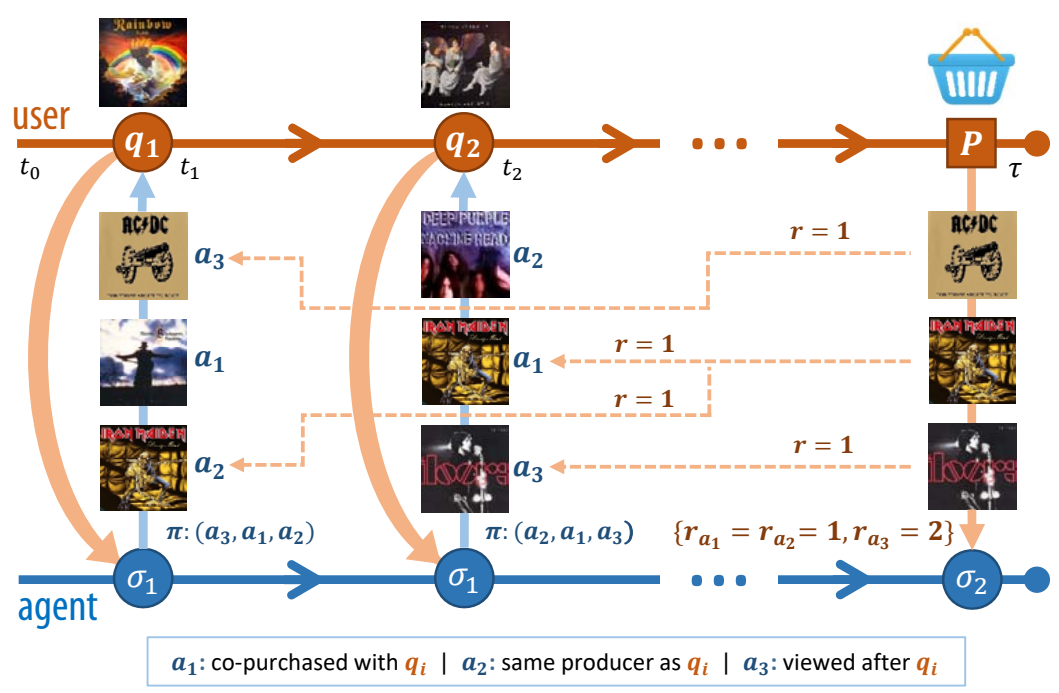

Figure 11: i2i session-based recommendations with explainable actions

\section{Base Recommenders}

As stated earlier, action set $\mathcal{A}$ is an ensemble of base recommendation functions or "components". ${ }^{17}$ These are simple contextual predictors, whose output is determined by the input query. Each component defines a graph $G_{a}((V, E), W)$, where $V$ is a set of vertices representing items, $E$ is a set of edges representing between-item associations (induced by $a$ ), and $W$ is a set of edge weights measuring the strength of these associations.

We distinguish between two types of components:

1. Attribute-based components connect items via their content features (i.e. metadata). We use elementary components, each representing a particular attribute of the product catalog. Edges of this type are undirected. For example, $N_{G_{\text {color }}}(v)$ retrieves the neighborhood of item $v$ containing all items of the same color(s) as $v$. Optionally,

\footnotetext{
${ }^{17}$ The terms 'actions', 'components', 'predictors', and 'recommendation functions' have the same meaning and are used interchangeably
} 
edge weights can be used to measure the number of attribute values that are matched.

2. Behavioral components connect items via events (e.g. purchases, clicks, etc.). The edge's weight in this case captures the frequency of event observations, i.e. the strength of the association. The edges can be directed, indicating that the event connecting two items is only meaningful in one direction. For example, we use the following four types of behavioral components:

- click-click (cc): the directed edge $\left(v, v^{\prime}\right) \in E\left[N_{G_{c c}}(v)\right]$ signifies that the click on item $v$ is followed by the click on item $v^{\prime}$.

- session-click (sc): the edge $\left\{v, v^{\prime}\right\} \in E\left[N_{G_{s c}}(v)\right]$ signifies that items $v$ and $v^{\prime}$ are both clicked within the same session.

- purchase-purchase (pp): the directed edge $\left(v, v^{\prime}\right) \in$ $E\left[N_{G_{p p}}(v)\right]$ signifies that the purchase of item $v$ is followed by the purchase of item $v^{\prime}$.

- customer-purchase (cp): the edge $\left\{v, v^{\prime}\right\} \in E\left[N_{G_{c p}}(v)\right]$ signifies that items $v$ and $v^{\prime}$ are both purchased by the same customer (not necessarily in the same session).

The two types of components correspond, respectively, to the terms content-based and collaborative filtering used in the recommender systems literature. A vendor can engineer components in many ways, for example by introducing personalization (as in 'customer-purchase'), campaigns, best-seller/special offer lists, or more complex feature combinations. Our choice of very granular predictors is dictated by the need for crossdomain applicability, since we want the recommender to operate well on various types of e-commerce data from complete cold-start. 
Dynamic Partitioning It is apparent that bandit arms constructed in this manner are prone to non-stationary behavior, since the size of the neighborhood $N_{G_{a}}(v)$ may differ significantly from query to query. The problem is particularly profound in behavioral components, as new edges are constantly added to the graph and the weights of existing edges are constantly updated from new observations. This may lead to situations when topperforming predictors (in the long run) get under-explored by the bandit algorithm because of their poor performance at an early stage.

A simple solution to this problem is to dynamically partition a component $a$ (i.e. its output) into several disjoint parts or "sub-components" based on the estimated precision of each candidate item contained in the query response $N_{G_{a}}(q)$. In other words, we cluster the response with the aim to obtain sub-components each having a distinct but homogeneous precision. Let $A_{a}$ denote a partition of component $a$ :

$A_{a}=\mathcal{P}(a)$, s.t. $\bigcap_{a^{\prime} \in A_{a}}=\varnothing, \bigcup_{a^{\prime} \in A_{a}}=a, \forall a \in \mathcal{A}$.

We can now expand the original action set to the set of subactions: $\mathcal{A}=\bigcup_{a} A_{a}$, where each sub-action $a^{\prime} \in A_{a}$ has a (relatively) fixed reward distribution.

To achieve this, we can use various proxies for precision depending on the type of component. For example, attributebased components can be partitioned based on the inverse document frequency $(\mathrm{IDF})^{18}$, as shown in Algorithm 9. The intuition behind it is that the more frequent the target attribute value is in other items, the more difficult it is to make the right recommendation. For behavioral components, we can directly use edge weights $w_{a}\left(v, v^{\prime}\right)$ corresponding to event observations as a proxy for precision. The obtained estimates are then thresholded to obtain the partition of the component's output. Because the event graphs are constantly evolving, partitioning is done dynamically upon each query response, and hence new

\footnotetext{
${ }^{18}$ Term frequency (TF) is omitted from calculations as the frequency of the attribute value in the same item is rarely greater than 1
} 


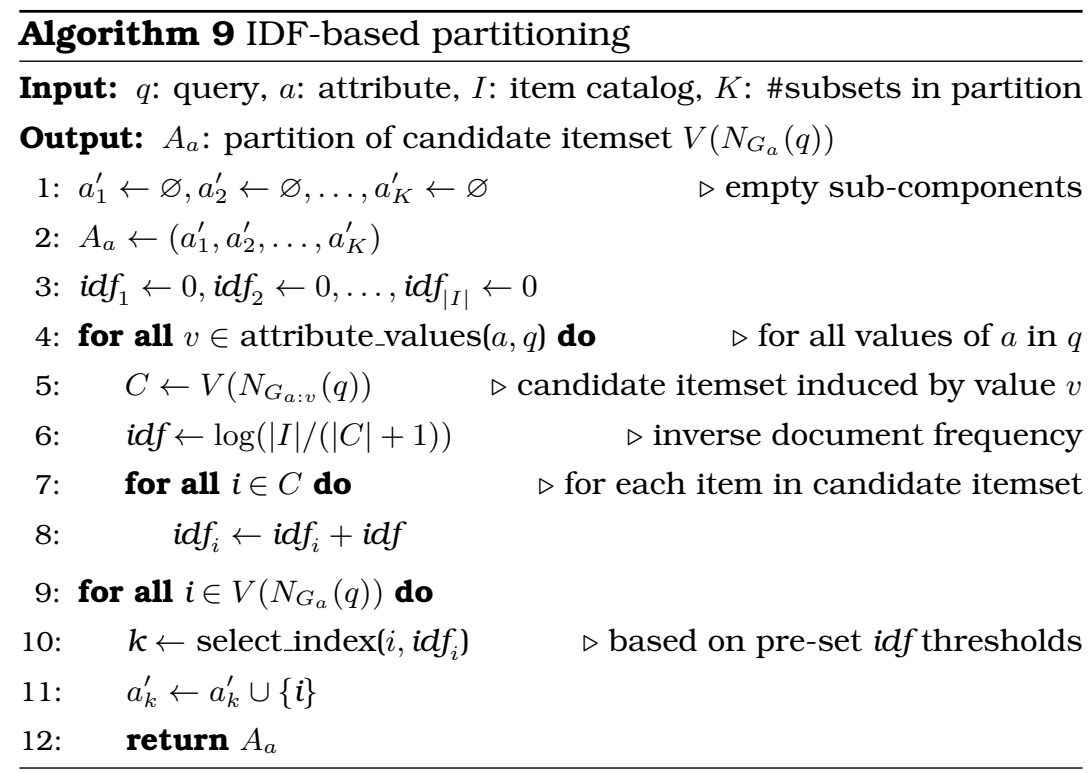

(more precise) sub-components may emerge with time.

\section{Ensemble Learning Agent}

Preliminaries Our agent extends beyond the classical multiarm bandit, since it needs to solve the top- $N$ selection problem with varied action availability. The availability of action $a$ for filling the $i$-th position in the top- $N$ list $L_{t}$ is subject to the following conditions:

1. $N_{G_{a}}\left(q_{t}\right) \neq \varnothing$, i.e. the response is non-empty

2. $N_{G_{a}}\left(q_{t}\right) \backslash L_{t}[1, \ldots, i-1] \neq \varnothing$, i.e. the response contains at least one item distinct from those already recommended.

Based on the above conditions and the policy $\pi$, the agent plays the best among currently available actions. This corresponds to picking one item from the action's sub-graph. The procedure is repeated for each position in $L_{t}$. The ensemble learner is therefore characterized as a sleeping bandit with multiple plays. Both recommendation steps (i.e. choosing an action 
and choosing an item) can be taken in either deterministic or probabilistic manner.

Because of the partitioning method presented earlier, we can apply any standard stochastic policy to our bandit ensemble framework, henceforth referred to as BEER (Bandit Ensemble for E-commerce Recommendations), where action rewards are assumed to be drawn i.i.d. from some fixed probability distributions. This also means that the selected action can now pick a random item from its sub-graph, which helps to diversify recommendations without loss in precision.

A well-studied family of deterministic MAB policies is based on the principle of optimism in the face of uncertainty. These policies select an action with the highest index corresponding to the upper confidence bound (UCB) for the action's expected reward, plus some padding function (e.g. [115, 68, 117]). They capture the intuition that the high bound is either due to the action being under-explored, or well-rewarded in expectation. Other policies balance between exploration and exploitation probabilistically. For example, the $\varepsilon$-greedy policy chooses a random action with probability $\varepsilon$ and the empirically optimal action with probability $1-\varepsilon$, whereas randomized probability matching chooses an action in proportion to its probability of being optimal. The latter approach is known as Thompson Sampling and has shown promising results in many practical applications, including top- $N$ action selection [71]. We now give an overview of this policy and then explain how to adapt it for the needs of our ensemble learning bandit (Algorithm 10).

Thompson Sampling Probability matching is a Bayesian heuristic, which models reward distribution using a parametric likelihood function $P(r \mid a, \theta)$, where $\theta=\left(\theta_{1}, \ldots, \theta_{|\mathcal{A}|}\right)$ is some unknown parameter vector. Our reward system implies a Bernoulli bandit, where parameters $\theta$ represents the probabilities of obtaining a binary reward. Given agent state $\sigma$ at the current step $t^{\prime}$ and some prior distribution over $\theta$, the posterior distribution is 
obtained using Bayes' rule:

$$
P(\theta \mid \sigma)=\frac{P(\sigma \mid \theta) P(\theta)}{P(\sigma)} \propto \prod_{t=1}^{t^{\prime}} P\left(r_{t} \mid a \in A_{t}, \theta\right) P(\theta)
$$

We then take an optimal action according to the probability matching principle:

$$
a^{*}=\underset{a}{\arg \max } \int \mathbb{1}\left[\mathbb{E}(r \mid a, \theta)=\max _{a^{\prime}} \mathbb{E}\left(r \mid a^{\prime}, \theta\right)\right] P(\theta \mid \sigma) d \theta
$$

Instead of computing the integral, Thompson Sampling draws random samples independently for each action $a$ from the respective posterior $P\left(\theta_{a} \mid \sigma\right)$ and chooses the action $a^{*}$ with the largest $\theta_{a}$. The prior is typically Beta-distributed, since it is conjugate to the Bernoulli distribution: $P\left(\theta_{a}\right) \sim \operatorname{Beta}(\alpha, \beta)$, where $\alpha=\alpha_{0}+r_{a}$ can represent the number of "successes", and $\beta=$ $\beta_{0}+n_{a}-r_{a}$ can represent the number of "failures".

The randomized nature of Thompson Sampling implies continuous exploration in situations when deterministic UCB policies get stuck in the same action over the extended period of time. This makes Thompson Sampling more robust in various practical scenarios, namely:

- Delayed feedback. Because of random sampling, the exploration phase does not suffer from the lack of regular posterior updates.

- Adding new actions. One can simply set the new action's prior to the default $\operatorname{Beta}(1,1)$ and let the ensemble algorithm continue its exploration naturally. In UCB, the exploration would be substituted by exploitation of the new action until its confidence bound becomes comparable to the bounds of other actions.

- Non-stationary actions. When a previously well-performing action starts failing, Thompson Sampling goes to the exploration phase faster than UCB. 
All the above scenarios are handled seamlessly without any algorithm parameterization, which is another advantage of Thompson Sampling. However, the existence of the Beta parameters allows to re-shape the reward distributions beforehand, as we discuss next.

Sampler Priming Parameters $\alpha>0$ and $\beta>0$ control the shape of Beta distribution with mean $\mu=\frac{\alpha}{\alpha+\beta}$ and variance $\sigma^{2}=$ $\frac{\alpha \beta}{(\alpha+\beta+1)(\alpha+\beta)^{2}}$. The prior parameters $\alpha_{0}$ and $\beta_{0}$ allow us to prime the sampler with some initial frequencies of successes and failures. At time $t_{0}, \alpha=\alpha_{0}$ and $\beta=\beta_{0}$. The de facto choice $\alpha_{0}=\beta_{0}=1$ corresponds to the uniform distribution (i.e. uninformative prior). We consider two practical situations when more informative prior parameters can be specified:

1. Recorded event data is available beforehand. This can happen when the recommender system is deployed on a pre-existing e-commerce site. In this case, parameters $\alpha$ and $\beta$ can be learned directly by running Thompson Sampling through the recorded data.

2. No recorded event data is available. This is the case of newly launched e-commerce sites, where only the product catalog is known. In this scenario, a vendor might attempt to prime attribute-based components based on the statistical properties of the product catalog.

In cases when the direct estimation of $\alpha$ and $\beta$ does not seem feasible (as in case 2), we might still make assumptions about the means of prior distributions based on some domain knowledge (e.g. product catalog). We can then reason about the variance through the following formula:

$$
\sigma^{2} \stackrel{\text { def }}{=} \frac{\mu(1-\mu)}{\nu}
$$

where $\nu>1$ is a parameter expressing our uncertainty about the estimated mean. This parameter is defined as $\nu \stackrel{\text { def }}{=} \alpha+\beta+1$. 
Choosing high values of $\nu$ (i.e. high certainty) results in more peaky curves, whereas lower values lead to flatter distributions. Knowing the estimates of $\mu$ and $\sigma^{2}$ allows us to compute $\alpha$ and $\beta$ as follows:

$$
\alpha=-\frac{\mu\left(\sigma^{2}+\mu^{2}-\mu\right)}{\sigma^{2}} \quad \beta=\frac{(\mu-1)\left(\sigma^{2}+\mu^{2}-\mu\right)}{\sigma^{2}}
$$

We provide further details about the estimation of $\mu$ and $\sigma^{2}$ for case 2 in Section Experiment 4: Priming the Sampler.

Algorithm Our bandit ensemble algorithm based on Thompson Sampling ("BEER[TS]") is summarized in the pseudo-code below.

\section{Algorithm 10 BEER[TS]}

Input: $\alpha_{0}^{(a)}, \beta_{0}^{(a)}:$ prior parameters $\forall a \in \mathcal{A} \quad \triangleright$ default: $\alpha_{0}=\beta_{0}=1$

Output: top- $N$ recommendations $L_{t}$, where $t$ is current time step

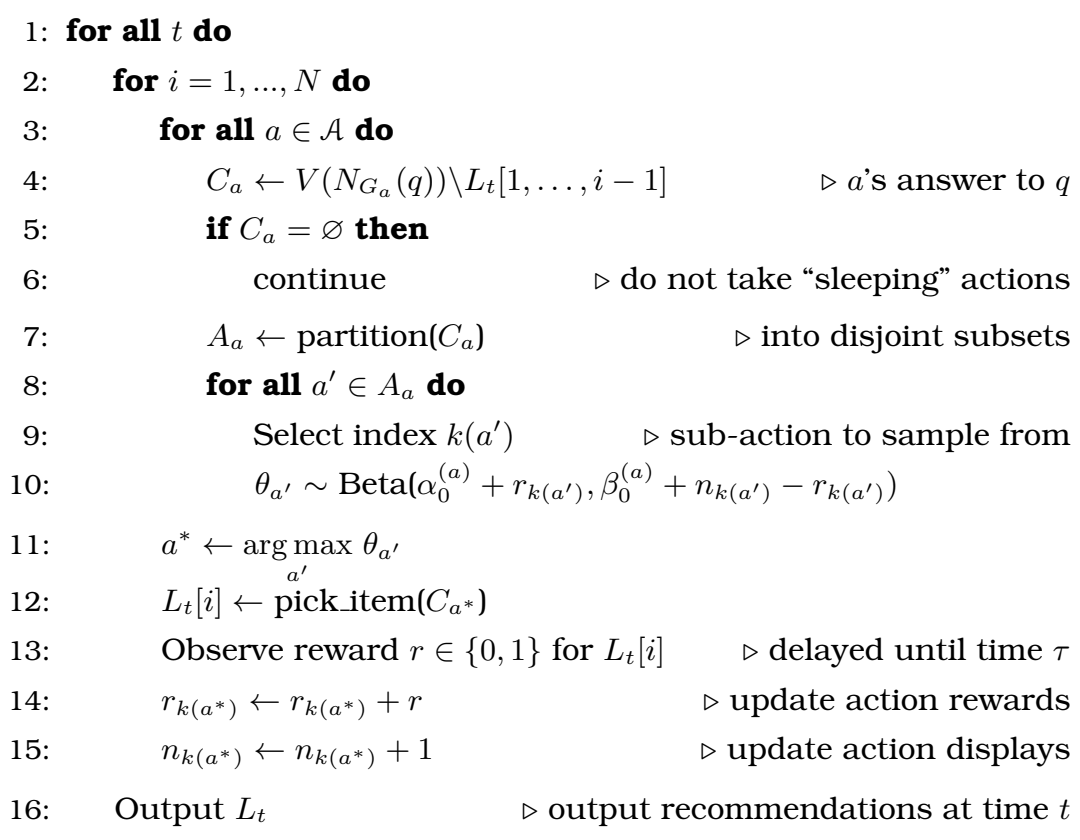

We note that it is easy to substitute TS with other MAB policies within the BEER framework. The experimental evaluation 
of different policies is given in Section Experiment 3: MAB Policies within BEER.

One step of Thompson Sampler takes constant time, and the time complexity increases linearly in the number of subcomponents involved in the learner.

\section{Experiments}

\section{Datasets and Evaluation Metrics}

We conduct our experiments using two proprietary e-commerce datasets ${ }^{19}$ from the books and fashion domains, and one public datase ${ }^{20}[20]$ with cross-domain merchandise. From these datasets, we select 500,000 events of timestamped session data for our experiments. We only consider sessions that culminate in a purchase order. The summary of the datasets is given in Table 10.

Table 10: Dataset summary

\begin{tabular}{llll}
\hline Dataset & Books & Fashion & Yoochoose \\
\hline \# Products & 91433 & 19731 & 52739 \\
\# Purchases & 134850 & 50096 & 101763 \\
\# Attributes & 45 & 51 & 2 \\
\# Sessions & 58465 & 21411 & 55600 \\
Mean session length & 8.6 events & 22.37 events & 9.25 events \\
\hline
\end{tabular}

In all our experiments, top- $N$ recommendations are evaluated at $N=5$. The accuracy of such recommendations is traditionally measured in terms of precision and recall. Precision shows how accurate the recommender system is in relation to the number of displays within a session, whereas recall evaluates its accuracy with respect to the number of purchases in a session. Let $h_{s}$ and $h_{s}^{\prime}$ denote, respectively, the number of total and distinct hits in session $s$. The above metrics can be defined

\footnotetext{
${ }^{19}$ Provided by Apptus Technologies: http: //www . apptus . com

${ }^{20}$ Provided by Yoochoose: http: //2015. recsyschallenge.com/
} 
as follows:

$$
\text { precision }=\frac{\sum_{s} h_{s}}{\sum_{s} n_{s}} \quad \text { recall }=\frac{\sum_{s} h_{s}^{\prime}}{\sum_{s}\left|P_{\tau_{s}}\right|}
$$

where $n_{s}$ is the number of displays in session $s$, and $P_{\tau_{s}}$ is the set of purchased items in session $s$.

In addition, we report the Normalized Discounted Cumulative Gain (NDCG), which is sensitive to the position at which a hit occurs. For a set of queries $Q, N D C G$ at rank $N$ is computed as follows:

$$
N D C G=\frac{1}{|Q|} \sum_{q=1}^{|Q|} \frac{D C G_{q}}{I D C G_{q}}
$$

where

$$
D C G_{q}=\sum_{i=1}^{N} \frac{r_{i}}{\log _{2}(i+1)}, \quad I D C G_{q}=\sum_{i=1}^{\min (|P|, N)} \frac{1}{\log _{2}(i+1)}
$$

All our experiments report the averages over 10 runs of the simulation.

\section{Experiment 1: Standard vs. Modified TS}

In our first experiment, we compare the modified Thompson Sampling policy adapted for non-stationary and "sleeping" actions (i.e. Algorithm 10) to the standard Thompson Sampling algorithm. The comparison table is given below.

Table 11 reveals that accounting for "sleeping" actions in Thompson Sampling is a necessary measure for our application, since it improves the accuracy on all three metrics and all datasets. The magnitude of the accuracy gain in each dataset is inversely proportional to the query response rate of each component of the ensemble. If frequently chosen components cannot answer many queries, the "sleeping" mode becomes more important. This claim is supported by our next experiment, where we report the query response rates for the datasets.

It can be seen that component partitioning is able to further boost the accuracy on all datasets in terms of precision and 
Table 11: Standard vs. modified Thompson Sampling

\begin{tabular}{llll}
\hline Agent & Recall & Precision & NDCG \\
\hline \multicolumn{4}{c}{ Books } \\
TS (SB+DP) & $\mathbf{0 . 0 5 8 1 ~} \uparrow 83.3 \%$ & $\mathbf{0 . 0 2 1 0} \uparrow 94.4 \%$ & $\mathbf{0 . 0 7 1 6} \uparrow 94.6 \%$ \\
TS (SB) & $0.0562 \uparrow 77.3 \%$ & $0.0202 \uparrow 87.0 \%$ & $0.0660 \uparrow 79.3 \%$ \\
TS & 0.0317 & 0.0108 & 0.0368 \\
\hline \multicolumn{4}{c}{ Fashion } \\
TS (SB+DP) & $0.1734 \uparrow 21.3 \%$ & $\mathbf{0 . 0 1 5 2} \uparrow 13.4 \%$ & $\mathbf{0 . 0 4 8 3} \uparrow 19.9 \%$ \\
TS (SB) & $\mathbf{0 . 1 7 6 0} \uparrow 23.2 \%$ & $0.0142 \uparrow 6.0 \%$ & $0.0427 \uparrow 6.0 \%$ \\
TS & 0.1429 & 0.0134 & 0.0403 \\
\hline \multicolumn{4}{c}{ Yoochoose } \\
TS (SB+DP) & $0.2973 \uparrow 10.5 \%$ & $\mathbf{0 . 0 4 1 9} \uparrow 10.8 \%$ & $\mathbf{0 . 1 3 9 1} \uparrow 21.4 \%$ \\
TS (SB) & $\mathbf{0 . 2 9 8 4} \uparrow 10.9 \%$ & $0.0409 \uparrow 8.2 \%$ & $0.1273 \uparrow 11.1 \%$ \\
TS & 0.2690 & 0.0378 & 0.1146 \\
\hline
\end{tabular}

$\mathrm{SB}=$ Sleeping Bandit, DP $=$ Dynamic Partitioning

NDCG ranking. In the Books dataset, it also improves recall. In the other two datasets, we observe the typical precision-recall trade-off, with recall suffering small losses yet remaining within comparable value ranges. This can be explained by the fact that sub-components have limited catalog coverage (as compared to an unpartitioned component), and hence can miss some items in $P_{\tau}$. However, they increase the likelihood of a relevant item being purchased by improving its exposure (precision) and ranking (NDCG) in recommendation lists. We also note that in this experiment we applied the same thresholds for component partitioning in all datasets, and hence it would be reasonable to expect higher scores with dataset-specific thresholding strategies. We leave this analysis for the future. In Table 11, TS (SB+DP) is equivalent to BEER[TS] from Algorithm 10.

Experiment 2: BEER(TS) vs. Baselines

We now compare our ensemble recommendation algorithm against two stand-alone recommenders, namely best sellers (BS) and those-who-bought-also-bought (TWBAB). Both predictors are 
based on item (co-)purchases, which is clearly the strongest behavioral signal in a non-personalized context. This makes these predictors highly competitive as baselines for our experiment. We evaluate two variants of BEER[TS] - with and without the aforementioned baselines as components of the ensemble. In this comparison, it is also worth looking at the coverage of queries by the recommenders. Table 12 summarizes the results of the experiment.

Table 12: Ensemble recommender vs. stand-alone baselines

\begin{tabular}{lllll}
\hline Agent & Recall & Precision & NDCG & Query coverage \\
\hline \multicolumn{4}{c}{ Books } \\
BEER[TS] incl. baselines & $\mathbf{0 . 0 5 8 9}$ & 0.0213 & $\mathbf{0 . 0 7 1 9}$ & $\mathbf{1 . 0}$ \\
BEER[TS] excl. baselines & 0.0551 & 0.0275 & 0.0655 & 0.7185 \\
TWBAB & 0.0324 & $\mathbf{0 . 0 3 6 5}$ & 0.0366 & 0.4164 \\
Best sellers & 0.0105 & 0.0085 & 0.0292 & $\mathbf{1 . 0}$ \\
\hline & \multicolumn{3}{c}{ Fashion } \\
BEER[TS] incl. baselines & $\mathbf{0 . 1 7 5 0}$ & $\mathbf{0 . 0 1 5 5}$ & $\mathbf{0 . 0 5 0 4}$ & $\mathbf{1 . 0}$ \\
BEER[TS] excl. baselines & 0.1704 & 0.0149 & 0.0474 & 0.9825 \\
TWBAB & 0.1185 & 0.0136 & 0.0277 & 0.7792 \\
Best sellers & 0.0490 & 0.0119 & 0.0374 & $\mathbf{1 . 0}$ \\
\hline & \multicolumn{2}{c}{ Yoochoose } & & \\
BEER[TS] incl. baselines & $\mathbf{0 . 2 9 8 5}$ & 0.0424 & $\mathbf{0 . 1 4 2 4}$ & $\mathbf{1 . 0}$ \\
BEER[TS] excl. baselines & 0.2953 & 0.0470 & 0.1376 & 0.8999 \\
TWBAB & 0.2311 & $\mathbf{0 . 0 5 1 9}$ & 0.1063 & 0.7286 \\
Best sellers & 0.1211 & 0.0234 & 0.0687 & $\mathbf{1 . 0}$ \\
\hline
\end{tabular}

TWBAB = those-who-bought-also-bought

Regardless of whether the two baselines are part of the ensemble or not, BEER[TS] vastly outperforms the stand-alone baselines in terms of recall and NDCG. It is also clear from Table 12 that TWBAB tends to be the most precise recommender (for Books and Yoochoose). However, this predictor has rather poor query coverage, which is especially evident in the Books dataset, with only $41.64 \%$ query response rate. The query coverage of BEER[TS] without baselines is also lower than in other datasets, which is why the Books dataset benefits more from 
the "sleeping" bandit configuration in the previous experiment (Table 11).

The best sellers baseline, on the other hand, is capable of answering every query (past the very first session), since its recommendations are not query-specific. Therefore, the inclusion of this baseline makes it a good fallback method for the ensemble learner. With the addition of TWBAB, the learner can benefit from TWBAB's high precision in cases when it gives an answer. Indeed, we observe that the inclusion of the two baselines to BEER[TS] results in better recommendation accuracy with an excellent query coverage.

\section{Experiment 3: MAB Policies within BEER}

As noted earlier, it is easy to substitute Thompson Sampling with other MAB policies within the BEER framework. In this experiment, we run several instances of our ensemble learner powered by various $\mathrm{MAB}$ policies. Apart from TS, we consider four variants of UCB algorithms, namely UCB1 [12], UCBTuned [12], UCB-Bayes [100], and KL-UCB [67], as well as the pre-tuned $\varepsilon$-greedy and MOSS [11] policies. The resulting scores for different policies are summarized in Table 13.

We observe that Thompson Sampling maintains its top rank for all metrics in all three datasets. In line with the findings of Gopalan et al. [71], we conclude that Thompson Sampling is the most effective bandit policy when it comes to solving complex practical online tasks, such as e-commerce recommendations. The strength of Bayesian policies is also evident from the fact that UCB-Bayes is the second best performer.

\section{Experiment 4: Priming the Sampler}

Having established the best policy for organising the ensemble components, we experiment with priming the Thompson Sampler with side information in attempt to give it an additional accuracy boost. As outlined in Section Sampler Priming, this 
Table 13: Ensemble learner with different MAB policies

\begin{tabular}{lccc}
\hline Agent & Recall & Precision & NDCG \\
\hline & Books & & \\
BEER[TS] & $\mathbf{0 . 0 5 8 9}$ & $\mathbf{0 . 0 2 1 3}$ & $\mathbf{0 . 0 7 1 9}$ \\
BEER[UCB-Bayes] & 0.0578 & 0.0210 & 0.0709 \\
BEER[UCB-Tuned] & 0.0568 & 0.0206 & 0.0694 \\
BEER[MOSS] & 0.0567 & 0.0205 & 0.0694 \\
BEER[UCB1] & 0.0526 & 0.0188 & 0.0642 \\
BEER[KL-UCB] & 0.0500 & 0.0195 & 0.0657 \\
BEER[E-greedy] & 0.0024 & 0.0117 & 0.0382 \\
\hline & Fashion & & \\
BEER[TS] & $\mathbf{0 . 1 7 5 0}$ & $\mathbf{0 . 0 1 5 5}$ & $\mathbf{0 . 0 5 0 4}$ \\
BEER[UCB-Bayes] & 0.1701 & 0.0147 & 0.0480 \\
BEER[E-greedy] & 0.1697 & 0.0151 & 0.0489 \\
BEER[MOSS] & 0.1656 & 0.0141 & 0.0463 \\
BEER[KL-UCB] & 0.1630 & 0.0149 & 0.0485 \\
BEER[UCB-Tuned] & 0.1602 & 0.0133 & 0.0437 \\
BEER[UCB1] & 0.1426 & 0.0114 & 0.0377 \\
\hline & Yoochoose & & \\
BEER[TS] & $\mathbf{0 . 2 9 8 4}$ & $\mathbf{0 . 0 4 2 4}$ & $\mathbf{0 . 1 4 2 4}$ \\
BEER[UCB-Bayes] & 0.2976 & 0.0422 & 0.1414 \\
BEER[UCB-Tuned] & 0.2975 & 0.0421 & 0.1412 \\
BEER[MOSS] & 0.2971 & 0.0422 & 0.1413 \\
BEER[UCB1] & 0.2955 & 0.0415 & 0.1394 \\
BEER[KL-UCB] & 0.1794 & 0.0287 & 0.0950 \\
BEER[E-greedy] & 0.1211 & 0.0234 & 0.0755 \\
\hline
\end{tabular}

information may come from two sources: a) pre-existing user sessions; and b) the product catalog.

Priming with pre-recorded event data The deployment of the recommender system on an existing website with some history of past user sessions makes it possible to prime the sampler in two ways. First, the behavioral components can be warmstarted with initial item associations from past events. This allows the sampler to take advantage of high-precision behavioral components from the very start. Second, after running the sampler through historical data we obtain the initial pos- 
terior distributions for each component, which allows us to assign informative priors to the Thompson Sampler. We evaluate both scenarios by running BEER[TS] through pre-recorded $100 \mathrm{~K}$ events preceding our test data for each dataset. In Table 14 we evaluate the effect of pre-training behavioral components and priming the component's Beta distributions separately.

Table 14: Priming the sampler with pre-recorded event data

\begin{tabular}{|c|c|c|c|}
\hline Agent & Recall & Precision & NDCG \\
\hline \multicolumn{4}{|c|}{ Books } \\
\hline BEER[TS]: pre-trained+primed & $0.0632 \uparrow 7.3 \%$ & $0.0231 \uparrow 8.5 \%$ & $0.0828 \uparrow 15.2 \%$ \\
\hline BEER[TS]: pre-trained & $\mathbf{0 . 0 6 3 3} \uparrow 7.5 \%$ & $0.0230 \uparrow 8.0 \%$ & $0.0822 \uparrow 14.3 \%$ \\
\hline BEER[TS]: cold-start & 0.0589 & 0.0213 & 0.0719 \\
\hline \multicolumn{4}{|c|}{ Fashion } \\
\hline BEER[TS]: pre-trained+primed & $0.1805 \uparrow 3.2 \%$ & $\mathbf{0 . 0 1 5 9} \uparrow 2.6 \%$ & $\mathbf{0 . 0 5 1 7} \uparrow 2.8 \%$ \\
\hline BEER[TS]: pre-trained & $0.1792 \uparrow 2.5 \%$ & $0.0157 \uparrow 1.3 \%$ & $0.0511 \uparrow 1.6 \%$ \\
\hline BEER[TS]: cold-start & 0.1749 & 0.0155 & 0.0503 \\
\hline \multicolumn{4}{|c|}{ Yoochoose } \\
\hline BEER[TS]: pre-trained+primed & $\mathbf{0 . 3 1 1 5} \uparrow 4.5 \%$ & $0.0434 \uparrow 3.1 \%$ & $0.1464 \uparrow 3.2 \%$ \\
\hline BEER[TS]: pre-trained & $0.3096 \uparrow 3.9 \%$ & $\mathbf{0 . 0 4 3 4} \uparrow 3.1 \%$ & $0.1466 \uparrow 3.4 \%$ \\
\hline BEER[TS]: cold-start & 0.2980 & 0.0421 & 0.1418 \\
\hline
\end{tabular}

It becomes clear that pre-training behavioral components has a noticeable effect on the accuracy of recommendations (even when the pre-recorded dataset is 5 times smaller than the test set). However, the effect of priming the Beta parameters is less evident. This especially holds for datasets with few components, such as Yoochoose.

Catalog-based priming A more challenging priming scenario is when the only available side information is the product catalog. Even though priming behavioral components in this situation is not feasible, we may still make informed guesses about the predictive ability of attribute-based components. One way of reasoning about their means is to make them inversely proportional to the average query response size. In other words, assuming that each query response from action $a$ contains one 
relevant item, the mean $\mu_{a}$ is equal to the probability of that item appearing in the top- 1 result. We can express it as follows:

$$
\mu_{a}=\frac{1}{\left|V\left(G_{a}\right)\right|} \sum_{q \in V\left(G_{a}\right)} \frac{1}{\left|N_{G_{a}}(q)\right|}
$$

The calculation of the variance is done via Equation (26), where we need to set the uncertainty parameter $\nu$ according to our confidence in the estimation of $\mu_{a}$. We envisage several possibilities:

1. Uniform $\nu$. For the standard Thompson Sampling with priors $\alpha=1, \beta=1$, we have: $\nu=\alpha+\beta+1=3$. This corresponds to the default variance of $1 / 12$.

2. Component-specific $\nu$ depending on the component's query coverage. For example, $\nu_{a}=\sqrt{\left|V\left(G_{a}\right)\right|}+1$. Intuitively, the more items (i.e. queries) have contributed to estimating the means, the more reliable that estimate is. Plugging this $\nu_{a}$ to Equation (26) will result in variances reflecting our degree of uncertainty in the primed means.

Another factor worth considering is posterior scaling. In classical Thompson Sampling, all actions start off with uniform priors with $\mu=0.5$. In most recommendation scenarios, however, it is reasonable to assume that the true Beta means will be much closer to 0 . This can be seen in Table 12, where the precision of the best stand-alone predictor (TWBAB) is at best 0.0519 . Therefore, we may aid convergence by setting the initial means to lower values, such as 0.05 . In case the distributions are not uniform but primed, we can scale the primed means to the interval $[0,0.05]$.

We now evaluate the catalog-based priming of attribute-based components based on Equation (30) and the two aforementioned variance estimation strategies. Behavioral components cannot be primed in this case and hence maintain their uniform distributions, but are scaled as explained above. This time 
we exclude the Yoochoose dataset from the analysis, since it only has 2 attribute-based components. The results for the two other proprietary datasets (having 45 and 51 attribute-based components) are presented in Table 15.

Table 15: Catalog-based priming

\begin{tabular}{|c|c|c|c|}
\hline Agent & Recall & Precision & NDCG \\
\hline \multicolumn{4}{|l|}{ Books } \\
\hline BEER[TS]: catalog-primed, $\nu_{a}=\sqrt{\left|V\left(G_{a}\right)\right|}+1$ & 0.0592 & 0.0214 & 0.0721 \\
\hline BEER[TS]: catalog-primed, $\nu_{a}=3$ & 0.0587 & 0.0213 & 0.0718 \\
\hline BEER[TS]: cold-start & 0.0587 & 0.0212 & 0.0716 \\
\hline \multicolumn{4}{|l|}{ Fashion } \\
\hline BEER[TS]: catalog-primed, $\nu_{a}=\sqrt{\left|V\left(G_{a}\right)\right|}+1$ & 0.1761 & 0.0156 & 0.0506 \\
\hline BEER[TS]: catalog-primed, $\nu_{a}=3$ & 0.1752 & 0.0157 & 0.0508 \\
\hline BEER[TS]: cold-start & 0.1750 & 0.0155 & 0.0503 \\
\hline
\end{tabular}

Our results indicate that priming the sampler in the absence of any historical event data is indeed feasible, but the additional accuracy gain obtained in our experiments is negligible (below 1\%). While other approaches for the estimation of primed means might yield slightly better results, we conclude that adjusting prior parameters is not crucial for the convergence of Thompson Sampling, as the algorithm learns very fast anyway. However, what helps to warm-start the algorithm, as is shown in Table 14, is pre-training behavioral components using past event data.

\section{Conclusions}

\section{Contribution Summary}

E-commerce is one of the most important and challenging application domains of recommender systems, where recommendations must be adaptively computed for each new product page visit. Such an interactive user interface makes it easier for a user of an e-commerce service to find the product(s) of interest as $\mathrm{s} / \mathrm{he}$ explores the product catalog. 
In this paper, we have proposed an item-to-item ensemble recommendation algorithm with Thompson Sampling at its core. This algorithm was designed with a commercial application in focus, making it simple for a potential vendor to introduce recommendation functionalities to new or existing ecommerce platforms. The recommendation components of the ensemble can be constructed automatically from the existing item attributes or user-item interaction types (collaborative signals). At the same time, the system allows vendors to effortlessly integrate handcrafted components into the ensemble, which are immediately picked up and explored by the Thompson Sampler. Such a component-based architecture is scalable, since the number of bandit arms does not depend on the number of items. Furthermore, it allows system developers to deliver semantically transparent user interfaces [108], where each recommendation is accompanied with a clear explanation of its source (e.g. behavioral or attribute-based, see Figure 11). An integral part of our recommendation bandit is its adaptation to realistic situations with "sleeping" actions and non-stationary reward sequences.

In a series of experiments on real e-commerce session data, we show that the above adaptations are crucial for the algorithm's operation (with accuracy gains ranging from $83 \%$ to $94 \%$ for the Books dataset). We further evaluate the strength of the ensemble approach, achieving far superior results in comparison to strong stand-alone baseline recommenders, namely best-sellers and those-who-bought-also-bought. As we have observed, adding these baselines to the ensemble does not only improve its accuracy, but also attains 100\% query coverage. Our comparative study convinces us that Thompson Sampling is empirically the best MAB policy for the BEER framework. We further show that Thompson Sampling handles cold-start gracefully out-of-the-box, as it does not seem to benefit much from Beta parameter priming. This property together with TS's robustness to observation delays makes it a very attractive me- 
thod for attacking the recommendation problem using reinforcement learning.

\section{Future Work}

The proposed ensemble algorithm is as good as the components that it is comprised of. It is therefore worth exploring better strategies for constructing base predictors for the ensemble. When it comes to behavioral signals, we expect that the provision of personalized components based on existing collaborative filtering techniques would be advantageous for the ensemble, provided that user profile data is available. This way we can take advantage of both short-term and long-term user preferences when computing recommendations. With regard to attribute-based components, the next research direction is to develop methods for the automatic composition of high-precision attribute combinations as potential components of the ensemble. In addition, it is worth considering components built from item descriptions and/or images. Finally, it would be interesting to follow the recent success of deep reinforcement learning in playing video games in attempt to adapt it for the sequential recommendation problem in e-commerce. 


\title{
PAPER III
}

Paraschakis D.

"Towards an Ethical Recommendation Framework"

\begin{abstract}
The goal of our study is to provide a holistic view on various ethical challenges that complicate the design of recommender systems (RS). The articulated ethical recommendation framework maps RS design stages to the corresponding ethical concerns, and further down to known solutions and the proposed user-adjustable controls. The need for such a framework is dictated by the apparent lack of research in this particular direction and the severity of consequences stemming from the neglect of the code of ethics in recommendations. This framework aims to aid RS practitioners in staying ethically alert while taking morally charged design decisions. At the same time, it would give users the desired control over the sensitive moral aspects of recommendations via the proposed "ethical toolbox". The idea is embraced by the participants of our feasibility study.
\end{abstract}

Published in Proceedings of the 11th International Conference on Research Challenges in Information Science (RCIS), IEEE, 2017. Best paper award. 


\section{Introduction}

In the era of Big Data, when the vast information overload makes it challenging for consumers to locate the products or services they need, recommender systems (RS) offer a helping hand in filtering the data. The notion of recommendations is built on real-life experiences and therefore perceived by humans as something inherently positive. User studies indeed show that the mere fact of labelling items as "recommendations" increases their chances of being consumed [44, 105]. Whenever this fact is exploited for reasons beyond serving user needs, an ethical problem arises. Formally, ethics can be defined as "the study of morality" [194], where morality in turn can be defined as "the system whose purpose is to prevent harm and evils" [69]. As we show later, in the context of recommender systems these "evils" may include privacy intrusion, identity theft, behavior manipulation, discrimination, offensive / hazardous content, misleading information, etc. Thus, we can define recommendation ethics as the study of the moral system of norms for serving recommendations of products and services to end users in the cyberspace. This system must account for moral implications stemming from both the act of recommending per se, and the enabling technologies involved. A holistic view on recommendation ethics is currently lacking in the field of RS, despite the massive research that it attracts nowadays. According to the recent study by Tang \& Winoto [193], there exist only two publications ([169, 186]) that specifically address the problem of ethical recommendations. Still, they only focus on particular problems in particular applications. Concerns over the lack of holistic approach have also been brought up by Friedman et al. [65] who studied the privacy aspects of recommender systems - the subject that has drawn the most attention in the ethical discourse around the practices of big data. Another recent paper by Koene et al. [107] points to the striking research imbalance in the area of personalized RS. The 
authors note that the strong emphasis on the commercial success of recommender systems contrasted with the considerable neglect of moral values, has a potential risk of a future public backlash against this research area. Our study is also in line with the rapidly growing attention to Fairness, Accountability, and Transparency (FAT) in machine learning.

Recommendation ethics is a multifaceted problem, which relates to several interconnected topics that we broadly group into the ethics of data manipulation (privacy, anonymity, censorship issues), algorithm design (algorithmic biases, behavior manipulation, discrimination issues), and experimentation (fairness, awareness, informed consent issues). In Section Theoretical background: ethical challenges, we touch upon all these topics in relation to recommender systems. In Section Summary as a framework, we outline an ethical recommendation framework that serves two purposes: a) it provides a roadmap for an ethics-inspired design of RS; b) it proposes a toolbox for manual tuning of morally-sensitive components of RS. We evaluate our proposal in Section Feasibility Study by analyzing the results of the conducted survey. Section Conclusion concludes our work.

\section{Theoretical background: ethical challenges}

This section discusses various ethical challenges around recommender systems that have been identified in the related literature.

\section{Data collection and filtering}

Both types of personalized information filtering used in recommender systems - collaborative and content-based - require the construction of a user profile, expressed in either item attributes (metadata), or user interactions (ratings, purchases, etc). Unique user profiles make it possible to tailor recommended items to user's individual preferences.

Collecting behavioral data is often done in the absence of in- 
formed consent. Public surveys point to the great demand for "do not track" tools that would help users gain control over the data collection process [59]. Often, a notice about data logging is hidden inside the Terms of Service (ToS) [107], which users are expected to read and agree with. However, the well-familiar line "I have read and agree to the terms" has been coined as "the biggest lie on the Internet" [146] due to the fact that people either do not read or do not understand these lengthy and legalese-heavy notices. Even if they did, they would still have to spend 40 minutes per day on average for doing so [130]. A number of initiatives ${ }^{21}$ have been launched to improve the current situation by standardizing ToS, introducing trust marks or centralized monitoring. In Europe, the EU Cookie Law attempts to strike a compromise between brevity and informativeness by enforcing minimalist pop-up banners for acquiring user consent to store cookies. Sadly, both approaches fall victim of a "click to close" attitude from internet users [107]. It is worth noting, however, that the legislation pertaining to data collection, use, and disclosure has become more comprehensive in recent years with the introduction of the U.S. Consumer Privacy Bill of Rights and the European Privacy Directive [65].

To improve the accuracy of recommendations, user profiles can further be enriched by gathering additional data from external sources. This can be achieved by means of tracking cookies [170], linked open data [82], social networks [47] and even information brokers who collect and resell user activity data [146]. Whereas exceptionally detailed user profiles have positive effect on personalization, their leakage and misuse can put user's privacy at risk. Friedman et al. [65] distinguish three major sources of privacy breaches in recommender systems: a) the recommendation engine itself (and the people behind it), when its operation does not live up to users' individual expectations of privacy; b) RS users, who may attempt an inference

\footnotetext{
${ }^{21}$ http://www. commonterms.net, http://tosback.org, http:// www.truste.com, https://wiki.mozilla.org/Privacy_Icons
} 
attack on each other's profiles by observing RS outputs; and c) external adversaries (e.g. hackers), who may attempt to reidentify subjects in anonymized data (see more in Section Data publishing and anonymization).

When fallen into the wrong hands, behavioral profiles built for serving personalized recommendations may as well be utilized for malicious purposes, such as phishing or social engineering [107]. Moreover, disclosed user profiles may reveal sensitive private information either directly (e.g. health records) or indirectly (e.g. by inference from movie ratings). Profile injection is one of the possible attacks, which uses fake profiles to promote or demote recommendations of certain items [32]. For example, it can be used to suppress recommendations of competitor's products by giving them low ratings from a fake profile. To tackle this problem, some websites (e.g. Booking.com) enforce user identity verification as a prerequisite for leaving feedback. The emergence of recommendations-as-a-service (RaaS) in the past few years has also raised privacy concerns due to the exposure of user profiles to a third party [65].

A number of privacy preserving collaborative filtering (PPCF) techniques have been proposed in RS literature (e.g. [118, 157, 214]) to protect user profiles from misuse. These solutions can be broadly classified into architectural (protocols, certificates), algorithmic (cryptography, differential privacy, pseudonymity, obfuscation), and normative (policies, regulations) [65]. The major challenge in such privacy-enhanced RS is to preserve the recommendation accuracy at an acceptable level. A comprehensive overview of privacy aspects of RS can be found in [65]. It is therefore crucial that RS developers become aware of potential privacy implications and take the necessary precautions during the initial data gathering / user profiling stage.

Apart from employing privacy-enhancing technology (PET), recommender systems can benefit from graphical user interfaces (GUIs) that enable users to manage their profile data by defining interests, cleaning up browsing history, and so on (e.g. 
see Amazon's "manage history" tool). Bakalov et al. [13] show that this has a positive effect on RS usefulness and usability, as well as on the overall user satisfaction. The reason is that users have different perceptions of privacy and different attitudes towards the associated protective measures [21]. To this end, user-tailored privacy decision support for recommender systems has received considerable attention in the literature [105, 104, 207]. Despite the provided ability to manually withhold items from one's profile history, there is a danger that these items may still be inferred from other information exposed to a recommender system [65]. Therefore we advocate the use of PET-based and GUI-based solutions in combination as a complex protective measure.

The final stage of information filtering in a RS delivers relevant items to a user. With very few exceptions ( [169, 186, 193]), existing systems do not employ any ethical filtering of their output. This raises an issue of content censorship with all its consequent implications. As well noted in [193], what is algorithmically appropriate is not necessarily ethically appropriate. The serendipitous nature of a collaborative RS adds an extra challenge due to its tendency to produce unexpected results. Apart from the obvious need for parental control, one might think of morally troubling examples of recommending meat-based products to a vegetarian, gay-intolerant movies to a homosexual, alcohol drinks to a religious Muslim, or tobacco products to a person who struggles to quit smoking. Should the recommender system be held accountable for serving offensive or even hazardous content? How can it mitigate the risk of moral harm? The ethical appropriateness of candidate items can be established by mapping potentially harmful elements in media content (drug use, nudity, etc.) to a user's persona (gender, age, religion etc.), as explained in [193]. The authors suggest that moral values are incorporated into system requirements, with users having control over the filtering process. We add that moral values can be treated as a special type of con- 
text, which can be used in pre-, post-, or in-filtering stages of recommendations. An attempt to build a fully-automated ethical RS is made in [186], where the human intervention is not required. Yet, the reliability of such a system largely depends on user-specified demographic data, which can often be omitted or falsified by users due to privacy concerns. Besides, applying an automatic censorship filter in the complete absence of user control (and consent) would also be morally problematic. Facebook's profanity filter for blocking offensive words serves as a good example of a user-adjustable censoring tool.

\section{Data publishing and anonymization}

The active research on recommender systems would hardly be possible without the availability of publicly released datasets from online services such as Netflix, MovieLens, or LastFM. Annual RecSys challenges and contests such as the Netflix Prize propel research and help businesses improve their recommendation engines by means of crowdsourcing RS algorithms from a large academic community. Because a public dataset contains private data [132], releasing a myriad of user records bears serious moral responsibility. The risks of disclosing a user profile were mentioned in the previous section. Here, we continue this discussion from the perspective of privacy preserving data publishing (PPDP).

For several decades now, anonymization has been used to disguise personally identifiable information (PII) in public datasets by employing such techniques as generalization, suppression, perturbation, or anatomization [212]. Barocas et al. [18] define anonymity as the state of complete unreachability, whose absence would pose a threat to one's ethical and political freedoms. The naive assumption that such anonymity can be achieved by disguising explicit PII such as names or phone numbers has long ceased to hold due to the existence of quasi-identifiers. When combined, these seemingly harmless fields are capable of 
uniquely identifying a user (e.g. $87.5 \%$ of U.S. residents can be identified via a $\langle Z I P$ code, birth date, gender $\rangle$ combination [189]). However, user demographics is a valuable input for many recommenders, and a too aggressive anonymization may impact the usability of a dataset (especially for a content-based RS). This challenging trade-off between privacy and utility lies in the core of PPDP. Taking an online pharmacy dataset as an example, its public release might go either of the two extreme ways, both leading to a highly undesirable outcome:

1) heavy anonymization $\rightarrow$ inaccurate recommendations $\rightarrow$ purchase of incompatible medications;

2) poor anonymization $\rightarrow$ re-identification of subjects $\rightarrow$ disclosure of sensitive health records.

Data owners are therefore encouraged to perform a privacy calculus [105] before deciding on whether and how a public release should be administered. This is done by "multiplying" the risk of disclosure by the sensitivity of the data [147], weighted against the anticipated benefits. Such privacy calculus can potentially be automated, as explained in [105]. If data sensitivity is domain-dependent, should there be regulations dictating how to treat data from a specific domain before the release? Should there be different levels of domain-specific data access for different types of public (researchers, students, data brokers, advertisers, etc.)? Corporate ethics sometimes result in semi-public releases, when the dataset's accessibility by external analysts is bounded by the physical organizational boundaries. Notably, anonymization may become a necessity even on platforms with a closed recommender system [82], which operates on a privately maintained dataset. This is done to protect user identities when the data is shared between different departments of a large organization [147].

Notably, various anonymization techniques aimed at protecting privacy in public data are ethically problematic in themselves. This is because obfuscated user profiles can be interpreted as "invalid data", which may potentially lead to incorrect 
inferences about individuals (even if the recommendation accuracy remains high). This may have negative psychological and even legal consequences [65].

The possibility of aggregating data from external sources unlocks the door to easy re-identification even in the presence of substantial anonymization. This can be done through a linking attack [124], which matches records in different databases via quasi-identifiers. This attack was effectively used to deanonymize the Netflix dataset (containing 500,000 users) only two years after its public release [139]. As a result, the planned Netflix prize sequel was discontinued due to the filed lawsuit [107]. It was shown in [139] that with some minimal and imprecise outside information about the user (a couple of public ratings from IMDb and their approximate dates), it was possible to identify him/her in the Netflix dataset with high probability. Apparently, disclosed movie ratings can be used to infer sensitive facts about a person, such as their political/religious views, or sexual status. Moreover, revealing movie rating history may have implications on user's future privacy [139]. Naturally, this applies to other types of preference data too (music tastes, Facebook "likes", etc.). The risk of privacy breaches is further exacerbated because of the accretion problem [147], which refers to the snowballing effect of chained linking attacks on the increasing number of databases. Linking large chunks of external data has become easier than ever thanks to the rise of big data, semantic web, and social networks. The case of de-anonymizing the completely PII-less Twitter graph by intersecting it with the social graph of Flickr serves as an instructive example [140]. Ethical concerns about de-anonymization of data might explain the limited number of public datasets available for RS research.

Figure 12 depicts moral bonds between the three main stakeholders in a data publishing cycle, namely data collector, data publisher, and data recipient. These bonds reflect the moral responsibility of stakeholders towards each other, as well as the 


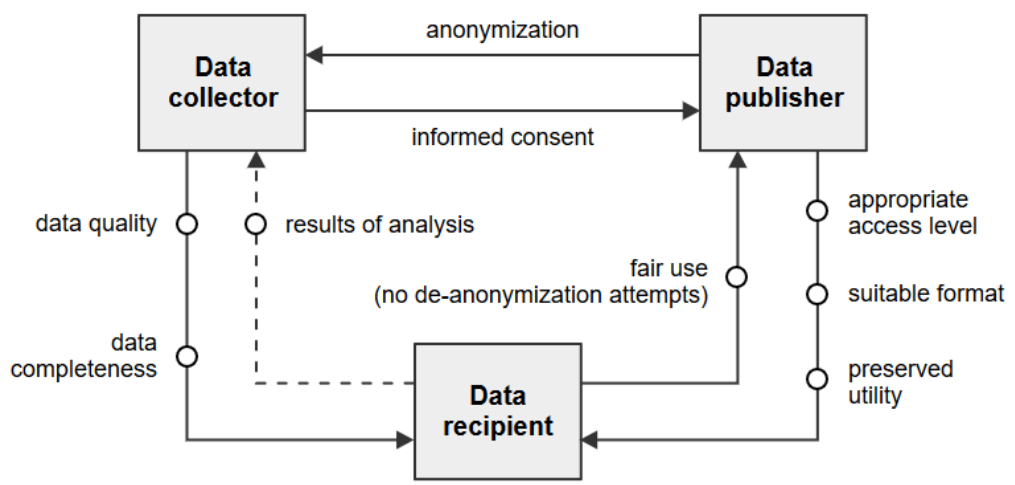

Figure 12: Moral bonds between stakeholders in the data publishing process

data subjects involved. The responsibility of the data collector is to ensure that the data sample is representative of the population, clean, and contains the necessary attributes. The consent is then given to the data publisher to proceed with the release. In return, the data publisher guarantees anonymity and privacy protection of data subjects. In front of the data recipient, the publisher is responsible for making the dataset accessible (in compliance with the active policies), properly formatted, and usable for analysis. The data recipient is morally entitled to the fair treatment of data and - in some cases and upon agreement - communicating the results of the analysis back to the data collector/owner. We note that this is just an idealized framework, whose practical implementation is currently confronted with challenges of technological or legislative nature.

While privacy models are being developed by computer scientists to aid PPDP (differential privacy [57], $k$-anonymity [190], l-diversity [124], etc.), lawmakers fight their own battle to regulate data publishing and catch up with the ethical realities of big data analytics. At the very least, legislators must acknowledge that the traditional anonymization paradigm based on the removal of PII is nothing more than a half-measure [147]. We refer the reader to [147] for an in-depth discussion of the regu- 
latory challenges concerning PPDP.

Algorithmic opacity, biases, and behavior manipulation

A typical recommender system operates as a black box, with its output being the only part that is visible to a user. Whereas certain input signals can also be observed (e.g. product attributes, personal purchase record, public ratings), aggregating and processing them into recommendations is done completely behind the scenes (Figure 13).

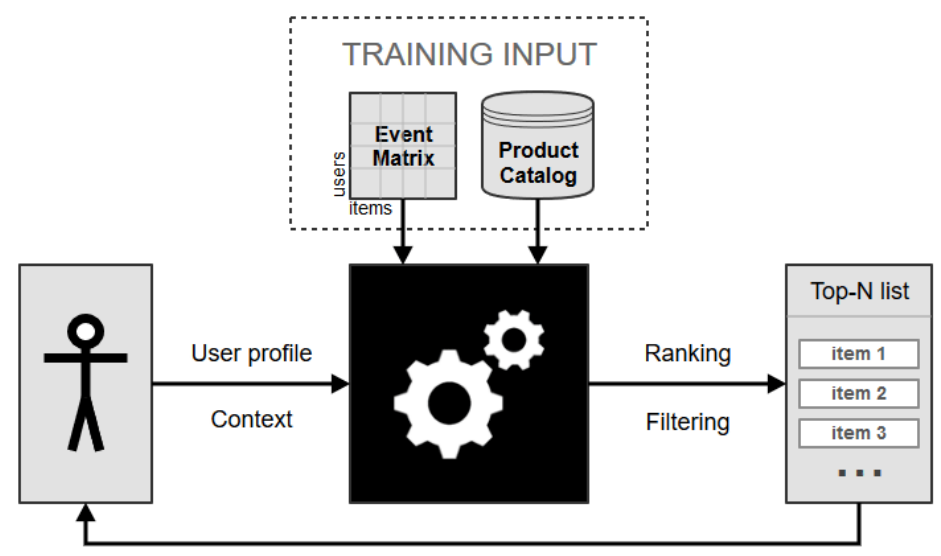

Figure 13: High-level view of a personalized recommender system

What are the ethical implications of not knowing the algorithm that rules recommendations? Apparently, this opacity makes it hard to reason about the political, economic, and cultural agendas behind these suggestions [154]. It can also hide the evidence of algorithmic discrimination [176] and behavior manipulation [107]. As Pasquale [154] notes, "faulty data, invalid assumptions, and defective models can't be corrected when they are hidden". This is why algorthmic opacity is viewed as a serious ethical issue in data science. Should users be allowed to look inside the black box? The survey by Herlocker et al. [84] reveals that $86 \%$ of users would like to see an explanation interface as an integral part of the recommendation service. The simplest example is Amazon's "customers who bought this 
item also bought..." recommendation interface. Automatic explanations can provide transparency into how and why a recommendation was generated, and hence improve users' trust in the system [84, 195]. Especially when a recommender system gives bad advice, users deserve an explanation and a possibility to correct the system's reasoning (this property is known as "scrutability") [195]. One way of aiding transparency is to report the system's confidence in generated recommendations.

Whereas explanations offer a way to unbox a recommendation engine, they also have a number of ethical implications. This practice often comes in conflict with corporate ethics that may forbid the disclosure of an algorithm for fear of losing competitive advantage or making it vulnerable to intentional manipulation [52]. The challenge in this case is to find other ways to justify recommendations without having to open the black box. Some examples of such justifications are given in [84]. Other alternatives proposed in the literature include routine algorithm audits by third parties [176], and reverse engineering methods [52]. Perhaps less obvious, there also exists a privacy threat that exploits the fact that explanations reveal connections between items. Combined with some side information, these connections would allow an adversary to identify a specific person and reveal their personal details [163]. Finally, the provision of transparency is impeded because of the interpretability problem [34]. Even if an algorithm is not kept in secrecy, explaining it to a user may hardly be possible because of its inherent mathematical complexity. For example, some recommendation methods are based on latent semantic analysis, where the distinguishing factors do not have a clear interpretation [195]. The problem is further exacerbated by the "course of dimensionality", which makes it impossible for a reasoning human to grasp the extreme multitude of features involved in computation [34]. Besides, unveiling the algorithm in this case would not be sufficient, since predicting its behavior without knowing the specific feature values is virtually impossible [176]. Finally, some 
of the most successful recommender systems are implemented as hybrid and ensemble models, combining several algorithms and data sources. Comprehending the reasoning behind such complex systems may be very hard even for trained computer scientists.

In their interaction with RS, how can users be certain that their interests are respected and prioritized? What if the recommendation output is severely biased and their behavior is being intentionally manipulated to meet certain business objectives? Documented examples of algorithmic bias include recommending lower paying jobs to female candidates, or higher priced flights to MacBook owners [168]. As Ross [172] notes, there is a big difference between using a dataset for describing a population and using it for manipulating the behavior of that population. For instance, this can happen when a recommender system is exploited to push expiring, overstock, delisted, and other first-priority items that do not necessarily answer the needs of a user. It may be tempting for a business to disguise such items under misleading category names such as "best sellers" in order to boost the utilization of any unwanted inventory. In a similar way, privileged recommendation categories (e.g. "top hotels") can be easily populated with paid listings that may not correlate well with the category label.

Another type of bias that can be encountered in recommender systems is price discrimination. This enables a RS to adjust prices on the fly based on the user's willingness-to-pay (WTP) value, defined as the maximum amount of money s/he is expected to pay for a given product. It has already been shown that travel sites Cheaptickets and Orbitz intentionally raise hotel prices for visitors who do not have an account with them [79]. Another example is Amazon's short-lived experiment on selling DVDs at different prices to different users, which provoked a furious reaction showing the profound moral impact of price discrimination [200]. Nevertheless, this did not stop researchers from proposing recommendation models with per- 
sonalized pricing (e.g. [98, 129, 217]). Unfortunately, the ethical feasibility of such systems remains poorly addressed and hence questionable, especially considering that price discrimination is illegal in some jurisdictions. Even when it is not, this serves as a good example of when legal does not equate to ethical. An even worse case can be made when a discrimination (of any type) is based on morally sensitive attributes (gender, race, religion, etc.) that are either directly provided by a user, or inferred from other information available to a recommender system (as discussed earlier). For example, when a person is to be recommended for a loan, gender-discriminatory decisions may introduce dangerous "false negatives" that may potentially lead to lawsuits [202]. Such systems can (and should) benefit from specialized techniques for discrimination-aware data mining [61].

Personalized news recommenders, to a large extent, can be accountable for the creation of the so-called "filter bubbles". This dangerous side effect of personalization turns news consumption into a one-sided propaganda by serving stories that only reaffirm one's biases. This issue came to the forefront with a vengeance after the recent unexpected election of the U.S. president Donald Trump, which was largely attributed to the dissemination of fake news over the internet [7]. According to Chaslot \& Gorbatai [39], 80\% of recommended videos on YouTube favored Trump over Clinton (regardless of the posed queries), most of which featured false stories.

How can users gain control over the aforementioned biases introduced by the RS providers? Is it users' moral right to receive recommendations that best match their interests? The freedom to choose what criteria the recommender system is optimized for may increase user satisfaction and trust through better personalization. We therefore believe that the RS's marketing bias should become adjustable by users, giving them the ability to reset the system's current optimization goals to the default, or "best match", mode. To provide evidence that a 
such a system is indeed free from biases, it can expose explanation interfaces, as discussed earlier. However, to fully address the problem of behavior manipulation, a regulatory oversight by trusted third parties is advised [107].

Online experiments

RS practitioners willing to assess the real effect of a new recommendation algorithm (or modifications of an existing one) often embark on $A / B$ testing. This is an online between-subjects experiment, where control and treatment groups are assigned the original and the modified variants of an algorithm, respectively. Digital businesses ranging from small start-ups to internet giants such as Yahoo, Twitter and Ebay, continuously run online experiments in attempt to improve their services. In the vast majority of cases, however, users are silently dragged into these experiments without their knowledge, let alone consent. Taken to an extreme, the exposure to a previously untested algorithm is somewhat akin to medical trials, where the consequences of applying a new method may lead to unpredictable side effects and potential harm.

It is worth mentioning two (in)famous examples of online experiments, which have clearly stepped over the ethical line. In 2012, Facebook studied the phenomenon of emotional contagion by intentionally manipulating the moods of more than 600,000 users through their news feeds. Two years later, dating service OKCupid studied the emotional effect of recommending bad partner matches to its users while presenting them as "exceptionally good”. Both experiments failed to acquire explicit permission from participants, while toying with their emotions and private lives. This gave rise to a public uproar and debates about the ethics of A/B testing. Referring to the aforementioned cases, Grimmelmann [73] reminds that running experiments on people is regulated by laws and as a minimum, requires the provision of informed consent and approval of an In- 
stitutional Review Board (IRB). Researchers embarking on A/B testing need to establish whether the data protection laws fall under the jurisdiction of countries where researchers or participants reside, where the server or the IRB are located, where the data is analysed, or some combination of these [172].

There are several ways of dealing with informed consent in online experiments. In the "opt-in" scenario, user consent is acquired explicitly via a prior notice. Given the ubiquity of online experiments (we note the ubiquity of RS as well), implementing prior consent in practice does not appear particularly realistic. Furthermore, prior consent introduces an unavoidable bias that may potentially influence the results and make the sampling less than random [159, 172]. Another possibility is the "implied consent" acquired through ToS, with an added option to opt-out from experiments at any time. Implied consent may be sufficient for experiments with low risk [63]. Since many companies perform A/B testing with no consent at all, the "notify after" approach can be taken to retroactively inform users about the performed study, providing them with the results, contact information of the involved researchers, and offering the option to delete their data [159]. Felten [63] warns that this alternative is only applicable to situations with minimal risk. Thus, the perceived moral impact of the experiment should be a deciding factor for obtaining informed consent. It is important to remember that when the risk of moral hazard is high, the test is unethical even with consent from participants [63].

When a recommender system is A/B tested for potential gains from applying an experimental algorithm, the two variants of algorithms (i.e. original and experimental) may differ significantly in their output. For example, hotel booking operators Expedia and Hotels.com are known for conducting A/B testing experiments that steer one of the user groups towards more expensive hotels [79]. This raises the question of fairness: is it users' moral right to demand the interaction with the trusted ("default") system instead of the one whose behav- 
ior is far less obvious? Again, we suggest that the ability to reset the recommender system to its default algorithm is provided as an option to $\mathrm{RS}$ users. Considering that $\mathrm{A} / \mathrm{B}$ testing is undoubtedly an indispensable evaluation tool in a RS developer's arsenal, it is important to ensure that it is practiced in an ethical manner.

\section{Summary as a framework}

In this section we summarize the findings of the study in the form of a user-centric ethical recommendation framework. The framework maps various RS development stages to potential ethical concerns and the recommended countermeasures. As a practical contribution, we propose an "ethical toolbox" comprised of user-adjustable controls corresponding to each development stage. These controls enable users to adjust the recommender system to their individual moral standards. Table 16 outlines the articulated framework, which also details the meaning of each component of a toolbox. 


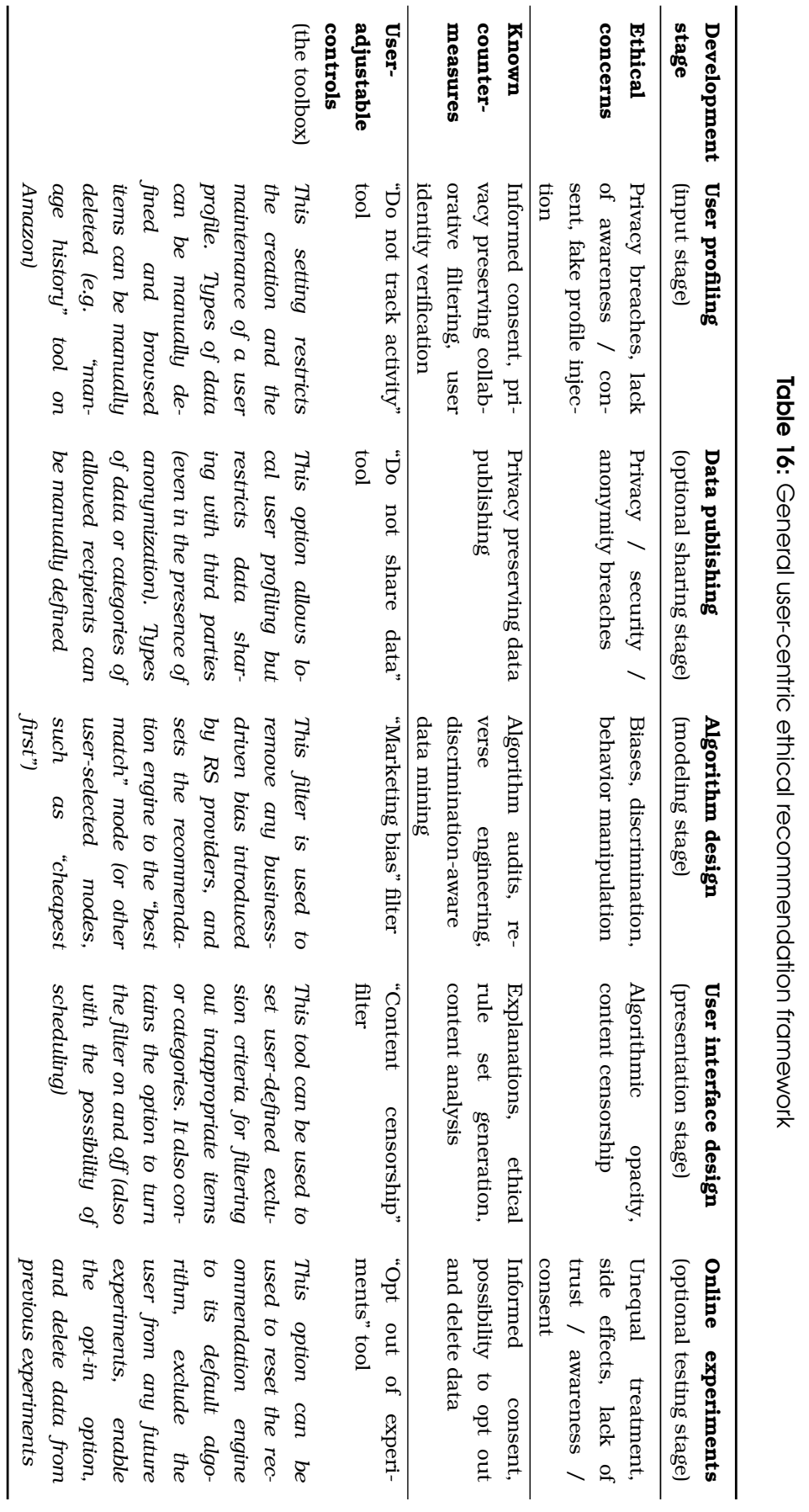


The provided transparency and control over recommendations are known to increase users' trust in the system [84, 105]. At the same time, empirical studies show that users tend to avoid the hassle of adjusting settings once they get the ability to do so [105]. However, we argue that the existence of the proposed controls is what makes the system ethics-aware by design and thus helps to retain prudent RS users who would stop using the system otherwise. One way of reducing the burden of adjusting settings is to implement adaptive defaults. For example, the "do not track activity" control can be pre-set according to the user's expressed willingness to accept cookies, or according to the estimated sensitivity of information involved in user profiling. Likewise, the content censorship filter may automatically enable itself during periods when children are expected to be at home. Certain controls, such as "do not track activity" and "do not share data" can be combined to simplify user interface. In general, the usability of the provided controls may depend on many factors, such as their layout, frequency of using the system, sensitivity of data, and so on. As a vital first step, however, it is necessary to establish the general stance of users towards the ethics of recommender systems and whether the proposed toolbox would stand as a viable solution. This is done in the next section.

\section{Feasibility Study}

We conduct an online survey ${ }^{22}$ to find out people's opinions and their preferred course of action regarding five ethical aspects of recommender systems that the proposed toolbox aims to address, namely: user profiling, data sharing, marketing bias, content censorship, and online experiments (corresponding to the five stages of the framework in Table 16). The survey was disseminated to Facebook groups of numerous European universities, yielding 224 responses from students and academic

${ }^{22}$ available at http://recommendations . typeform. com/to/kgKNQo 
staff. 10 responses from people who claimed to be totally unfamiliar with recommender systems were filtered out, leaving us with 214 valid opinions. Instead of directly asking whether participants would be willing to have a manual configuration tool for a particular ethical issue, we offered them a choice between several alternative options. The results of the survey are summarized in Tables 17-21, where answers are listed in descending order of the number of votes received. To mitigate ambiguities, each question was preceded with a short explanation of a particular ethical aspect (omitted from Tables 17-21 for brevity). We note that some complementary questions allow multiple answers (hence percentages do not always sum up to 100). 
Table 17: Issue 1/5: User profiling

\section{Q1.1. Which of the following factors, if any, would make \# (\% cases) you give up on personalization in favor of preserving pri- vacy? (multiple answers allowed)}

A) Ability to infer sensitive information from my browsing $139(65.0 \%)$ behavior, such as my sexual status, health condition, or religious beliefs.

B) Revealing my browsing behavior to other users of the same account (for example, other family members using the same website)

C) Using my profile for targeted advertising campaigns / promotional emails

D) None of the above. I want to receive personalized recommendations and I am not concerned about privacy

E) Other

$11(5.1 \%)$

F) I

F) I don't care either about recommendations or privacy

$6(2.8 \%)$ when I browse the web

\begin{tabular}{ll}
\hline Q1.2. How should user profiling be handled? & \# (\% cases) \\
\hline $\begin{array}{l}\text { A) Recommendation engines should provide user-adjustable } \\
\text { controls for setting user profiling on/off manually }\end{array}$ & $169(79.0 \%)$ \\
$\begin{array}{l}\text { B) Agreeing to the Terms of Service and allowing/disallowing } \\
\text { cookies on websites makes me feel safe about my user pro- }\end{array}$ & $38(17.8 \%)$ \\
file & \\
$\begin{array}{l}\text { C) I don't care about user profiling, and I don't want to be } \\
\text { informed or asked about it }\end{array}$ & $7(3.3 \%)$ \\
D) Other & $0(0.0 \%)$ \\
\hline
\end{tabular}


Table 18: Issue 2/5: Data sharing

\begin{abstract}
Q2.1. Which of the following probable implications, if \# (\% cases) any, would make you refrain from sharing your anonymous profile, even if it would contribute to better recommendations in the future? (multiple answers allowed)
\end{abstract}

A) Hacker attacks on anonymized public data which may 123 (57.5\%) result in re-establishing my identity

B) The possibility of using my data for purposes other than 117 (54.7\%) research

C) The possibility of finding out additional information about $116(54.2 \%)$ me by matching records in different databases

D) My willingness to share data depends on how much I 96 (44.9\%) trust the particular website that gives recommendations E) None of the above. I am not afraid of publishing my $10(4.7 \%)$ anonymized profile online as long as it benefits the service I get
F) Other
$1(0.5 \%)$

Q2.2. What is your stance towards sharing your data \# (\% cases) with third parties for research purposes?

A) I want to be able to control data sharing myself in the 104 (48.6\%) settings of a recommendation engine

B) I am willing to share my data with third parties, as long as it stays anonymous. This should be inscribed in the ToS

C) I don't want my data to be shared with third parties under $45(21.0 \%)$ any circumstances, even if it has been anonymized

D) I don't have an opinion

$0(0.0 \%)$

E) Other

$0(0.0 \%)$ 
Table 19: Issue 3/5: Online experiments

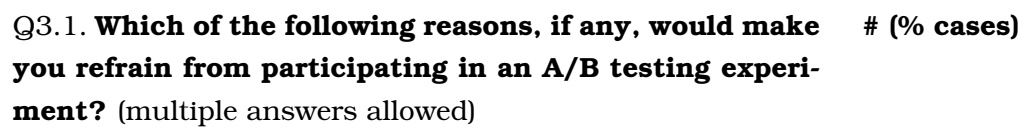

A) Lack of consent: the fact that I am not notified or asked

$103(48.1 \%)$ for permission to participate in the experiment

B) None of the above. I don't mind testing the new algorithm, 94 (43.9\%) which might work even better than the old one

C) System instability: I cannot be sure that I will get the $30(14.0 \%)$ same quality of recommendation the next time I visit the website

D) Lack of trust: I wouldn't trust an advice coming from an 25 (11.7\%) experimental, previously untested system

E) Unfairness: it is not fair to treat the users of the same $16(7.5 \%)$ system unequally

F) Other $\quad 1(0.5 \%)$

Q3.2. What would be the preferred method of getting \# (\% cases) your consent for participating in an $\mathrm{A} / \mathrm{B}$ testing experiment?

A) Contact me before the experiment and ask for my permis- 84 (39.3\%) sion to participate

B) Don't contact me at all, but provide an option in the recommendation engine for controlling whether I want to interact with the existing system or participate in testing of a new recommendation method

C) Inform me after the experiment, but allow me to delete any data I generated during the experiment, if I want to D) Don't contact me at all, and let the system do what it finds appropriate

E) Other

$1(0.5 \%)$ 
Table 20: Issue 4/5: Marketing bias

\begin{tabular}{ll}
\hline $\begin{array}{l}\text { Q4.1. What do you think about the marketing bias of a } \\
\text { recommendation system? }\end{array}$ & \# cases) \\
\hline A) I don't expect salespeople of physical stores to be unbi- & $90(42.1 \%)$ \\
ased in their recommendations. Online recommendations & \\
are no different. If it's important for the business, I don't & \\
mind & \\
B) This is clearly unethical because I expect recommenda- & $81(37.9 \%)$ \\
tions to be unbiased and tailored to my preferences at all & \\
times & \\
C) I don't mind the marketing bias because I may find such & $17(7.9 \%)$ \\
"biased" recommendations even more interesting & $16(7.5 \%)$ \\
D) I don't have an opinion & $10(4.7 \%)$ \\
E) Other & $\#(\%$ cases) \\
\hline Q4.2. How should the marketing bias be handled? & $80(37.4 \%)$ \\
\hline $\begin{array}{l}\text { A) Removing or allowing a marketing bias should become a } \\
\text { user-adjustable option of a recommendation engine }\end{array}$ & \\
B) Companies have the right to adjust their recommenda- & $60(28.0 \%)$ \\
tions as they please. Users should not intervene & \\
C) Recommendation engines should never be allowed to rec- & $49(22.9 \%)$ \\
ommend anything that doesn't serve user needs & $19(8.9 \%)$ \\
D) I don't have an opinion & $6(2.8 \%)$ \\
E) Other &
\end{tabular}

Table 21: Issue 5/5: Content censorship

\section{Q5.1. How should content censorship be handled in rec- \# (\% cases)} ommendation systems?

\begin{tabular}{|c|c|}
\hline $\begin{array}{l}\text { A) Recommendation engines should have a user-adjustable } \\
\text { censorship filter, where I can set my exclusion criteria and } \\
\text { turn the filter on/off at my own will }\end{array}$ & $104(48.6 \%)$ \\
\hline $\begin{array}{l}\text { B) There should be no censorship. I want to be able to exam- } \\
\text { ine all relevant options and judge myself what is appropriate }\end{array}$ & $58(27.1 \%)$ \\
\hline $\begin{array}{l}\text { C) Recommendation engines must filter out inappropriate } \\
\text { items automatically, based on my profile data or something } \\
\text { else }\end{array}$ & $31(14.5 \%)$ \\
\hline $\begin{array}{l}\text { D) I don't care if there is any content censorship or not in } \\
\text { recommendations }\end{array}$ & $19(8.9 \%)$ \\
\hline E) Other & $2(0.9 \%)$ \\
\hline
\end{tabular}


The examination of survey results immediately reveals participants' strong preference for taking morally sensitive issues under their control. In 4 out 5 cases, the majority voted for having a user-adjustable setting within a recommendation engine for controlling a particular ethical issue. In the following paragraphs we analyze the responses (including the free-form ones where these were deemed relevant and insightful).

Online experiments (Table 19) was the only case where a user-adjustable option came second (however with only 5.2\% difference from the top choice). Considering that the lack of consent was identified as the biggest ethical barrier to participating in A/B testing (Q3.1), the indicated preference towards the opt-in scenario (B3.2) appears well justified, as it is linked with maximal risks. As one of the participants noted, requests from users to delete their data implicitly signals that they should best be excluded from future experiments.

When it comes to user profiling (Table 17), we observe a strong trend of preferring privacy over personalization. Users' major concern is the disclosure of their browsing behavior and the risks associated with it (Q1.1). In fact, 5 respondents stated explicitly that they would not trade their privacy for personalization for whichever reason, whereas one participant stated that $\mathrm{s} /$ he would only accept personalization on a group level, while staying anonymous within the group. Our results once again confirm the failure of ToS to satisfy users' privacy needs (Q1.2). Instead, the vast majority of users (79\%) want to have the ability to control user profiling manually.

Exactly the same attitude was expressed by users towards sharing their data with third parties (Q2.2) due to various privacy concerns (Q2.1). Notably, almost half of users would adjust their data sharing strategy depending on how much they trust the particular website.

The marketing bias of RS (Table 20) brought up the most controversy as it can be seen in Q4.1, where two opposite views divided the overwhelming majority of users into two camps. 
Interestingly, the largest camp is ready to accept biased recommendations, with $7.9 \%$ of users feeling especially optimistic about them. The challenge of this particular issue can also be judged from the considerably large proportions of hesitant respondents who refrained from answering Q4.1 and Q4.2. Still, both camps prefer to have a manual control over the marketing bias in the settings of a recommender system. This issue also yielded a larger number of free-form responses, most of which starkly manifested users' negative stance towards recommendations because of the awareness of the marketing bias. One user also expressed their concern about getting falsely advertised recommendations (e.g. abusing explanations of type "other users also bought", etc.), which were mentioned in Section Algorithmic opacity, biases, and behavior manipulation.

As far as the censorship is concerned (Table 21), the preference for being able to filter the recommendation output manually clearly dominated other options in Q5.1. On the other hand, the preference for having no censorship considerably outweighed the preference for automatic content filtering. Thus, having the former option as a "smart default" is well justified. In any case, censorship appears important to the overwhelming majority of RS users (91.1\%).

\section{Conclusion}

We conclude that multiple moral dilemmas of varied severity emerge on every stage of RS development, while their solutions are not always evident or effective. In particular, there are many trade-offs to be resolved, such as user privacy vs. personalization, data anonymization vs. data utility, informed consent vs. experimentation bias, and algorithmic transparency vs. trade secrets. Balancing between consumer and corporate ethics in recommender systems is a challenging task. A careful assessment of moral risks is crucial for deciding on the strategies of data anonymization or informed consent acquisition for per- 
forming A/B testing or user profiling. Yet, the question of precisely who and how should perform such risk analysis is still a subject of debates. We have found evidence that many big players on the RS market (Facebook, Amazon, Netflix, etc.) have faced loud ethics-related backlashes. Thus, it is important to ensure that the RS architecture is not only legally and algorithmically justified, but also ethically sound.

The articulated ethical recommendation framework suggests new paradigm of ethics-awareness by design with an addition of a contextual layer that utilizes existing technologies where possible and complements them with user-adjustable controls. This idea was embraced by the vast majority of our survey participants, and future work should further test its viability in a fully implemented RS prototype. 


\section{REFERENCES}

[1] Amazon: number of active customer accounts worldwide 1997-2015. https://www.statista.com/statistics/ 237810/number-of-active-amazon-customer-accountsworldwide/, 2017. Accessed: 2017-10-18.

[2] G. Adomavicius and Y. Kwon. Multi-criteria recommender systems. In F. Ricci, L. Rokach, and B. Shapira, editors, Recommender Systems Handbook, pages 847-880. Springer US, 2015.

[3] D. Agarwal, B.-C. Chen, P. Elango, and R. Ramakrishnan. Content recommendation on web portals. Commun. ACM, 56(6):92101, June 2013.

[4] R. Agrawal, T. Imielinski, and A. Swami. Mining association rules between sets of items in large databases. In Proc. of the 1993 ACM SIGMOD International Conference on Management of Data, pages 207-216, 1993.

[5] F. Aiolli. Efficient top-n recommendation for very large scale binary rated datasets. In Proc. of the 7th ACM Conference on Recommender Systems, pages 273-280, 2013.

[6] Z. Ali, S. Khusro, and I. Ullah. A hybrid book recommender system based on table of contents (toc) and association rule mining. In Proc. of the 10th International Conference on Informatics and Systems, INFOS '16, pages 68-74. ACM, 2016.

[7] H. Allcott and M. Gentzkow. Social media and fake news in the 2016 election. NBER Working Paper No. 23089, 2017.

[8] X. Amatriain and J. Basilico. Netflix recommendations: Beyond the 5 stars. http://techblog.netflix.com/2012/ 04 /netflix-recommendations-beyond-5-stars.html, Apr. 2012. Accessed: 2015-03-12.

[9] X. Amatriain, A. Jaimes, N. Oliver, and J. M. Pujol. Data mining methods for recommender systems. In F. Ricci, L. Rokach, B. Shapira, and P. B. Kantor, editors, Recommender Systems Handbook, pages 39-71. Springer US, 2011.

[10] E. Andrews. Recommender systems for online dating. Master's thesis, University of Helsinki, 2015. 
[11] J.-Y. Audibert and S. Bubeck. Regret bounds and minimax policies under partial monitoring. J. Mach. Learn. Res., 11:27852836, Dec. 2010.

[12] P. Auer, N. Cesa-Bianchi, and P. Fischer. Finite-time analysis of the multiarmed bandit problem. Mach. Learn., 47(2-3):235-256, May 2002.

[13] F. Bakalov, M.-J. Meurs, B. König-Ries, B. Sateli, R. Witte, G. Butler, and A. Tsang. An approach to controlling user models and personalization effects in recommender systems. In Proc. of the 2013 International Conference on Intelligent User Interfaces, pages 49-56, 2013.

[14] R. Bambini, P. Cremonesi, and R. Turrin. A recommender system for an iptv service provider: a real large-scale production environment. In F. Ricci, L. Rokach, B. Shapira, and P. B. Kantor, editors, Recommender Systems Handbook, pages 299-331. Springer US, 2011.

[15] A. Bar, L. Rokach, G. Shani, B. Shapira, and A. Schclar. Improving simple collaborative filtering models using ensemble methods. In Z.-H. Zhou, F. Roli, and J. Kittler, editors, Multiple Classifier Systems: 11th International Workshop, MCS 2013, Nanjing, China, May 15-17, 2013. Proceedings, pages 1-12. Springer Berlin Heidelberg, 2013.

[16] V. A. Barger and L. Labrecque. An integrated marketing communications perspective on social media metrics. International Journal of Integrated Marketing Communications, May 2013.

[17] O. Barkan and N. Koenigstein. Item2vec: Neural item embedding for collaborative filtering. In IEEE 26th International Workshop on Machine Learning for Signal Processing (MLSP), pages 1-6, 2016.

[18] S. Barocas and H. Nissenbaum. Big data end run around anonymity and consent. In J. Lane, V. Stodden, S. Bender, and H. Nissenbaum, editors, Privacy, Big Data, and the Public Good. Frameworks for Engagement, chapter 2, pages 44-75. Cambridge University Press, 2014.

[19] J. Beel, S. Langer, M. Genzmehr, B. Gipp, C. Breitinger, and A. Nürnberger. Research paper recommender system evalua- 
tion: A quantitative literature survey. In Proc. of the International Workshop on Reproducibility and Replication in Recommender Systems Evaluation, RepSys '13, 2013.

[20] D. Ben-Shimon, A. Tsikinovsky, M. Friedmann, B. Shapira, L. Rokach, and J. Hoerle. Recsys challenge 2015 and the yoochoose dataset. In Proc. of the 9th ACM Conference on Recommender Systems, RecSys '15, pages 357-358. ACM, 2015.

[21] S. Berkovsky, N. Borisov, Y. Eytani, T. Kuflik, and F. Ricci. Examining users' attitude towards privacy preserving collaborative filtering. In Proc. of DM. UM, pages 16-22, 2007.

[22] L. Bernardi, J. Kamps, J. Kiseleva, and M. J. I. Müller. The continuous cold start problem in e-commerce recommender systems. CoRR, abs/1508.01177, June 2015.

[23] M. Blondel, A. Fujino, and N. Ueda. Convex factorization machines. In A. Appice, P. P. Rodrigues, V. Santos Costa, J. Gama, A. Jorge, and C. Soares, editors, Machine Learning and Knowledge Discovery in Databases: European Conference, ECML PKDD 2015, Porto, Portugal, September 7-11, 2015, Proceedings, Part II, pages 19-35. Springer International Publishing, 2015.

[24] A. V. Bodapati. Recommendation systems with purchase data. Journal of Marketing Research, 45(1):77-93, 2008.

[25] D. Bouneffouf and R. Féraud. Multi-armed bandit problem with known trend. Neurocomput., 205(C):16-21, Sept. 2016.

[26] D. Bouneffouf, R. Laroche, T. Urvoy, R. Feraud, and R. Allesiardo. Contextual bandit for active learning: Active thompson sampling. In C. K. Loo, K. S. Yap, K. W. Wong, A. Teoh, and K. Huang, editors, Neural Information Processing: 21st International Conference, ICONIP 2014, Kuching, Malaysia, November 3-6, 2014. Proceedings, Part I, pages 405-412. Springer International Publishing, 2014.

[27] A. P. Bradley. The use of the area under the roc curve in the evaluation of machine learning algorithms. Pattern Recogn., 30(7):1145-1159, July 1997.

[28] L. Breiman. Bagging predictors. Machine Learning, 24(2):123140, Aug 1996. 
[29] B. Brodén, M. Hammar, B. J. Nilsson, and D. Paraschakis. Bandit algorithms for e-commerce recommender systems: Extended abstract. In Proc. of the 11th ACM Conference on Recommender Systems, RecSys '17, pages 349-349. ACM, 2017.

[30] B. Brodén, M. Hammar, B. J. Nilsson, and D. Paraschakis. An ensemble recommender system for e-commerce. In 26th Benelux Conference on Machine Learning (Benelearn), 2017.

[31] B. Brodén, M. Hammar, B. J. Nilsson, and D. Paraschakis. Ensemble recommendations via Thomson Sampling: an experimental study within e-commerce. In Proc. of the 23rd ACM Conference on Intelligent User Interfaces (IUI). ACM, in press.

[32] R. Burke, M. P. O’Mahony, and N. J. Hurley. Robust collaborative recommendation. In F. Ricci, L. Rokach, and B. Shapira, editors, Recommender Systems Handbook, pages 961-995. Springer US, 2015.

[33] R. Burke and M. Ramezani. Matching recommendation technologies and domains. In F. Ricci, L. Rokach, B. Shapira, and P. B. Kantor, editors, Recommender Systems Handbook, pages 367-386. Springer US, 2011.

[34] J. Burrell. How the machine 'thinks': Understanding opacity in machine learning algorithms. Big Data \& Society, 3(1), 2016.

[35] G. Burtini, J. Loeppky, and R. Lawrence. Improving online marketing experiments with drifting multi-armed bandits. In Proc. of the 17th International Conference on Enterprise Information Systems - Volume 1, ICEIS 2015, pages 630-636. SCITEPRESS Science and Technology Publications, Lda, 2015.

[36] S. Caron and S. Bhagat. Mixing bandits: A recipe for improved cold-start recommendations in a social network. In Proc. of the 7th Workshop on Social Network Mining and Analysis, SNAKDD '13, pages 11:1-11:9. ACM, 2013.

[37] O. Chapelle and L. Li. An empirical evaluation of thompson sampling. In J. Shawe-Taylor, R. S. Zemel, P. L. Bartlett, F. Pereira, and K. Q. Weinberger, editors, Advances in Neural Information Processing Systems 24, pages 2249-2257. Curran Associates, Inc., 2011. 
[38] O. Chapelle, D. Metlzer, Y. Zhang, and P. Grinspan. Expected reciprocal rank for graded relevance. In Proc. of the 18th ACM Conference on Information and Knowledge Management, CIKM '09, pages 621-630. ACM, 2009.

[39] G. Chaslot and A. Gorbatai. Does youtubes a.i. favor trump and fake news? Online: https://medium.com/thegraph/youtubes-ai-is-neutral-towards-clicks-butis-biased-towards-people-and-ideas-3a2f643dea9a\# .gce3bifs2, 2016. Accessed: 2017-02-05.

[40] L. Cohen, L. Manion, and K. Morrison. Research methods in education. New York: Routledge/Taylor \& Francis Group, 6th edition, 2007.

[41] J. Cook. Ethics of data mining. http://scholarworks.rit. edu/article/441, 2005. Accessed: 2017-11-19.

[42] N. Craswell. Mean reciprocal rank. In L. Liu and M. T. Özsu, editors, Encyclopedia of Database Systems, pages 1703-1703. Springer US, 2009.

[43] N. Craswell, O. Zoeter, M. Taylor, and B. Ramsey. An experimental comparison of click position-bias models. In Proc. of the 2008 International Conference on Web Search and Data Mining, WSDM '08, pages 87-94. ACM, 2008.

[44] P. Cremonesi, F. Garzotto, and R. Turrin. Investigating the persuasion potential of recommender systems from a quality perspective: An empirical study. ACM Trans. Interact. Intell. Syst., 2(2):11:1-11:41, 2012.

[45] P. Cremonesi, Y. Koren, and R. Turrin. Performance of recommender algorithms on top-n recommendation tasks. In Proc. of the Fourth ACM Conference on Recommender Systems, RecSys '10, pages 39-46. ACM, 2010.

[46] P. Cremonesi, R. Turrin, and F. Airoldi. Hybrid algorithms for recommending new items. In Proc. of the 2Nd International Workshop on Information Heterogeneity and Fusion in Recommender Systems, HetRec '11, pages 33-40. ACM, 2011.

[47] E. Davoodi, K. Kianmehr, and M. Afsharchi. A semantic social network-based expert recommender system. Applied Intelligence, 39(1):1-13, 2013. 
[48] D. DeCoste. Collaborative prediction using ensembles of maximum margin matrix factorizations. In Proc. of the 23rd International Conference on Machine Learning, ICML '06, pages 249256. ACM, 2006.

[49] M. Denscombe. The good research guide: for small-scale social research projects (4th edition). Open University Press, 2010.

[50] M. Deshpande and G. Karypis. Item-based top-n recommendation algorithms. ACM Trans. Inf. Syst., 22(1):143-177, Jan. 2004.

[51] T. Di Noia and V. C. Ostuni. Recommender systems and linked open data. In W. Faber and A. Paschke, editors, Reasoning Web. Web Logic Rules: 11th International Summer School 2015, Berlin, Germany, July 31- August 4, 2015, Tutorial Lectures., pages 88113. Springer International Publishing, 2015.

[52] N. Diakopoulos. Algorithmic accountability reporting: On the investigation of black boxes. Tow Center for Digital Journalism A Tow/Knight Brief, 2014.

[53] N. Diakopoulos, S. Friedler, M. Arenas, S. Barocas, M. Hay, B. Howe, H. Jagadish, K. Unsworth, A. Sahuguet, S. Venkatasubramanian, C. Wilson, C. Yu, and B. Zevenbergen. Principles for accountable algorithms and a social impact statement for algorithms. Online: https://www.fatml.org/resources/ principles-for-accountable-algorithms/. Accessed: 2017-10-25.

[54] T. G. Dietterich. Ensemble methods in machine learning. In Proc. of the First International Workshop on Multiple Classifier Systems, MCS '00, pages 1-15. Springer-Verlag, 2000.

[55] T. Dunning. Accurate methods for the statistics of surprise and coincidence. Comput. Linguist., 19(1):61-74, 1993.

[56] T. Dunning. Surprise and coincidence - musings from the long tail. Online: http://tdunning.blogspot.se/2008/ 03/surprise-and-coincidence.html, mar 2008. Accessed: 2015-03-09.

[57] C. Dwork and A. Roth. The algorithmic foundations of differential privacy. Foundations and Trends in Theoretical Computer Science, 9(3-4):211-407, 2014. 
[58] Y. El Madani El Alami, E. H. Nfaoui, and B. O. E. Toward an effective hybrid collaborative filtering: A new approach based on matrix factorization and heuristic-based neighborhood. In Intelligent Systems and Computer Vision (ISCV), pages 1-8, 2015.

[59] . Executive Office of the President. Big data: Seizing opportunities, preserving values. Online: www.whitehouse.gov/sites/ default/files/docs/big_data_privacy_report_may_1_ 2014 .pdf/, 2014. Accessed: 2016-04-09.

[60] C. Fagan. Algorithmic accountability. Technical report, World Wide Web Foundation, July 2017.

[61] K. Faisal, T. Calders, and M. Pechenizkiy. Techniques for discrimination-free predictive models. In B. Custers, T. Calders, B. Schermer, and T. Zarsky, editors, Discrimination and Privacy in the Information Society, chapter 12, pages 223-239. Springer Berlin Heidelberg, 2013.

[62] C. Z. Felício, K. V. Paixão, C. A. Barcelos, and P. Preux. A multiarmed bandit model selection for cold-start user recommendation. In Proc. of the 25th Conference on User Modeling, Adaptation and Personalization, UMAP '17, pages 32-40. ACM, 2017.

[63] E. Felten. On the ethics of $a / b$ testing. Online: freedom-to-tinker.com/blog/felten/on-the-ethicsof-ab-testing/, 2014. Accessed: 2016-05-15.

[64] L. Floridi and J. W. Sanders. On the morality of artificial agents. Minds Mach., 14(3):349-379, Aug. 2004.

[65] A. Friedman, B. Knijnenburg, K. Vanhecke, L. Martens, and S. Berkovsky. Privacy aspects of recommender systems. In F. Ricci, L. Rokach, and B. Shapira, editors, Recommender Systems Handbook (2nd edition), chapter 19, pages 649-688. Springer Science+Business Media New York, 2015.

[66] Z. Gantner, S. Rendle, C. Freudenthaler, and L. SchmidtThieme. Mymedialite: A free recommender system library. In Proc. of the 5th ACM Conference on Recommender Systems, RecSys $2011,2011$.

[67] A. Garivier and O. Cappé. The KL-UCB algorithm for bounded stochastic bandits and beyond. In The 24th Annual Conference on Learning Theory, pages 359-376, 2011. 
[68] A. Garivier and E. Moulines. On upper-confidence bound policies for switching bandit problems. In Proc. of the 22Nd International Conference on Algorithmic Learning Theory, ALT'11, pages 174-188. Springer-Verlag, 2011.

[69] B. Gert. Common morality: Deciding what to do. Oxford University Press, 2004.

[70] S. Geuens. Factorization machines for hybrid recommendation systems based on behavioral, product, and customer data. In Proc. of the 9th ACM Conference on Recommender Systems, RecSys '15, pages 379-382. ACM, 2015.

[71] A. Gopalan, S. Mannor, and Y. Mansour. Thompson sampling for complex online problems. In Proc. of the 31st International Conference on International Conference on Machine Learning Volume 32, ICML'14, pages I-100-I-108. JMLR.org, 2014.

[72] P. Grey. How many products does amazon sell? https: //export-x.com/2014/08/14/many-products-amazonsel1-2/, Aug. 2014. Accessed: 2017-10-18.

[73] J. Grimmelmann. Illegal, immoral, and mood-altering. how facebook and okcupid broke the law when they experimented on users. Online: medium.com/@JamesGrimmelmann/illegalunethical-and-mood-altering-8b93af772688, 2014. Accessed: 2016-05-14.

[74] F. Guillou. On recommendation systems in a sequential context. PhD thesis, Université Charles de Gaulle - Lille III, Dec. 2016.

[75] A. Gunawardana and G. Shani. Evaluating recommender systems. In F. Ricci, L. Rokach, and B. Shapira, editors, Recommender Systems Handbook, pages 265-308. Springer US, 2015.

[76] J. Hagen-Zanker and R. Mallett. How to do a rigorous, evidencefocused literature review in international development. Technical report, Overseas Development Institute, 2013.

[77] M. Hammar, R. Karlsson, and B. J. Nilsson. Using maximum coverage to optimize recommendation systems in e-commerce. In Proc. of the 7th ACM Conference on Recommender Systems, RecSys '13, pages 265-272. ACM, 2013.

[78] J. Han, J. Pei, Y. Yin, and R. Mao. Mining frequent patterns without candidate generation: A frequent-pattern tree ap- 
proach. Data Mining and Knowledge Discovery, 8(1):53-87, Jan 2004.

[79] A. Hannak, G. Soeller, D. Lazer, A. Mislove, and C. Wilson. Measuring price discrimination and steering on e-commerce web sites. In Proc. of the 2014 Conference on Internet Measurement Conference, IMC '14, pages 305-318, 2014.

[80] N. Hariri, B. Mobasher, and R. Burke. Adapting to user preference changes in interactive recommendation. In Proc. of the 24th International Conference on Artificial Intelligence, IJCAI'15, pages 4268-4274. AAAI Press, 2015.

[81] C. Hartland, S. Gelly, N. Baskiotis, O. Teytaud, and M. Sebag. Multi-armed bandit, dynamic environments and meta-bandits. In Online Trading of Exploration and Exploitation, NIPS 2006 Workshop, 2006.

[82] B. Heitmann and C. Hayes. Using linked data to build open, collaborative recommender systems. In AAAI Spring Symposium: Linked Data Meets Artificial Intelligence, pages 76-81, 2010.

[83] P. J. Henrichsen. Ethical intelligence in social recommender systems. In Proceeding of the 15th International Conference on Intelligent User Interfaces, 2010.

[84] J. L. Herlocker, J. A. Konstan, and J. Riedl. Explaining collaborative filtering recommendations. In Proc. of the $2000 \mathrm{ACM}$ Conference on Computer Supported Cooperative Work, CSCW '00, pages 241-250. ACM, 2000.

[85] A. Hevner and S. Chatterjee. Design science research in information systems. In Design Research in Information Systems: Theory and Practice, pages 9-22. Springer US, 2010.

[86] A. R. Hevner, S. T. March, J. Park, and S. Ram. Design science in information systems research. MIS Q., 28(1):75-105, 2004.

[87] B. Hidasi, A. Karatzoglou, L. Baltrunas, and D. Tikk. Sessionbased recommendations with recurrent neural networks. In CoRR, abs/1511.06939, 2015.

[88] D. Howard and I. Muntean. A minimalist model of the artificial autonomous moral agent (aama). In SSS-16 Symposium Technical Reports. Association for the Advancement of Artificial Intelligence. AAAI, 2016. 
[89] C.-C. Hsieh, J. Neufeld, T. King, and J. Cho. Efficient approximate thompson sampling for search query recommendation. In Proc. of the 30th Annual ACM Symposium on Applied Computing, SAC '15, pages 740-746. ACM, 2015.

[90] Y. Hu, Y. Koren, and C. Volinsky. Collaborative filtering for implicit feedback datasets. In Proc. of the 2008 Eighth IEEE International Conference on Data Mining, ICDM '08, pages 263-272, 2008.

[91] K.-H. Huang and H.-T. Lin. Linear upper confidence bound algorithm forźcontextual bandit problem with piled rewards. In Proceedings, Part II, of the 20th Pacific-Asia Conference on Advances in Knowledge Discovery and Data Mining - Volume 9652, PAKDD 2016, pages 143-155. Springer-Verlag New York, Inc., 2016.

[92] Z. Huang, D. Zeng, and H. Chen. A comparative study of recommendation algorithms for e-commerce applications. IEEE Intelligent Systems, 2006.

[93] J. S. Hunter and T. H. Naylor. Experimental designs for computer simulation experiments. Management Science, 16(7):422$434,1970$.

[94] M. Jahrer, A. Töscher, and R. Legenstein. Combining predictions for accurate recommender systems. In Proc. of the 16th ACM SIGKDD International Conference on Knowledge Discovery and Data Mining, KDD '10, pages 693-702. ACM, 2010.

[95] D. Jannach and M. Ludewig. When recurrent neural networks meet the neighborhood for session-based recommendation. In Proc. of the Eleventh ACM Conference on Recommender Systems, RecSys '17, pages 306-310. ACM, 2017.

[96] T. D. Jick. Mixing qualitative and quantitative methods: Triangulation in action. Administrative Science Quarterly, 24(4):602$611,1979$.

[97] C. C. Johnson. Logistic matrix factorization for implicit feedback data. In NIPS Workshop on Distributed Matrix Computations, 2014.

[98] T. Kamishima and S. Akaho. Personalized pricing recommender system: Multi-stage epsilon-greedy approach. In Proc. of the 2nd 
International Workshop on Information Heterogeneity and Fusion in Recommender Systems, pages 57-64. ACM, 2011.

[99] V. Kanade, B. Mcmahan, and B. Brent. Sleeping experts and bandits with stochastic action availability and adversarial rewards. In In Proc. of the Twelfth International Conference on Artificial Intelligence and Statistics, number 5, pages 272-279, 2009.

[100] E. Kaufmann, N. Korda, and R. Munos. Thompson sampling: An asymptotically optimal finite-time analysis. In Proc. of the 23rd International Conference on Algorithmic Learning Theory, ALT'12, pages 199-213. Springer-Verlag, 2012.

[101] J. Kawale, H. H. Bui, B. Kveton, L. Tran-Thanh, and S. Chawla. Efficient thompson sampling for online matrix factorization recommendation. In Advances in Neural Information Processing Systems 28, pages 1297-1305. Curran Associates, Inc., 2015.

[102] C. Kim and J. Kim. A recommendation algorithm using multilevel association rules. In Proc. of the 2003 IEEE/WIC International Conference on Web Intelligence, WI '03, 2003.

[103] R. Kleinberg, A. Niculescu-Mizil, and Y. Sharma. Regret bounds for sleeping experts and bandits. Mach. Learn., 80(2-3):245-272, Sept. 2010.

[104] B. Knijnenburg. Simplifying privacy decisions: Towards interactive and adaptive solutions. In Decisions@ RecSys, pages 40-41, 2013.

[105] B. Knijnenburg. A User-Tailored Approach to Privacy Decision Support. PhD thesis, University of California, 2015.

[106] T. Kocák, M. Valko, R. Munos, and S. Agrawal. Spectral thompson sampling. In Proc. of the Twenty-Eighth AAAI Conference on Artificial Intelligence, AAAI'14, pages 1911-1917. AAAI Press, 2014.

[107] A. Koene, E. Perez, C. J. Carter, R. Statache, S. Adolphs, C. O'Malley, T. Rodden, and D. McAuley. Ethics of personalized information filtering. In Second International Conference on Internet Science, INSCI, pages 123-132, 2015.

[108] A. E. Kohlhase and M. Kohlhase. Semantic transparency in user assistance systems. In Proc. of the 27th ACM International Con- 
ference on Design of Communication, SIGDOC '09, pages 89-96. ACM, 2009.

[109] J. Komiyama, J. Honda, and H. Nakagawa. Optimal regret analysis of thompson sampling in stochastic multi-armed bandit problem with multiple plays. In Proc. of the 32Nd International Conference on International Conference on Machine Learning Volume 37, ICML'15, pages 1152-1161. JMLR.org, 2015.

[110] Y. Koren. Factorization meets the neighborhood: A multifaceted collaborative filtering model. In Proc. of the 14th ACM SIGKDD International Conference on Knowledge Discovery and Data Mining, KDD '08, pages 426-434. ACM, 2008.

[111] Y. Koren. Factor in the neighbors: Scalable and accurate collaborative filtering. ACM Trans. Knowl. Discov. Data, 4(1):1:1-1:24, Jan. 2010.

[112] Y. Koren, R. Bell, and C. Volinsky. Matrix factorization techniques for recommender systems. Computer, 42(8):30-37, Aug 2009.

[113] B. Kveton, C. Szepesvári, Z. Wen, and A. Ashkan. Cascading bandits: Learning to rank in the cascade model. In Proc. of the 32Nd International Conference on International Conference on Machine Learning - Volume 37, ICML'15, pages 767-776. JMLR.org, 2015.

[114] A. Lacerda. Multi-objective ranked bandits for recommender systems. Neurocomput., 246(C):12-24, July 2017.

[115] T. Lai and H. Robbins. Asymptotically efficient adaptive allocation rules. Adv. Appl. Math., 6(1):4-22, Mar. 1985.

[116] G. Li and Q. Chen. Exploiting explicit and implicit feedback for personalized ranking. Mathematical Problems in Engineering, pages $1-11,2016$.

[117] L. Li, W. Chu, J. Langford, and R. E. Schapire. A contextualbandit approach to personalized news article recommendation. In Proc. of the 19th International Conference on World Wide Web, WWW'10, pages 661-670. ACM, 2010.

[118] L. Li, W. Chu, J. Langford, and X. Wang. Unbiased offline evaluation of contextual-bandit-based news article recommendation 
algorithms. In Proc. of the Fourth ACM International Conference on Web Search and Data Mining, WSDM '11, pages 297-306. ACM, 2011.

[119] S. Li, A. Karatzoglou, and C. Gentile. Collaborative filtering bandits. In Proc. of the 39th International ACM SIGIR Conference on Research and Development in Information Retrieval, SIGIR '16, pages 539-548. ACM, 2016.

[120] S. Li, B. Wang, S. Zhang, and W. Chen. Contextual combinatorial cascading bandits. In Proc. of the 33rd International Conference on International Conference on Machine Learning - Volume 48, ICML'16, pages 1245-1253. JMLR.org, 2016.

[121] G. Linden, B. Smith, and J. York. Amazon.com recommendations: Item-to-item collaborative filtering. IEEE Internet Computing, 7(1):76-80, Jan. 2003.

[122] P. Lops, M. de Gemmis, and G. Semeraro. Content-based recommender systems: State of the art and trends. In F. Ricci, L. Rokach, B. Shapira, and P. B. Kantor, editors, Recommender Systems Handbook, pages 73-105. Springer US, 2011.

[123] J. Louëdec, M. Chevalier, J. Mothe, A. Garivier, and S. Gerchinovitz. A multiple-play bandit algorithm applied to recommender systems. In Proc. of the Twenty-Eighth International Florida Artificial Intelligence Research Society Conference, FLAIRS, pages 67-72, 2015.

[124] A. Machanavajjhala, D. Kifer, J. Gehrke, and M. Venkitasubramaniam. L-diversity: Privacy beyond k-anonymity. ACM Transactions on Knowledge Discovery from Data, 1(1), Mar. 2007.

[125] C. D. Manning, P. Raghavan, and H. Schutze. Evaluation in information retrieval. In Introduction to Information Retrieval, pages 151-175. Cambridge University Press, 2008.

[126] S. T. March and G. F. Smith. Design and natural science research on information technology. Decis. Support Syst., 15(4):251-266, 1995.

[127] M. Marshall. Aggregate knowledge raises $\$ 5 \mathrm{~m}$ from kleiner, on a roll. http://venturebeat.com/2006/12/10/aggregateknowledge-raises-5m-from-kleiner-on-a-roll/, Dec. 2006. Accessed: 2015-03-12. 
[128] J. Mary, R. Gaudel, and P. Preux. Bandits warm-up cold recommender systems. CoRR, abs/1407.2806, June 2014.

[129] M. Massoud and M. Abo-Rizka. A conceptual model of personalized pricing recommender system based on customer online behavior. International Journal of Computer Science \& Network Security, 12(6):129-133, 2012.

[130] A. M. McDonald and C. L. Faith. The cost of reading privacy policies. I/S: A Journal of Law and Policy for the Information Society, 4:540-565, 2008.

[131] J. Mellor and J. Shapiro. Thompson sampling in switching environments with bayesian online change point detection. In Proc. of the Sixteenth International Conference on Artificial Intelligence and Statistics, pages 442-450, 2013.

[132] J. Metcalf. Letter on proposed changes to the common rule. Online: bdes.datasociety.net/council-output/letter-onproposed-changes-to-the-common-rule/, December 2015. Accessed: 2016-04-08.

[133] V. Mnih, A. P. Badia, M. Mirza, A. Graves, T. Lillicrap, T. Harley, D. Silver, and K. Kavukcuoglu. Asynchronous methods for deep reinforcement learning. In M. F. Balcan and K. Q. Weinberger, editors, Proc. of The 33rd International Conference on Machine Learning, volume 48 of Proc. of Machine Learning Research, pages 1928-1937. PMLR, 20-22 Jun 2016.

[134] V. Mnih, K. Kavukcuoglu, D. Silver, A. A. Rusu, J. Veness, M. G. Bellemare, A. Graves, M. Riedmiller, A. K. Fidjeland, G. Ostrovski, S. Petersen, C. Beattie, A. Sadik, I. Antonoglou, H. King, D. Kumaran, D. Wierstra, S. Legg, and D. Hassabis. Human-level control through deep reinforcement learning. $\mathrm{Na}$ ture, 518(7540):529-533, Feb. 2015.

[135] B. Mobasher, H. Dai, T. Luo, and M. Nakagawa. Effective personalization based on association rule discovery from web usage data. In Proc. of the 3rd international workshop on Web information and data management, pages 9-15. ACM, 2001.

[136] J. H. Moor. Just consequentialism and computing. Ethics and Inf. Technol., 1(1):61-65, Jan. 1998. 
[137] J. H. Moor. The nature, importance, and difficulty of machine ethics. IEEE Intelligent Systems, 21(4):18-21, July 2006.

[138] C. Musto, G. Semeraro, M. de Gemmis, and P. Lops. Learning word embeddings from wikipedia for content-based recommender systems. In N. Ferro, F. Crestani, M.-F. Moens, J. Mothe, F. Silvestri, G. M. Di Nunzio, C. Hauff, and G. Silvello, editors, Advances in Information Retrieval: 38th European Conference on IR Research, ECIR 2016, Padua, Italy, March 2023, 2016. Proceedings, pages 729-734. Springer International Publishing, 2016.

[139] A. Narayanan and V. Shmatikov. Robust de-anonymization of large sparse datasets. In Proc. of the 2008 IEEE Symposium on Security and Privacy, pages 111-125. IEEE Computer Society, 2008.

[140] A. Narayanan and V. Shmatikov. De-anonymizing social networks. In 30th IEEE Symposium on Security and Privacy, pages 173-187. IEEE, 2009.

[141] T. Nedelec, E. Smirnova, and F. Vasile. Specializing joint representations for the task of product recommendation. In Proc. of the 2Nd Workshop on Deep Learning for Recommender Systems, DLRS 2017, pages 10-18. ACM, 2017.

[142] M.-Q. Nguyen. Multi-armed bandit problem and its applications in intelligent tutoring systems. Master's thesis, cole Polytechnique, 2014.

[143] H. Nissenbaum. Computing and accountability. Commun. ACM, 37(1):72-80, Jan. 1994.

[144] H. Nissenbaum. Human values and the design of computer technology. chapter Accountability in a Computerized Society, pages 41-64. Center for the Study of Language and Information, 1997.

[145] B. J. Oates. Researching information systems and computing. Sage Publications Ltd., 2006.

[146] J. Obar. Big data and the phantom public: Walter lippmann and the fallacy of data privacy self-management. Big Data \& Society, 2015. 
[147] P. Ohm. Broken promises of privacy: Responding to the surprising failure of anonymization. UCLA Law Review, 57:1701, 2009.

[148] S. Owen, R. Anil, T. Dunning, and E. Friedman. Mahout in action. Manning Publications Co., 2011.

[149] R. Pan, Y. Zhou, B. Cao, N. N. Liu, R. Lukose, M. Scholz, and Q. Yang. One-class collaborative filtering. In Proc. of the 2008 Eighth IEEE International Conference on Data Mining, ICDM '08, pages 502-511, 2008.

[150] D. Paraschakis. Recommender systems from an industrial and ethical perspective. In Proc. of the 10th ACM Conference on Recommender Systems, RecSys '16, pages 463-466. ACM, 2016.

[151] D. Paraschakis. Towards an ethical recommendation framework. In Proc. of the 11th International Conference on Research Challenges in Information Science (RCIS), pages 211 - 220. IEEE, 2017.

[152] D. Paraschakis, B. J. Nilsson, and J. Holländer. Comparative evaluation of top- $\mathrm{N}$ recommenders in e-commerce: An industrial perspective. In Proc. of the 14th International Conference on Machine Learning and Applications (ICMLA), pages 1024 - 1031. IEEE, 2015.

[153] S.-T. Park and W. Chu. Pairwise preference regression for coldstart recommendation. In Proc. of the Third ACM Conference on Recommender Systems, RecSys '09, pages 21-28. ACM, 2009.

[154] F. Pasquale. The black box society. the secret algorithms that control money and information. Harvard University Press, 2015.

[155] M. J. Pazzani and D. Billsus. Content-based recommendation systems. In P. Brusilovsky, A. Kobsa, and W. Nejdl, editors, The Adaptive Web: Methods and Strategies of Web Personalization, pages 325-341. Springer Berlin Heidelberg, 2007.

[156] A. Pinsonneault and K. L. Kraemer. Survey research methodology in management information systems: An assessment. J. Manage. Inf. Syst., 10(2):75-105, Sept. 1993.

[157] H. Polat and W. Du. Privacy-preserving collaborative filtering using randomized perturbation techniques. In Proc. of the Third 
IEEE International Conference on Data Mining, pages 625-. IEEE Computer Society, 2003.

[158] B. Pradel, S. Sean, J. Delporte, S. Guérif, C. Rouveirol, N. Usunier, F. Fogelman-Soulié, and F. Dufau-Joel. A case study in a recommender system based on purchase data. In Proc. of the 17th ACM SIGKDD International Conference on Knowledge Discovery and Data Mining, KDD '11, pages 377-385, 2011.

[159] C. Puschmann and E. Bozdag. Staking out the unclear ethical terrain of online social experiments. Internet Policy Review, 3, nov 2014.

[160] M. Quadrana. Algorithms for Sequence-Aware Recommender Systems. PhD thesis, Politecnico di Milano, 2017.

[161] M. Quadrana, A. Karatzoglou, B. Hidasi, and P. Cremonesi. Personalizing session-based recommendations with hierarchical recurrent neural networks. In Proc. of the Eleventh ACM Conference on Recommender Systems, RecSys '17, pages 130-137. ACM, 2017.

[162] F. Radlinski, R. Kleinberg, and T. Joachims. Learning diverse rankings with multi-armed bandits. In Proc. of the 25th International Conference on Machine Learning, ICML '08, pages 784791. ACM, 2008.

[163] N. Ramakrishnan, B. J. Keller, B. J. Mirza, A. Y. Grama, and G. Karypis. Privacy risks in recommender systems. IEEE Internet Computing, 5(6):54-62, 2001.

[164] S. Rendle. Factorization machines. In Proc. of the 2010 IEEE International Conference on Data Mining, ICDM '10, pages 9951000. IEEE Computer Society, 2010.

[165] S. Rendle, C. Freudenthaler, Z. Gantner, and L. SchmidtThieme. Bpr: Bayesian personalized ranking from implicit feedback. In Proc. of the Twenty-Fifth Conference on Uncertainty in Artificial Intelligence, UAI '09, 2009.

[166] J. D. M. Rennie and N. Srebro. Fast maximum margin matrix factorization for collaborative prediction. In Proc. of the 22Nd International Conference on Machine Learning, ICML '05, pages 713-719. ACM, 2005. 
[167] F. Ricci, L. Rokach, and B. Shapira. Introduction to recommender systems handbook. In F. Ricci, L. Rokach, B. Shapira, and P. B. Kantor, editors, Recommender Systems Handbook, pages 1-35. Springer US, 2011.

[168] J. Rishabh. When recommendation systems go bad. Online: cds.nyu.edu/recommendation-systems-go-bad/, 2016. Accessed: 2016-05-15.

[169] M. Rodriguez and J. Watkins. Faith in the algorithm, part 2: Computational eudaemonics. In 13th International Conference on Knowledge-Based and Intelligent Information and Engineering Systems: Part II, pages 813-820. Springer Berlin Heidelberg, 2009.

[170] T. Rohle. Desperately seeking the consumer: Personalized search engines and the commercial exploitation of user data. Online: journals.uic.edu/ojs/index.php/fm/ article/view/2008/18831, sept 2007. Accessed: 2016-04-08.

[171] J. Rongfei, J. Maozhong, and L. Chao. A new clustering method for collaborative filtering. In Proc. of the International Conference on Networking and Information Technology, pages 488-492, June 2010.

[172] M. Ross. Do research ethics need updating for the digital age? Monitor on Psychology, 45(9):64, oct 2014.

[173] A. Said, A. Bellogin, and A. de Vries. A top-n recommender system evaluation protocol inspired by deployed systems. LSRS Workshop at ACM RecSys, 2013.

[174] R. Salakhutdinov and A. Mnih. Probabilistic matrix factorization. In Proc. of the 20th International Conference on Neural Information Processing Systems, NIPS'07, pages 1257-1264. Curran Associates Inc., 2007.

[175] S. M. Sanchez. Work smarter, not harder: guidelines for designing simulation experiments. In Proc. of the Winter Simulation Conference, 2005., pages 14 pp.-, Dec 2005.

[176] C. Sandvig, K. Hamilton, K. Karahalios, and C. Langbort. Auditing algorithms: Research methods for detecting discrimination on internet platforms. In Pre-conference of the 64th annual meeting of the International Communication Association, 2014. 
[177] B. Sarwar, G. Karypis, J. Konstan, and J. Riedl. Analysis of recommendation algorithms for e-commerce. In Proc. of the $2 \mathrm{Nd}$ ACM Conference on Electronic Commerce, EC 'O0, pages 158167, 2000.

[178] B. Sarwar, G. Karypis, J. Konstan, and J. Riedl. Item-based collaborative filtering recommendation algorithms. In Proc. of the 10th International Conference on World Wide Web, WWW '01, pages 285-295. ACM, 2001.

[179] B. Sarwar, G. Karypis, J. Konstan, and J. Riedl. Incremental singular value decomposition algorithms for highly scalable recommender systems. pages 27-28, 2002.

[180] J. B. Schafer, J. A. Konstan, and J. Riedl. E-commerce recommendation applications. Data Min. Knowl. Discov., 5(1-2):115153, Jan. 2001.

[181] R. E. Schapire. A brief introduction to boosting. In Proc. of the 16th International Joint Conference on Artificial Intelligence Volume 2, IJCAI'99, pages 1401-1406. Morgan Kaufmann Publishers Inc., 1999.

[182] A. I. Schein, A. Popescul, L. H. Ungar, and D. M. Pennock. Methods and metrics for cold-start recommendations. In Proc. of the 25th Annual International ACM SIGIR Conference on Research and Development in Information Retrieval, SIGIR '02, pages 253260. ACM, 2002.

[183] B. Schwartz. The paradox of choice: why more is less. ECCO New York, 1st ed. edition, 2004.

[184] A. Slivkins. Contextual bandits with similarity information. J. Mach. Learn. Res., 15(1):2533-2568, Jan. 2014.

[185] A. Slivkins, F. Radlinski, and S. Gollapudi. Ranked bandits in metric spaces: Learning diverse rankings over large document collections. J. Mach. Learn. Res., 14(1):399-436, Feb. 2013.

[186] K. Souali, A. El Afia, and R. Faizi. An automatic ethical-based recommender system for e-commerce. In International Conference on Multimedia Computing and Systems, pages 1-4. IEEE, 2011. 
[187] H. Stormer. Improving e-commerce recommender systems by the identification of seasonal products. In Conference on Artificial Intelligence, pages 92-99, 2007.

[188] M. Streeter and D. Golovin. An online algorithm for maximizing submodular functions. In Proc. of the 21st International Conference on Neural Information Processing Systems, NIPS'08, pages 1577-1584. Curran Associates Inc., 2008.

[189] L. Sweeney. Uniqueness of simple demographics in the u.s. population. Technical report, Carnegie Mellon University, School of Computer Science. Data Privacy Laboratory, Technical Report LIDAP-WP4, 2000.

[190] L. Sweeney. K-anonymity: A model for protecting privacy. International Journal of Uncertainty, Fuzziness and Knowledge-Based Systems, 10(5):557-570, 2002.

[191] G. Takács, I. Pilászy, B. Németh, and D. Tikk. Major components of the gravity recommendation system. SIGKDD Explor. Newsl., 9(2):80-83, Dec. 2007.

[192] L. Tang, Y. Jiang, L. Li, and T. Li. Ensemble contextual bandits for personalized recommendation. In Proc. of the 8th ACM Conference on Recommender Systems, RecSys '14, pages 73-80. ACM, 2014.

[193] T. Tang and P. Winoto. I should not recommend it to you even if you will like it: the ethics of recommender systems. New Review of Hypermedia and Multimedia, 22(1-2):111-138, 2016.

[194] H. Tavani. Ethics and technology: Controversies, questions and strategies for ethical computing. Wiley, 2015.

[195] N. Tintarev and J. Masthoff. Designing and evaluating explanations for recommender systems. In F. Ricci, L. Rokach, B. Shapira, and P. B. Kantor, editors, Recommender Systems Handbook, pages 479-510. Springer US, 2011.

[196] B. Twardowski. Modelling contextual information in sessionaware recommender systems with neural networks. In Proc. of the 10th ACM Conference on Recommender Systems, RecSys '16, pages 273-276. ACM, 2016.

[197] T. Uchiya, A. Nakamura, and M. Kudo. Algorithms for adversarial bandit problems with multiple plays. In Proc. of the 21st 
International Conference on Algorithmic Learning Theory, ALT'10, pages 375-389. Springer-Verlag, 2010.

[198] L. Ungar, D. Foster, E. Andre, S. Wars, F. S. Wars, D. S. Wars, and J. H. Whispers. Clustering methods for collaborative filtering. In Proc. of the Workshop on Recommendation Systems. AAAI Press, 1998.

[199] V. K. Vaishnavi and W. Kuechler, Jr. Design science research methods and patterns: Innovating information and communication technology. Auerbach Publications, 1st edition, 2007.

[200] J. Valentino-DeVries, J. Singer-Vine, and A. Soltani. Websites vary prices, deals based on users' information. Online: www.wsj.com/articles/ SB10001424127887323777204578189391813881534/, December 2012. Accessed: 2016-05-02.

[201] S. B. van der Zon. Predictive performance and discrimination in unbalanced classification. Master's thesis, Eindhoven University of Technology, 2016.

[202] S. Verwer and T. Calders. Introducing positive discrimination in predictive models. In B. Custers, T. Calders, B. Schermer, and T. Zarsky, editors, Discrimination and Privacy in the Information Society, chapter 14, pages 255-270. Springer Berlin Heidelberg, 2013.

[203] E. G. Vozalis and K. G. Margaritis. Recommender systems: An experimental comparison of two filtering algorithms. In Proc. of the Ninth Panhellenic Conference in Informatics, 2003.

[204] W. Wallach and C. Allen. Moral machines: teaching robots right from wrong. Ethics and Information Technology, 12(4):359-361, Dec 2010.

[205] J. Wang and Y. Zhang. Utilizing marginal net utility for recommendation in e-commerce. In Proc. of the 34th International ACM SIGIR Conference on Research and Development in Information Retrieval, SIGIR '11, pages 1003-1012. ACM, 2011.

[206] X. Wang, Y. Wang, D. Hsu, and Y. Wang. Exploration in interactive personalized music recommendation: A reinforcement learning approach. ACM Trans. Multimedia Comput. Commun. Appl., 11(1):7:1-7:22, 2014. 
[207] Y. Wang and A. Kobsa. Respecting users' individual privacy constraints in web personalization. In C. Conati, K. McCoy, and G. Paliouras, editors, User Modeling 2007: 11th International Conference, pages 157-166. 2007.

[208] C. Wohlin. Guidelines for snowballing in systematic literature studies and a replication in software engineering. In Proc. of the 18th International Conference on Evaluation and Assessment in Software Engineering, EASE '14, pages 38:1-38:10. ACM, 2014.

[209] D. H. Wolpert. Original contribution: Stacked generalization. Neural Netw., 5(2):241-259, Feb. 1992.

[210] C. Wu, M. Yan, and L. Si. Session-aware information embedding for e-commerce product recommendation. CoRR, abs/1707.05955, 2017.

[211] M. Wu. Collaborative filtering via ensembles of matrix factorization. In KDDCup 2007, pages 43-47, 2007.

[212] Y. Xu, T. Ma, M. Tang, and T. Wei. A survey of privacy preserving data publishing using generalization and suppression. Applied Mathematics \& Information Sciences, 8:1103-1116, May 2014.

[213] M. J. Zaki. Scalable algorithms for association mining. IEEE Transactions on Knowledge and Data Engineering, 12(3):372390, May 2000.

[214] J. Zhan, C.-L. Hsieh, I.-C. Wang, T.-S. Hsu, C.-J. Liau, and D.-W. Wang. Privacy-preserving collaborative recommender systems. Trans. Sys. Man Cyber Part C, 40(4):472-476, July 2010.

[215] G. Zhao, M. L. Lee, W. Hsu, and W. Chen. Increasing temporal diversity with purchase intervals. In Proc. of the 35th International ACM SIGIR Conference on Research and Development in Information Retrieval, SIGIR '12, pages 165-174. ACM, 2012.

[216] G. Zhao, M. L. Lee, and H. Wynne. Utilizing purchase intervals in latent clusters for product recommendation. In Proc. of the 8th Workshop on Social Network Mining and Analysis, SNAKDD'14, pages 4:1-4:9. ACM, 2014.

[217] Q. Zhao, Y. Zhang, D. Friedman, and F. Tan. E-commerce recommendation with personalized promotion. In Proc. of the 9th ACM Conference on Recommender Systems, RecSys '15, pages 219-226. ACM, 2015. 
[218] X. Zhao, W. Zhang, and J. Wang. Interactive collaborative filtering. In Proc. of the 22nd ACM international conference on Conference on Information and Knowledge Management, CIKM '13, pages 1411-1420. ACM, 2013. 

ISBN 978-9I-7I04-900-I (print)

ISBN 978-9I-7IO4-90I-8 (pdf)

MALMÖ UNIVERSITY 20506 MALMÖ, SWEDEN WWW.MAU.SE 